\title{
High-temperature asymptotics of supersymmetric partition functions
}

\author{
Arash Arabi Ardehali \\ Michigan Center for Theoretical Physics, Randall Laboratory of Physics, \\ The University of Michigan, Ann Arbor, MI 48109-1040, U.S.A. \\ E-mail: ardehali@umich.edu
}

ABSTRACT: We study the supersymmetric partition function of $4 \mathrm{~d}$ supersymmetric gauge theories with a U(1) R-symmetry on Euclidean $S^{3} \times S_{\beta}^{1}$, with $S^{3}$ the unit-radius squashed three-sphere, and $\beta$ the circumference of the circle. For superconformal theories, this partition function coincides (up to a Casimir energy factor) with the $4 \mathrm{~d}$ superconformal index.

The partition function can be computed exactly using the supersymmetric localization of the gauge theory path-integral. It takes the form of an elliptic hypergeometric integral, which may be viewed as a matrix-integral over the moduli space of the holonomies of the gauge fields around $S_{\beta}^{1}$. At high temperatures $(\beta \rightarrow 0$, corresponding to the hyperbolic limit of the elliptic hypergeometric integral) we obtain from the matrix-integral a quantum effective potential for the holonomies. The effective potential is proportional to the temperature. Therefore the high-temperature limit further localizes the matrix-integral to the locus of the minima of the potential. If the effective potential is positive semi-definite, the leading high-temperature asymptotics of the partition function is given by the formula of Di Pietro and Komargodski, and the subleading asymptotics is connected to the Coulomb branch dynamics on $R^{3} \times S^{1}$. In theories where the effective potential is not positive semi-definite, the Di Pietro-Komargodski formula needs to be modified. In particular, this modification occurs in the SU(2) theory of Intriligator-Seiberg-Shenker, and the $\mathrm{SO}(N)$ theory of Brodie-Cho-Intriligator, both believed to exhibit "misleading" anomaly matchings, and both believed to yield interacting superconformal field theories with $c<a$.

Two new simple tests for dualities between $4 \mathrm{~d}$ supersymmetric gauge theories emerge as byproducts of our analysis.

KeYwords: Matrix Models, Supersymmetric gauge theory, Supersymmetry and Duality

ARXIV EPRINT: 1512.03376 


\section{Contents}

1 Introduction $\quad 2$

$\begin{array}{lll}1.1 & \text { Notation and terminology } & 6\end{array}$

$\begin{array}{lll}1.2 & \text { Relation to previous work } & 7\end{array}$

2 Mathematical background $\quad 9$

2.1 Useful special functions $\quad 9$

$\begin{array}{lll}2.2 & \text { Some asymptotic analysis } & 10\end{array}$

$\begin{array}{lll}2.3 & \text { Generalized triangle inequalities } & 13\end{array}$

3 Asymptotics of the SUSY partition function $\quad 14$

$\begin{array}{lll}3.1 & Z_{S^{3}} \text { finite } & 22\end{array}$

3.1.1 $A_{k}$ SQCD theories with $N_{f}>\frac{2 N}{k+1} \quad 24$

$\begin{array}{ll}\text { 3.1.2 The magnetic Pouliot theory with } N_{f}=7 & 26\end{array}$

$3.2 Z_{S^{3}}$ power-law divergent (or: the effect of an unlifted Coulomb branch) 27

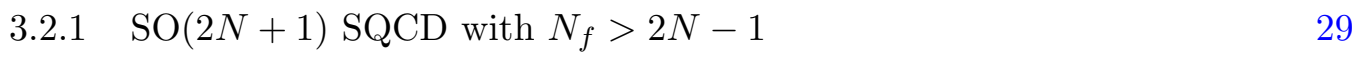

$\begin{array}{lll}3.2 .2 & \mathrm{SU}(N) \mathcal{N}=4 \mathrm{SYM} & 32\end{array}$

3.2.3 The $\mathbb{Z}_{2}$ orbifold theory 33

3.2.4 The $\mathbb{Z}_{3}$ orbifold theory 34

$3.3 Z_{S^{3}}$ exponentially divergent (or: the curious case of the SCFTs with $c<a$ ) 35

3.3.1 The SU(2) ISS model 36

3.3.2 The $\mathrm{SO}(2 N+1)$ BCI model with $1<N<5 \quad 38$

4 Asymptotics of the $\mathcal{N}=2$ partition function $\quad 40$

4.1 Asymptotics of the Schur partition function and the Schur index 42

4.1.1 The Schur partition function of $\operatorname{SU}(N) \mathcal{N}=4 \mathrm{SYM}$

4.2 The example of the non-Lagrangian $E_{6}$ SCFT 44

5 Discussion $\quad 46$

5.1 Open problems 48

5.2 Two new simple tests of supersymmetric dualities 48

5.3 Holography and the asymptotics of $4 \mathrm{~d}$ superconformal indices 49

5.4 Crossed channel: quantum Coulomb branch dynamics on $R^{3} \times S^{1} \quad 50$

A Derivation of the elliptic gamma function estimates $\quad 52$

B Asymptotics of the Schur partition function of the $\mathcal{N}=4$ theory $\quad 53$ 


\section{Introduction}

Knowledge of the high-temperature asymptotics of the elliptic genera of $2 \mathrm{~d}$ superconformal field theories (SCFTs) has allowed micro-state counting of certain supersymmetric Black Holes [1-3]. In this work we study the high-temperature asymptotics of the $4 \mathrm{~d}$ ana$\log$ of the elliptic genus. This is the supersymmetric (SUSY) partition function $Z^{\mathrm{SUSY}}(b, \beta)$, defined by the path-integral of the theory on Euclidean $S_{b}^{3} \times S_{\beta}^{1}$, with $\beta$ the circumference of the circle, and $S_{b}^{3}$ the unit three-sphere with squashing parameter $b$; the round threesphere corresponds to $b=1$, and we assume $b$ to be a positive real number throughout this paper. The superscript SUSY is added to emphasize that $i$ ) the path-integral is computed with periodic boundary conditions around the circle, $i i$ ) the Lagrangian used for pathintegration is made compatible with supersymmetry on $S_{b}^{3} \times S_{\beta}^{1}$, and iii) a background $\mathrm{U}(1)_{R}$ gauge field is turned on along $S_{\beta}^{1}$ in order to make the supercharges independent of the "time" coordinate parameterizing the circle (see $[4,5]$ ). In analogy with thermal quantum physics we refer to $\beta$ as the "inverse temperature" - even though our fermions do not have thermal (i.e. anti-periodic) boundary condition around $S_{\beta}^{1}$.

Because of the condition iii above, for $Z^{\mathrm{SUSY}}$ to be well-defined we need a $\mathrm{U}(1)_{R}$ symmetry in the theory, whose existence we take for granted below; the presence of the greater superconformal symmetry is not necessary. For superconformal theories, however, $Z^{\mathrm{SUSY}}$ becomes more significant, and coincides (up to a Casimir energy factor) with the $4 \mathrm{~d}$ superconformal index of $[4,6]$, which counts the protected operators in the theory.

Unlike the $2 \mathrm{~d}$ elliptic genera, the 4d SUSY partition functions - or alternatively the 4d superconformal indices - of holographic SCFTs do not seem to encode Black Hole physics [6], but they may aid the microscopic counting of supersymmetric Giant Gravitons [7].

The asymptotics of the elliptic genera of $2 \mathrm{~d}$ SCFTs are well-known, thanks to their simple modular properties (see for instance [3]). In four dimensions, on the other hand, analogous general results for the asymptotics of $Z^{\text {SUSY }}$ have only begun to appear recently. Di Pietro and Komargodski have combined ideas from supersymmetry and hydrodynamics to argue [8] that the SUSY partition functions of 4d Lagrangian theories exhibit the following universal behavior at the leading order:

$$
\ln Z^{\mathrm{SUSY}}(b, \beta) \approx-\frac{\pi^{2}}{3 \beta}\left(\frac{b+b^{-1}}{2}\right) \operatorname{Tr} R, \quad(\text { as } \beta \rightarrow 0)
$$

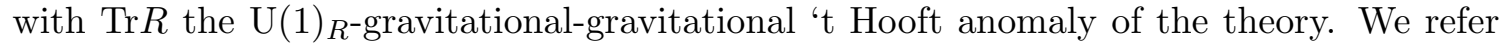
to the above relation as the Di Pietro-Komargodski formula.

The supporting arguments for (1.1) are, however, somewhat indirect, and contain some intuitive elements that we would like to scrutinize by a more direct analysis.

In $[8,9]$ the relation (1.1) was directly verified for free chiral and $\mathrm{U}(1)$ vector multiplets. In the present work we extend the analysis to interacting supersymmetric gauge theories with a semi-simple gauge group. [Our approach enables us to study also the nonLagrangian $E_{6}$ SCFT; see subsection 4.2.] The SUSY partition function of such theories 
can be computed exactly using the supersymmetric localization [10]. We write this as

$$
Z^{\mathrm{SUSY}}(b, \beta):=\int e^{-S} \mathcal{D} \phi \mathcal{D} A \stackrel{\text { SUSY localization }}{\longrightarrow} \int \mathrm{d}^{r_{G}} x f\left(x_{1}, \ldots, x_{r_{G}} ; b, \beta\right),
$$

with $r_{G}$ the rank of the gauge group, $f$ some complicated function of its arguments, and the integral on the (far) right over the range $-1 / 2 \leq x_{i} \leq 1 / 2$. The path-integral of the theory on $S_{b}^{3} \times S_{\beta}^{1}$ is displayed schematically as $\int e^{-S} \mathcal{D} \phi \mathcal{D} A$, with $\phi$ and $A$ representing the matter fields and the gauge fields respectively.

The $x_{i}$ in (1.2) parameterize the unit hypercube in the Cartan subalgebra of the gauge group; we denote this hypercube by $\mathfrak{h}_{c l}$. The exponential function $z_{i}=e^{2 \pi i x_{i}}$ maps $\mathfrak{h}_{c l}$ to the moduli space of the eigenvalues of the holonomy matrix $P \exp \left(i \oint_{S_{\beta}^{1}} A_{0}\right)$, with $A_{0}$ the component of $A$ along $S_{\beta}^{1}$. The (matrix-) integral on the (far) right of (1.2) is thus over the "classical" moduli space of the holonomies around the circle; hence the subscript $c l$ in $\mathfrak{h}_{c l}$.

The matrix-integrals appearing in $Z^{\mathrm{SUSY}}(b, \beta)$ are known in the mathematics literature as elliptic hypergeometric integrals (EHIs) $[11,12]$. The high-temperature limit corresponds to the hyperbolic limit of the EHIs. This limit can be rigorously analyzed with the machinery that Rains has developed in [13]. Following Rains's approach, we find that at high temperatures the integrand of the matrix-integral in (1.2) simplifies as ${ }^{1}$

$$
f\left(x_{1}, \ldots, x_{r_{G}} ; b, \beta\right) \stackrel{\beta \rightarrow 0}{\longrightarrow} \exp \left[-\left(\mathcal{E}_{0}^{D K}(b, \beta)+V^{\mathrm{eff}}\left(x_{1}, \ldots, x_{r_{G}} ; b, \beta\right)\right)\right],
$$

where

$$
\mathcal{E}_{0}^{D K}(b, \beta)=\frac{\pi^{2}}{3 \beta}\left(\frac{b+b^{-1}}{2}\right) \operatorname{Tr} R,
$$

and $V^{\text {eff }}$ is a real, continuous, piecewise linear function of the $x_{i}$ (examples can be found in figures 2,6 , and 8 below). We interpret $V^{\text {eff }}$ as a quantum effective potential for the interaction of the holonomies. This is of course not a low-energy effective potential from the perspective of the three-sphere; rather, it is loosely a "high-energy effective potential", as it governs the high-temperature behavior of the SUSY partition function. In section 5 we will discuss the extent to which an alternative viewpoint (roughly speaking, from a crossed channel) allows considering $V^{\text {eff }}$ as a conventional (low-energy) quantum effective potential.

We find that $V^{\mathrm{eff}}$ is inversely proportional to $\beta$. Therefore the high-temperature limit further localizes the matrix-integral to the locus of the minima of $V^{\text {eff }}$. This locus is a subspace of $\mathfrak{h}_{c l}$ that we denote by $\mathfrak{h}_{q u}$. We can thus combine (1.2) and (1.3) to write

$$
Z^{\mathrm{SUSY}}(b, \beta) \stackrel{\beta \rightarrow 0}{\longrightarrow} \int_{\mathfrak{h}_{q u}} e^{-\left(\mathcal{E}_{0}^{D K}(b, \beta)+V^{\mathrm{eff}}\left(x_{1}, \ldots, x_{r_{G}} ; b, \beta\right)\right)} \approx e^{-\left(\mathcal{E}_{0}^{D K}(b, \beta)+V_{\min }^{\mathrm{eff}}(b, \beta)\right)},
$$

with $V_{\min }^{\text {eff }}$ the minimum of $V^{\text {eff }}$ over $\mathfrak{h}_{c l}$ - or alternatively the value of $V^{\text {eff }}$ on $\mathfrak{h}_{q u}$.

A similar "high-temperature localization" of path-integrals has long been known to occur in non-supersymmetric pure gauge theories on Euclidean $R^{3} \times S^{1}[14,15]$. In our

\footnotetext{
${ }^{1}$ In the present section we assume the theories under study have non-chiral matter content. Otherwise, some of the following expressions need to be slightly modified. We will comment on the case of theories with chiral matter content in section 3 .
} 
case the problem is more under control for two reasons. Firstly, since the spatial manifold that our theories live on is compact, our path-integrals are finite and do not need IR regularization. Secondly, thanks to the supersymmetric localization, we have the luxury of having at our disposal the exact partition function of the interacting gauge theory, which we can then study using standard methods of asymptotic analysis. In the nonsupersymmetric cases of $[14,15]$, on the other hand, the high-temperature limit is employed to seek approximate results.

It turns out that $V^{\text {eff }}$ vanishes at the origin of $\mathfrak{h}_{c l}$ (corresponding to $x_{i}=0$ ). Therefore its minimum $V_{\min }^{\text {eff }}$ is guaranteed to be $\leq 0$. In a large set of examples we find that $V_{\min }^{\text {eff }}=0$, and consequently recover the Di Pietro-Komargodski formula (1.1) from (1.5).

For some interacting theories, however, we find that $V_{\text {min }}^{\text {eff }}<0$ (see figures 8 and 9 ). In such cases (1.5) implies that the formula (1.1) receives a modification:

$$
\ln Z^{\mathrm{SUSY}}(b, \beta) \approx-\frac{\pi^{2}}{3 \beta}\left(\frac{b+b^{-1}}{2}\right) \operatorname{Tr} R-V_{\min }^{\mathrm{eff}}(b, \beta) \quad(\text { as } \beta \rightarrow 0) .
$$

We are aware of only two examples where this modification occurs. One is the $\mathrm{SU}(2)$ Intriligator-Seiberg-Shenker theory [16], and the other is the $\mathrm{SO}(N)$ theory of Brodie-ChoIntriligator [17]. Both of these are believed to exhibit "misleading" anomaly matchings, and both have $\operatorname{Tr} R>0$ (or alternatively, $c<a$ for the putative IR fixed points). Interestingly, we find that in both cases the correction term coming from $V_{\min }^{\text {eff }}<0$ makes the r.h.s. of (1.6) positive.

A possible explanation for why the result of Di Pietro and Komargodski does not apply when $V_{\mathrm{min}}^{\text {eff }}<0$ is as follows. As stated in subsection 4.3 of [8], an assumption made in that work is that the $S_{b}^{3}$ partition function $Z_{S^{3}}(b)$ of the 4 d theory reduced on $S_{\beta}^{1}$ does not diverge. Indeed, in all the theories with finite $Z_{S^{3}}$ that we have studied, $V_{\min }^{\mathrm{eff}}$ vanishes and consequently (1.1) is satisfied. [We have not been able to show that the finiteness of $Z_{S^{3}}$ always implies $V_{\min }^{\text {eff }}=0$, although we suspect that is the case; we will comment on this point further in section 5.]

There exist theories with $V_{\mathrm{min}}^{\mathrm{eff}}=0$, but in which $V^{\mathrm{eff}}$ has flat directions and the locus of the high-temperature localization is extended: $\operatorname{dimh} \mathfrak{h}_{q u}>0$. In such cases $Z_{S^{3}}$ diverges, and therefore the arguments in [8] are not on solid footing. The matrix-integral that computes $Z_{S^{3}}$ (via 3d supersymmetric localization [18-20]) must then be regularized with a cut-off. Introducing a cut-off $\Lambda$, we argue in section 3 that upon taking $\Lambda \rightarrow \infty$, the $S_{b}^{3}$ partition function diverges in these cases as $\Lambda^{\text {dimh }_{q u}}$. The power-law divergences in $Z_{S^{3}}$ were interpreted in [8] as coming from the "unlifted Coulomb branch" of the reduced theory on $S_{b}^{3}$. Di Pietro and Komargodski presented intuitive arguments suggesting that for theories with such unlifted Coulomb branches, the relation (1.1) remains valid at the leading order, but there will be subleading corrections to it of the form $\ln (1 / \beta)$. We will show in section 3 that, when $V_{\text {min }}^{\text {eff }}=0$, the Di Pietro-Komargodski formula for the leading asymptotics indeed remains valid, and the subleading correction to it is of the form $\operatorname{dimh} \mathfrak{h}_{q u} \cdot \ln (1 / \beta)$. If one interprets $\mathfrak{h}_{q u}$ as the "quantum Coulomb branch" of the reduced theory on $S_{b}^{3}$, this subleading correction is in accord with the prescription of Di Pietro and Komargodski. Furthermore, in section 5 we will argue intuitively that, when $V_{\min }^{\text {eff }}=0$, 
the space $\mathfrak{h}_{q u}$ should resemble the unlifted (or quantum) Coulomb branch of the $3 \mathrm{~d}$ theory obtained by reducing the gauge theory on the circle of $R^{3} \times S^{1}$.

In the examples where $V_{\text {min }}^{\text {eff }}<0$, however, we find that $Z_{S^{3}}$ diverges exponentially in $\Lambda$, as $\Lambda \rightarrow \infty$. This severe divergence seems to undermine the - three-dimensional assumption of Di Pietro and Komargodski. As a result, the formula (1.1) no longer holds, and the correct asymptotics of the SUSY partition function is given by (1.6).

A refinement of the SUSY partition function is available for Lagrangian $\mathcal{N}=2$ SCFTs. These have extended R-symmetry group $\mathrm{SU}(2)_{R_{\mathcal{N}=2}} \times \mathrm{U}(1)_{r_{\mathcal{N}=2}}$. We can then consider the $\mathcal{N}=2$ partition function $Z^{\mathcal{N}=2}\left(b, \beta, m_{v}\right)$, where $m_{v}$ is a background $\mathrm{U}(1)_{v}$ gauge field along $S_{\beta}^{1}$, that couples to a specific linear combination of $\mathrm{U}(1)_{r_{\mathcal{N}=2}}$ and the Cartan of $\mathrm{SU}(2)_{R_{\mathcal{N}=2}}$. We will analyze the asymptotics of this partition function in section 4 . The Schur limit [21] of $Z^{\mathcal{N}=2}\left(b, \beta, m_{v}\right)$, defined by setting $b=1$ and $m_{v}=i / 3$, has been the subject of much recent work. We will show in section 4 that the high-temperature asymptotics of the Schur partition function is given by

$$
\ln Z^{\operatorname{Schur}}(\beta) \approx-\frac{\pi^{2}}{2 \beta} \operatorname{Tr} R-\frac{3}{2} V_{\min }^{\mathrm{eff}}(b=1, \beta) \quad(\text { as } \beta \rightarrow 0) .
$$

In particular, when $V_{\mathrm{min}}^{\mathrm{eff}}=0$ - which is when the Di Pietro-Komargodski formula for $Z^{\mathrm{SUSY}}(b, \beta)$ applies - we find

$$
\ln Z^{\operatorname{Schur}}(\beta) \approx-\frac{\pi^{2}}{2 \beta} \operatorname{Tr} R \quad\left(\text { as } \beta \rightarrow 0, \text { when } V_{\min }^{\text {eff }}=0\right) .
$$

This relation was recently observed by Buican and Nishinaka to hold in a large set of Lagrangian and non-Lagrangian examples [22].

Dual gauge theories must have identical partition functions. Comparison of the SUSY partition functions of supersymmetric gauge theories with a U(1) R-symmetry provides one of the strongest tests of any proposed duality between such theories [11, 12]. The full comparison of the matrix-integrals computing such partition functions is, however, extremely challenging, except for the few cases (corresponding to various SQCD-type theories [11, 12, 23]) already established in the mathematics literature (e.g. [24]). Rather, known dualities are frequently used to conjecture new identities between multi-variable matrix-integrals of elliptic hypergeometric type [11, 12, 23, 25].

We propose comparison of the high-temperature asymptotics of the SUSY partition functions. This comparison provides two new simple tests for dualities between SUSY gauge theories with a U(1) R-symmetry. The first test is the comparison of $V_{\min }^{\mathrm{eff}}$, which according to (1.6) determines the leading high-temperature asymptotics of the SUSY partition functions. The second test is the comparison of the dimension of the locus of minima of $V^{\text {eff }}$ - i.e. $\operatorname{dimh} \mathfrak{h}_{q u}$; this is an integer which, as we briefly mentioned above, determines the subleading $\ln (1 / \beta)$ term in the high-temperature asymptotics of $\ln Z^{\mathrm{SUSY}}$. These two high-temperature tests are independent of 't Hooft anomaly matchings (which in turn can be thought of as arising from comparison of the low-temperature asymptotics of an equivariant generalization of $\left.Z^{\mathrm{SUSY}}[26]\right)$. They may thus help to diagnose situations with misleading anomaly matchings. See subsection 5.2 for a few concrete applications of these two duality tests. 
The rest of this paper is organized as follows. In the remaining of the present section we first summarize our notation and terminology, and then proceed to mention the relation of our findings to previous work.

In section 2 we present the mathematical background required for the quantitative analysis in the body of the paper. The main result of section 2 is the uniform estimate (2.11) for the high-temperature asymptotics of the elliptic gamma function.

Section 3 contains our main findings. There we show the high-temperature localization of the SUSY partition function $Z^{\mathrm{SUSY}}(b, \beta)$, obtain the effective potential $V^{\text {eff }}$ that determines the locus of the high-temperature localization, establish the validity of the Di Pietro-Komargodski formula (1.1) when $V^{\text {eff }}$ is positive semi-definite, and demonstrate its modified version (1.6) for theories with $V_{\text {min }}^{\text {eff }}<0$. Section 3 is the lengthiest section of this paper, partly because it includes several examples that are analyzed quite explicitly.

In section 4 we analyze the high-temperature asymptotics of the $\mathcal{N}=2$ partition function $Z^{\mathcal{N}=2}\left(b, \beta, m_{v}\right)$, and establish the formula (1.7) for the asymptotics of its Schur limit. Section 4 includes also the high-temperature analysis of the superconformal index of the $E_{6} \mathrm{SCFT}$, which is the only non-Lagrangian theory studied in this paper.

Our concluding remarks are made in section 5 , and the appendices contain some technical details that are not essential for following the discussion in the main text.

\subsection{Notation and terminology}

Partition functions and indices. The SUSY partition function $Z^{\mathrm{SUSY}}(b, \beta)$ is the path-integral of the $4 \mathrm{~d}$ Lagrangian supersymmetric R-symmetric theory on Euclidean $S_{b}^{3} \times$ $S_{1}^{\beta}$, in presence of a specific (as in $[4,5]$ ) background $\mathrm{U}(1)_{R}$ gauge field along $S_{1}^{\beta}$, and with periodic boundary conditions around the circle. This is the object computed by supersymmetric localization in [10,27], and their result (with a minor correction of a regularization procedure, as explained in $[9,28]$ ) is our starting point. For superconformal theories, $Z^{\mathrm{SUSY}}(b, \beta)$ coincides, up to a Casimir energy factor (see eq. (3.1) below), with the superconformal index $\mathcal{I}(b, \beta)$, which we sometimes refer to as the Romelsberger index, or simply as the index. More commonly, the index is written as a function of $p, q$, which are related to $b, \beta$ via $p=e^{-\beta b}, q=e^{-\beta b^{-1}}$. Alternatively, we can express the partition function or the index, in terms of the complex structure moduli $\sigma, \tau$ of the space $S_{b}^{3} \times S_{1}^{\beta}$; these are related to $p, q$ via $p=e^{2 \pi i \sigma}, q=e^{2 \pi i \tau}$. We always assume $b, \beta$ to be positive real numbers, and thus $\sigma, \tau$ to be pure imaginary in the upper half plane; $p, q$ are then real numbers in ]0,1[. The high-temperature limit corresponds to $\beta \rightarrow 0$ with $b$ fixed.

For non-conformal supersymmetric gauge theories with well-defined $Z^{\mathrm{SUSY}}(b, \beta)$, we take eq. (3.1) below as the definition of the Romelsberger index (or the index) $\mathcal{I}(b, \beta)$. This way we avoid the awkward use of the term "superconformal index" for nonconformal theories.

A further background gauge field $m_{v}$, which we take to be pure imaginary and in the upper half plane, can serve to refine the partition functions of $\mathcal{N}=2$ SCFTs with R-symmetry group $\mathrm{SU}(2)_{R_{\mathcal{N}=2}} \times \mathrm{U}(1)_{r_{\mathcal{N}=2}}$. The charge $Q_{v}$ that $m_{v}$ couples to is

$$
Q_{v}=-\left(r_{\mathcal{N}=2}+R_{\mathcal{N}=2}\right) .
$$


We denote the resulting partition function by $Z^{\mathcal{N}=2}\left(b, \beta, m_{v}\right)$, and refer to it as the $\mathcal{N}=\mathbf{2}$ partition function. This partition function coincides, up to a Casimir energy factor (see eq. (4.4) below), with the $\mathcal{N}=\mathbf{2}$ superconformal index $\mathcal{I}\left(b, \beta, m_{v}\right)$, which we frequently refer to as the $\mathcal{N}=\mathbf{2}$ index. The high-temperature limit corresponds to $\beta \rightarrow 0$ with $b, m_{v}$ fixed.

Special functions. The $q$-Pochhammer symbol, often written in the mathematics literature as $(a ; q)_{\infty}$, will be denoted below by $(a ; q)$, and will be called the Pochhammer symbol.

The elliptic gamma function, commonly written as $\Gamma_{e}(z ; p, q)$, will be denoted below by $\Gamma(z ; p, q)$. We sometimes write $\Gamma(z ; p, q)$ as $\Gamma(x ; \sigma, \tau)$, or simply as $\Gamma(z)$. Also, the arguments of elliptic gamma functions are frequently written with "ambiguous" signs (as in $\Gamma( \pm x ; \sigma, \tau))$; by that one means a multiplication of several gamma functions each with a "possible" sign of the argument (as in $\Gamma(+x ; \sigma, \tau) \times \Gamma(-x ; \sigma, \tau))$. Similarly $\Gamma\left(z^{ \pm 1}\right):=$ $\Gamma(z ; p, q) \times \Gamma\left(z^{-1} ; p, q\right)$.

The hyperbolic gamma function will be denoted by the standard $\Gamma_{h}\left(x ; \omega_{1}, \omega_{2}\right)$, with $\omega_{1}=i b$ and $\omega_{2}=i b^{-1}$. For convenience, we will frequently write $\Gamma_{h}(x)$ instead of $\Gamma_{h}\left(x ; \omega_{1}, \omega_{2}\right)$, and $\Gamma_{h}(x \pm y)$ instead of $\Gamma_{h}(x+y) \Gamma_{h}(x-y)$.

Asymptotic analysis. When writing asymptotic relations, we use the symbol $\sim$ to indicate all-orders asymptotic equalities. For example, we write $f(\beta) \sim g(\beta)$, if the small$\beta$ asymptotic expansions of $f(\beta)$ and $g(\beta)$ coincide to all orders in $\beta$. This notation is standard, and appears, for instance, in [29].

We will also use the non-standard notation $f(\beta) \simeq g(\beta)$, whenever $\ln f(\beta) \sim \ln g(\beta)$.

Finally, we use the symbol $\approx$ to indicate "approximate asymptotic equality". We will not make this statement more precise, and instead explicitly mention the error involved whenever using $\approx$ below.

Convex polytopes. By a $j$-face we mean an element of dimension $j$ in a convex polytope. We define the unique $d$-face of a $d$-dimensional polytope to be the polytope itself.

We call a $d$-dimensional polytope a prismatoid if all its vertices (i.e. 0 -faces) lie in either of two parallel codimension one hyperplanes. A prismatoid with only one vertex in one of the two hyperplanes will be referred to as a pyramid.

\subsection{Relation to previous work}

Our discussion of the high-temperature asymptotics of the SUSY partition function of Lagrangian gauge theories relies heavily on the machinery developed by Rains [13]. In fact Rains's results are immediately applicable to $\mathrm{SU}(N)$ and $\mathrm{Sp}(N)$ SQCD-type theories, and yield asymptotics of the form (1.1).

The fact that Rains's method gives the leading high-temperature asymptotics of the Romelsberger index $\mathcal{I}(b, \beta)$ in accord with the formula (1.1) was identified and pointed out for $\mathrm{SU}(N)$ and $\mathrm{Sp}(N) \mathrm{SQCD}$-type theories in [30,31] and [32]. The relation (3.1), which was obtained later in $[9,28]$, would then imply the formula (1.1) for $Z^{\mathrm{SUSY}}$. (Other pioneering works on the high-temperature limit of the $4 \mathrm{~d}$ superconformal index include [33, 34], which 
clarified the relation between the $4 \mathrm{~d}$ index and the $S^{3}$ partition function, but did not address the Cardy-like asymptotics of the index.)

An argument for the general validity of the formula (1.1) appeared first in the work of Di Pietro and Komargodski [8], who used methods completely different from those of Rains. Importantly, Di Pietro and Komargodski improved the qualitative understanding of the role of unlifted Coulomb branches in the high-temperature asymptotics of the index [35, 36], to a quantitative discussion and argued that such unlifted Coulomb branches would only introduce subleading logarithmic corrections to the formula (1.1), but would not modify the leading behavior.

In the present paper we show that Rains's rigorous approach can be adapted, with minor modifications, for analyzing any SUSY partition function (or Romelsberger index) given as an elliptic hypergeometric integral. We are thus able to find the conditions under which the Di Pietro-Komargodski formula applies. In particular, we find that the formula (1.1) does not apply in certain interacting SCFTs with $c<a$.

In $[37,38]$ certain results from holography were derived, over which we do not present any improvement here. However, the holographic - large- $N$ - results were extrapolated there to conjecture prescriptions for extracting the central charges of any finite- $N$ SCFT from its superconformal index. In [9] it was shown that those prescriptions are equivalent to the statement that there is no $O(\beta)$ term in the high-temperature asymptotics of $\ln Z^{\mathrm{SUSY}}(\beta)$. In the present paper we almost establish that this statement is correct whenever $\operatorname{dimh}_{q}=0$ (see the comments below (3.82)). On the other hand, we find a counterexample which has $\operatorname{dimh}_{q}=1$ : for the $\mathrm{SO}(3) \mathrm{SQCD}$ with two flavors, $\ln Z^{\mathrm{SUSY}}(\beta)$ does have an $O(\beta)$ term in its high-temperature expansion (see (3.64) below). Our results thus indicate that the finite- $N$ conjectures of $[37,38]$ are not necessarily true if $\operatorname{dimh} \mathfrak{h}_{q u}>0$.

In [9] the SUSY partition function of free U(1) vector and free chiral multiplets were studied in the high-temperature limit. The corresponding expressions were then conjectured to be true for all SCFTs (with finite $N$ ). Here we rule out that possibility, although we find that, except for the $\ln (1 / \beta)$ term in the asymptotics of $\ln Z^{\mathrm{SUSY}}(\beta)$ conjectured in that work, the conjecture in [9] is correct for theories whose $V^{\text {eff }}$ has a unique minimum at the origin of $\mathfrak{h}_{c l}$.

We have organized our discussion in section 3 according to the degree of divergence of $Z_{S^{3}}$. For certain $3 \mathrm{~d} \mathcal{N}=4$ theories, the criteria for finiteness of $Z_{S^{3}}$ have already been analyzed in three-dimensional terms [39] (see also [40, 41] for related discussions in the context of a particular $3 \mathrm{~d} \mathcal{N}=2$ model). Our perspective on this problem is a bit different, as we consider $3 \mathrm{~d} \mathcal{N}=2$ theories obtained from dimensional reduction of $4 \mathrm{~d}$ $\mathcal{N}=1$ gauge theories whose index we would like to study.

Finally, Buican and Nishinaka [22] recently noted a Cardy-like behavior in the Schur index of a variety of Lagrangian and non-Lagrangian $\mathcal{N}=2$ theories. We show in the present paper that for all Lagrangian theories with a semi-simple gauge group the Cardylike behavior noted in [22] is valid in theories where the Di Pietro-Komargodski formula for the SUSY partition function is satisfied - i.e. when $V^{\text {eff }}$ is positive semi-definite. 


\section{Mathematical background}

In subsection 2.1 below, we define the Pochhammer symbol, the elliptic gamma function, and the hyperbolic gamma function.

In subsection 2.2 we review the asymptotic estimates of the special functions discussed in subsection 2.1. These estimates form the mathematical basis of our high-temperature analysis of SUSY partition functions. The only new estimate, and the main result of the present section, is the relation (2.11) for the asymptotics of the elliptic gamma function. All the other estimates for the elliptic and hyperbolic gamma functions have appeared (sometimes in slightly different forms) already in the work of Rains [13]; we only present them in a way more suited for the physical application. Even the estimate (2.11) is only a minor modification of the results in Proposition 2.12 and Corollary 3.1 of [13].

The important estimates are the asymptotics of the Pochhammer symbol in eq. (2.9), the "leading estimate" (2.11) and the "central estimate" (2.16) for the elliptic gamma function, and the asymptotics of the hyperbolic gamma function in eq. (2.19).

Subsection 2.3 contains generalized triangle inequalities due to Rains [13], that we will need in the next section when determining the locus of minima of certain effective potentials.

\subsection{Useful special functions}

The Pochhammer symbol $(|q| \in] 0,1[)$

$$
(a ; q):=\prod_{k=0}^{\infty}\left(1-a q^{k}\right),
$$

is related to the more familiar Dedekind eta function via

$$
\eta(\tau)=q^{1 / 24}(q ; q)
$$

with $q=e^{2 \pi i \tau}$.

The eta function has an $\mathrm{SL}(2, \mathbb{Z})$ modular property that will be useful for us: $\eta(-1 / \tau)=\sqrt{-i \tau} \eta(\tau)$.

The Pochhammer symbol $(q ; q)$ equals the inverse of the generating function of integer partitions. It also appears in the index of 4d SUSY gauge theories that contain vector multiplets.

The elliptic gamma function is defined as $(\operatorname{Im}(\tau), \operatorname{Im}(\sigma)>0)$

$$
\Gamma(x ; \sigma, \tau):=\prod_{j, k \geq 0} \frac{1-z^{-1} p^{j+1} q^{k+1}}{1-z p^{j} q^{k}},
$$

with $z:=e^{2 \pi i x}, p:=e^{2 \pi i \sigma}=e^{-\beta b}$, and $q:=e^{2 \pi i \tau}=e^{-\beta b^{-1}}$. The above expression gives a meromorphic function of $x \in \mathbb{C}$. For generic choice of $\tau$ and $\sigma$, the elliptic gamma has simple poles at $x=l-m \sigma-n \tau$, with $m, n \in \mathbb{Z} \geq 0, l \in \mathbb{Z}$.

The elliptic gamma function appears in the exact solution of some important $2 \mathrm{~d}$ integrable lattice models. It also features in the index of 4d Lagrangian SUSY QFTs that contain chiral multiplets. 
Following Rains [13], we define the hyperbolic gamma function by

$$
\Gamma_{h}\left(x ; \omega_{1}, \omega_{2}\right):=\exp \left(\mathrm{PV} \int_{\mathbb{R}} \frac{e^{2 \pi i x w}}{\left(e^{2 \pi i \omega_{1} w}-1\right)\left(e^{2 \pi i \omega_{2} w}-1\right)} \frac{\mathrm{d} w}{w}\right) .
$$

The above expression makes sense only for $0<\operatorname{Im}(x)<2 \operatorname{Im}(\omega)$, with $\omega:=\left(\omega_{1}+\omega_{2}\right) / 2$. In that domain, the function defined by (2.4) satisfies

$$
\Gamma_{h}\left(x+\omega_{2} ; \omega_{1}, \omega_{2}\right)=2 \sin \left(\frac{\pi x}{\omega_{1}}\right) \Gamma_{h}\left(x ; \omega_{1}, \omega_{2}\right) .
$$

This relation can then be used for an inductive meromorphic continuation of the hyperbolic gamma function to all $x \in \mathbb{C}$. For generic $\omega_{1}, \omega_{2}$ in the upper half plane, the resulting meromorphic function $\Gamma_{h}\left(x ; \omega_{1}, \omega_{2}\right)$ has simple zeros at $x=\omega_{1} \mathbb{Z}^{\geq 1}+\omega_{2} \mathbb{Z}^{\geq 1}$ and simple poles at $x=\omega_{1} \mathbb{Z} \leq 0+\omega_{2} \mathbb{Z} \leq 0$.

We will encounter the hyperbolic gamma function in the $S_{b}^{3}$ partition function of $3 \mathrm{~d}$ supersymmetric gauge theories which we will obtain from reducing $4 \mathrm{~d}$ gauge theories on the $S^{1}$ of $S_{b}^{3} \times S^{1}$.

\subsection{Some asymptotic analysis}

We say $f(\beta)=O(g(\beta))$ as $\beta \rightarrow 0$, if there exist positive real numbers $C, \beta_{0}$ such that for all $\beta<\beta_{0}$ we have $|f(\beta)|<C|g(\beta)|$. We say $f(x, \beta)=O(g(x, \beta))$ uniformly over $S$ as $\beta \rightarrow 0$, if there exist positive real numbers $C, \beta_{0}$ such that for all $\beta<\beta_{0}$ and all $x \in S$ we have $|f(x, \beta)|<C|g(x, \beta)|$.

We will write $f(\beta)=o(g(\beta))$, if $f(\beta) / g(\beta) \rightarrow 0$ as $\beta \rightarrow 0$.

We use the symbol $\sim$ when writing the all-orders asymptotics of a function. For example, we have

$$
\ln \left(\beta+e^{-1 / \beta}\right) \sim \ln \beta, \quad(\text { as } \beta \rightarrow 0)
$$

because we can write the l.h.s. as the sum of $\ln \beta$ and $\ln \left(1+e^{-1 / \beta} / \beta\right)$, and the latter is beyond all-orders in $\beta$.

More precisely, we say $f(\beta) \sim g(\beta)$ as $\beta \rightarrow 0$, if we have $f(\beta)-g(\beta)=O\left(\beta^{n}\right)$ for any (arbitrarily large) $n$.

We will write $f(\beta) \simeq g(\beta)$ if $\ln f(\beta) \sim \ln g(\beta)$ (with an appropriate choice of branch for the logarithms). By writing $f(x, \beta) \simeq g(x, \beta)$ we mean that $\ln f(x, \beta) \sim \ln g(x, \beta)$ for all $x$ on which $f(x, \beta), g(x, \beta) \neq 0$, and that $f(x, \beta)=g(x, \beta)=0$ for all $x$ on which either $f(x, \beta)=0$ or $g(x, \beta)=0$.

With the above notations at hand, we can asymptotically analyze the Pochhammer symbol as follows. The low-temperature $\left(T \rightarrow 0\right.$, with $\left.q=e^{-1 / T}\right)$ behavior is trivial:

$$
(q ; q) \simeq 1 \quad(\text { as } 1 / \beta \rightarrow 0) .
$$

The high-temperature $\left(\beta \rightarrow 0\right.$, with $\left.q=e^{-\beta}\right)$ asymptotics is nontrivial. It can be obtained using the $\mathrm{SL}(2, \mathbb{Z})$ modular property of the eta function, which yields

$$
\ln \eta\left(\tau=\frac{i \beta}{2 \pi}\right) \sim-\frac{\pi^{2}}{6 \beta}+\frac{1}{2} \ln \left(\frac{2 \pi}{\beta}\right) \quad(\text { as } \beta \rightarrow 0) .
$$



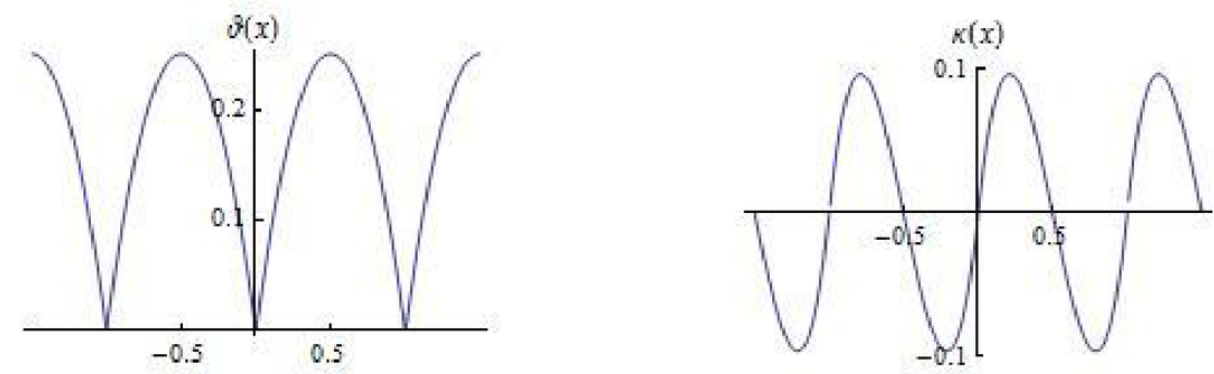

Figure 1. The even function $\vartheta(x)$ (on the left) and the odd function $\kappa(x)$ (on the right). Both are continuous and periodic, and their fundamental domain can be taken to be $[-1 / 2,1 / 2]$.

The above relation, when combined with (2.2), implies

$$
\ln (q ; q) \sim-\frac{\pi^{2}}{6 \beta}+\frac{1}{2} \ln \left(\frac{2 \pi}{\beta}\right)+\frac{\beta}{24} \quad(\text { as } \beta \rightarrow 0) .
$$

Next, we write estimates for the elliptic gamma function. Although its low-temperature asymptotics is not needed for our main purposes below, we encourage the reader without prior familiarity with the elliptic gamma to convince herself that for fixed $r \in] 0,2[$

$$
\frac{1}{\Gamma(x ; \sigma, \tau)} \simeq 1-z, \quad \text { and } \quad \Gamma\left((p q)^{r / 2} z\right) \simeq 1, \quad(\text { as } 1 / \beta \rightarrow 0, \text { for } x \in \mathbb{R})
$$

both valid uniformly over $(x \in) \mathbb{R}$.

The high-temperature asymptotics of the elliptic gamma function is quite nontrivial. From Proposition 2.11 of [13] we obtain the following uniform estimate over $(x \in) \mathbb{R}$ (cf. Proposition 2.12 of [13], with $v_{\text {there }}=\beta_{\text {here }} / 2 \pi$; see also appendix A):

$$
\ln \Gamma\left(x+r\left(\frac{\sigma+\tau}{2}\right) ; \sigma, \tau\right)=2 \pi i\left(-\frac{\kappa(x)}{12 \tau \sigma}+(r-1) \frac{\tau+\sigma}{4 \tau \sigma} \vartheta(x)-(r-1) \frac{\tau+\sigma}{24 \tau \sigma}\right)+O\left(\beta^{0}\right)
$$

(for fixed $r \in] 0,2[$ ).

Following Rains [13], we have defined the continuous, positive, even, periodic function ${ }^{2}$

$$
\begin{aligned}
\vartheta(x) & :=\{x\}(1-\{x\}) \\
( & \left.=|x|-x^{2} \quad \text { for } x \in[-1,1]\right),
\end{aligned}
$$

with $\{x\}:=x-\lfloor x\rfloor$. We have also introduced the continuous, odd, periodic function

$$
\begin{aligned}
\kappa(x) & :=\{x\}(1-\{x\})(1-2\{x\}) \\
& \left(=2 x^{3}-3 x|x|+x \quad \text { for } x \in[-1,1]\right) .
\end{aligned}
$$

These functions are displayed in figure 1.

The real number $r$ in (2.11) will be interpreted in the next section as the R-charge of a chiral multiplet.

\footnotetext{
${ }^{2}$ This function is closely related to the functions $1-[x]_{+}^{2}$ (in appendix D of [42]) and $g(x)$ (in appendix A of [43]) appearing in the context of perturbative corrections to low-energy effective actions on $R^{3} \times S^{1}$.
} 
To analyze the SUSY partition function of theories that contain vector multiplets, we will need an estimate similar to (2.11) that would apply when $r=0$. The following asymptotic relation, valid uniformly over compact subsets of $\mathbb{R}$ avoiding an $O(\beta)$ neighborhood of $\mathbb{Z}$, gives the desired estimate (cf. Proposition 2.12 of [13]):

$$
\ln \left(\frac{1}{\Gamma(x ; \sigma, \tau) \Gamma(-x ; \sigma, \tau)}\right)=2 \pi i\left(\frac{\tau+\sigma}{2 \tau \sigma} \vartheta(x)-\frac{\tau+\sigma}{12 \tau \sigma}\right)+O\left(\beta^{0}\right) .
$$

Note that the above relation would follow from a (sloppy) use of (2.11) with $r=0$. But unlike (2.11), the above estimate is not valid uniformly over $\mathbb{R}$. For real $x$ in an $O(\beta)$ neighborhood of $\mathbb{Z}$, the following slightly weaker version of (2.14) applies (cf. Corollary 3.1 of [13]):

$$
\frac{1}{\Gamma(x ; \sigma, \tau) \Gamma(-x ; \sigma, \tau)}=O\left(\exp \left[2 \pi i\left(\frac{\tau+\sigma}{2 \tau \sigma} \vartheta(x)-\frac{\tau+\sigma}{12 \tau \sigma}\right)\right]\right) \quad(\text { as } \beta \rightarrow 0) .
$$

(A stronger estimate in this region can be obtained by relating the product on the l.h.s. to a product of theta functions, and then using the modular property of the theta function.)

The reason (2.15) is weaker than (2.14) is roughly that the argument of $O$ on the r.h.s. of (2.15) is an overestimate of the l.h.s., in particular (as the reader can easily check) for $x \in \mathbb{Z}$.

Since the estimates (2.11), (2.14), and (2.15) encode only the leading asymptotics of the elliptic gamma function, we refer to them as the "leading estimates". These estimates alone will suffice for obtaining the effective potential on the moduli space of the holonomies in the next section.

A much more precise estimate for the elliptic gamma function is (cf. Proposition 2.10 in $[13])$

$$
\begin{array}{r}
\ln \Gamma\left((p q)^{r / 2} z ; p, q\right) \sim 2 \pi i R_{0}\left(x+r\left(\frac{\tau+\sigma}{2}\right) ; \sigma, \tau\right)+\ln \Gamma_{h}\left(\frac{2 \pi x}{\beta}+\omega r ; \omega_{1}, \omega_{2}\right) \\
(\text { as } \beta \rightarrow 0, \text { for } x \in]-1,1[)
\end{array}
$$

valid uniformly over (fixed, $\beta$-independent) compact subsets of the domain ] - 1, 1[, assuming $r \in] 0,2[$ is fixed, and with

$$
R_{0}(x ; \sigma, \tau):=-\frac{x^{3}}{6 \tau \sigma}+\frac{\tau+\sigma}{4 \tau \sigma} x^{2}-\frac{\tau^{2}+\sigma^{2}+3 \tau \sigma+1}{12 \tau \sigma} x+\frac{1}{24} \frac{\tau+\sigma}{\tau \sigma}+\frac{1}{24}(\tau+\sigma) .
$$

The restriction we imposed on the range of $r$ ensures that the (log of the) hyperbolic gamma function on the r.h.s. of (2.16) is well-defined at $x=0$.

The domain of validity of (2.16) can be easily extended from $x \in]-1,1[$ to $x \in \mathbb{R}$, by replacing every $x$ on the r.h.s. with $\{x\}$. This is because the l.h.s. of (2.16) is a function of $z$, and $z=e^{2 \pi i x}$ is invariant under $x \rightarrow x+1$.

At $x=0$, the physical content of the relation (2.16) is the well-known fact that the superconformal index of a free $4 \mathrm{~d}$ chiral multiplet (the elliptic gamma on the l.h.s.) reduces at high temperatures to the squashed-three-sphere partition function of the $3 \mathrm{~d}$ 
chiral multiplet (the hyperbolic gamma on the r.h.s.) obtained by reducing the $4 \mathrm{~d}$ multiplet on $S^{1}$.

The vector-multiplet analog of (2.16) reads

$$
\frac{1}{\Gamma\left(z^{ \pm 1}\right)} \simeq \frac{e^{-2 \pi i\left[R_{0}(x ; \sigma, \tau)+R_{0}(-x ; \sigma, \tau)\right]}}{\Gamma_{h}\left( \pm \frac{2 \pi x}{\beta}\right)}, \quad(\text { as } \beta \rightarrow 0, \text { for } x \in]-1,1[)
$$

which is valid uniformly over (fixed, $\beta$-independent) compact subsets of the domain ] $-1,1[$. Note that a (sloppy) use of (2.16) for $r=0$ would have yielded (2.18) correctly.

We refer to (2.16) and (2.18) as the "central estimates" for the elliptic gamma function.

Finally, we will need the asymptotics of the hyperbolic gamma function. Corollary 2.3 of [13] implies that for $x \in \mathbb{R}$

$$
\ln \Gamma_{h}\left(x+r \omega ; \omega_{1}, \omega_{2}\right)=-\frac{i \pi}{2} x|x|-i \pi(r-1) \omega|x|+O(1), \quad(\text { as }|x| \rightarrow \infty)
$$

for any fixed real $r$, and fixed $b>0$. The above relation allows us to determine, among other things, whether or not the three-sphere partition functions we obtain from the hightemperature limit of SUSY partition functions are finite.

\subsection{Generalized triangle inequalities}

After using the estimates (2.11) and (2.19), we will need some relations that the functions $\vartheta(x)$ and $|x|$ satisfy.

The most important relation, which yields several others as corollaries, is Rains's generalized triangle inequality. The Lemma 3.2 of [13] says that for any sequence of real numbers $c_{1}, \ldots, c_{n}, d_{1}, \ldots, d_{n}$, the following inequality holds:

$$
\sum_{1 \leq i, j \leq n} \vartheta\left(c_{i}-d_{j}\right)-\sum_{1 \leq i<j \leq n} \vartheta\left(c_{i}-c_{j}\right)-\sum_{1 \leq i<j \leq n} \vartheta\left(d_{i}-d_{j}\right) \geq \vartheta\left(\sum_{1 \leq i \leq n}\left(c_{i}-d_{i}\right)\right),
$$

with equality iff the sequence can be permuted so that either

$$
\left\{c_{1}\right\} \leq\left\{d_{1}\right\} \leq\left\{c_{2}\right\} \leq \cdots \leq\left\{d_{n-1}\right\} \leq\left\{c_{n}\right\} \leq\left\{d_{n}\right\},
$$

or

$$
\left\{d_{1}\right\} \leq\left\{c_{1}\right\} \leq\left\{d_{2}\right\} \leq \cdots \leq\left\{c_{n-1}\right\} \leq\left\{d_{n}\right\} \leq\left\{c_{n}\right\} .
$$

The proof can be found in [13].

Re-scaling with $c_{i}, d_{i} \mapsto v c_{i}, v d_{i}$, taking $v \rightarrow 0^{+}$, and using the relation $\vartheta(v x)=$ $v|x|-v^{2} x^{2}$ (which holds for small enough $v$ ), Rains obtains the following corollary of (2.20):

$$
\sum_{1 \leq i, j \leq n}\left|c_{i}-d_{j}\right|-\sum_{1 \leq i<j \leq n}\left|c_{i}-c_{j}\right|-\sum_{1 \leq i<j \leq n}\left|d_{i}-d_{j}\right| \geq\left|\sum_{1 \leq i \leq n}\left(c_{i}-d_{i}\right)\right|,
$$

with equality iff the sequence can be permuted so that either

$$
c_{1} \leq d_{1} \leq c_{2} \leq \cdots \leq d_{n-1} \leq c_{n} \leq d_{n}
$$

or

$$
d_{1} \leq c_{1} \leq d_{2} \leq \cdots \leq c_{n-1} \leq d_{n} \leq c_{n}
$$




\section{Asymptotics of the SUSY partition function}

The SUSY partition function of a supersymmetric gauge theory with a semi-simple gauge group $G$ (which we think of as a compact matrix Lie group) is given by $[9,10,28]$ (see also $[27,44])$

$$
Z^{\mathrm{SUSY}}(b, \beta)=e^{-\beta E_{\text {susy }}(b)} \mathcal{I}(b, \beta),
$$

with the Romelsberger index of the SUSY gauge theory obtained from

$$
\mathcal{I}(b, \beta)=\frac{(p ; p)^{r_{G}}(q ; q)^{r_{G}}}{|W|} \int \mathrm{d}^{r_{G}} x \frac{\prod_{\chi} \prod_{\rho \chi \in \Delta_{\chi}} \Gamma\left((p q)^{r_{\chi} / 2} z^{\rho^{\chi}}\right)}{\prod_{\alpha_{+}} \Gamma\left(z^{ \pm \alpha_{+}}\right)},
$$

and the SUSY Casimir energy [28] given by

$$
E_{\text {susy }}(b)=\frac{i}{6} \operatorname{Tr}[R \omega]^{3}+i\left(\frac{b^{2}+b^{-2}}{24}\right) \operatorname{Tr}[R \omega] .
$$

In (3.2), $p=e^{-\beta b}, q=e^{-\beta b^{-1}}$, with $\left.\beta, b \in\right] 0, \infty[$. The rank of the gauge group is denoted by $r_{G}$. The $r_{\chi}$, which we assume to be in the interval $] 0,2[$, are the R-charges of chiral multiplets $\chi$ in the theory. The chiral multiplets sit in representations $\mathcal{R}_{\chi}$ of the gauge group, whose set of weights we have denoted by $\Delta_{\chi}$. The set $\Delta_{\chi}$ consists of as many weights $\rho^{\chi}$ as the dimension of the representation $\mathcal{R}_{\chi}$. Our symbolic notation $z^{\rho^{\chi}}$ should be understood as $z_{1}^{\rho_{1}^{\chi}} \times \cdots \times z_{r_{G}}^{\rho_{r_{G}}^{\chi}}$, where $\rho^{\chi} \equiv\left(\rho_{1}^{\chi}, \ldots, \rho_{r_{G}}^{\chi}\right)$. The $\alpha_{+}$are the positive roots of $G$, and $|W|$ is the order of the Weyl group of $G$. The integral is over $x_{i} \in[-1 / 2,1 / 2]$ (or alternatively, over the maximal torus of $G$ in the space of $\left.z_{i}=e^{2 \pi i x_{i}}\right)$. Note that a given positive root is determined by $r_{G}$ numbers $\left(\alpha_{1}, \ldots, \alpha_{r_{G}}\right)$; by $z^{\alpha_{+}}$we mean $z_{1}^{\alpha_{1}} \times \cdots \times z_{r_{G}}^{\alpha_{r_{G}}}$.

The numerator of the integrand of (3.2) comes from the chiral multiplets. The denominator of the integrand together with the prefactor of the integral can be thought of as the contribution of the vector multiplet(s).

Eq. (3.1) describes the way to arrive at the index via the "Lagrangian" path-integral that defines $Z^{\mathrm{SUSY}}(b, \beta)$. There is an alternative "Hamiltonian" route to the index via

$$
\mathcal{I}(b, \beta)=\operatorname{Tr}\left[(-1)^{F} e^{-\hat{\beta}\left(\Delta-2 j_{2}-\frac{3}{2} r\right)} p^{j_{1}+j_{2}+\frac{1}{2} r} q^{-j_{1}+j_{2}+\frac{1}{2} r}\right] .
$$

The trace in the above relation is over the Hilbert space of the theory on $S^{3} \times \mathbb{R}$, with $S^{3}$ the unit round three-sphere, and $\mathbb{R}$ the time direction. The quantum numbers $\left(j_{1}, j_{2}\right)$ label the charges of a state under the Cartan of the $\mathrm{SU}(2)_{1} \times \mathrm{SU}(2)_{2}$ isometry group of $S^{3}$, while the R-charge is denoted $r$, and $\Delta$ - which coincides with the conformal dimension in a superconformal theory - is as in [5]. The index is independent of $\hat{\beta}$, because it only receives contributions from states with $\Delta-2 j_{2}-\frac{3}{2} r=0$. In a superconformal theory, these states correspond to operators that sit in short representations of the superconformal algebra. The index - or alternatively the SUSY partition function - of an SCFT thus encodes exact (non-perturbative) information about the operator spectrum of the underlying theory.

Since the expression in eq. (3.2) might seem a bit complicated, let us specialize it to a very simple case: the SU(2) SQCD with three flavors. The gauge group SU(2) has rank 
$r_{G}=1$. The Weyl group of $\mathrm{SU}(N)$ is the permutation group of $N$ elements, so it has order $N$ !, which for $\mathrm{SU}(2)$ becomes 2 . We have three chiral quark multiplets with $\rho_{1}^{\chi_{1}}, \rho_{1}^{\chi_{2}}, \rho_{1}^{\chi_{3}}=$ \pm 1 , and three chiral anti-quark multiplets with $\rho_{1}^{\chi_{4}}, \rho_{1}^{\chi_{5}}, \rho_{1}^{\chi_{6}}=\mp 1$ (each of the chiral multiplets has two weights $( \pm 1)$, because they sit in two-dimensional representations of the gauge group). All the chiral multiplets have R-charge $r_{\chi}=1 / 3$. Finally, the group $\mathrm{SU}(2)$ has two roots, corresponding to the raising and lowering operators of the $3 \mathrm{~d}$ angular momentum, and the positive root (the raising operator) has $\alpha_{+}=2$. All in all, we get for this simple example

$$
Z_{N_{c}=2, N_{f}=3}^{\mathrm{SUSY}}(b, \beta)=e^{-\beta E_{\text {susy }}(b)} \frac{(p ; p)(q ; q)}{2} \int_{-1 / 2}^{1 / 2} \mathrm{~d} x \frac{\Gamma^{6}\left((p q)^{1 / 6} z^{ \pm 1}\right)}{\Gamma\left(z^{ \pm 2}\right)},
$$

where $E_{\text {susy }}(b)=i \frac{\omega^{3}}{6} \operatorname{Tr} R^{3}+i\left(\frac{b^{2}+b^{-2}}{24}\right) \omega \operatorname{Tr} R$, with $\operatorname{Tr} R^{3}=-5 / 9$ and $\operatorname{Tr} R=-5$. The interested reader is invited to show, using the low-temperature estimates (2.7) and (2.10), that as $1 / \beta \rightarrow 0$ the partition function is dominated by the vacuum energy: $Z_{N_{c}=2, N_{f}=3}^{\mathrm{SUSY}}(b, \beta) \simeq$ $e^{-\beta E_{\text {susy }}(b)}$.

We will spell out the SUSY partition function of several other theories below. The reader can also consult $[11,12,23,25]$ wherein explicit expressions are given for the indices of many more physically interesting supersymmetric QFTs.

As a warm-up for our high-temperature analysis, let's study the low-temperature asymptotics of the SUSY partition function (3.1). The estimates (2.7) and (2.10) (the latter being valid assuming $\left.r_{\chi} \in\right] 0,2[)$ simplify the index (3.2) at low temperatures as

$$
\mathcal{I}(b, \beta) \simeq \frac{1}{|W|} \int \mathrm{d}^{r_{G}} x \prod_{\alpha_{+}}\left(\left(1-z^{\alpha_{+}}\right)\left(1-z^{-\alpha_{+}}\right)\right)=1, \quad(\text { as } 1 / \beta \rightarrow 0)
$$

with the equality on the r.h.s. resulting from the Weyl integral formula. The relation (3.1) then yields the universal low-temperature asymptotics

$$
Z^{\mathrm{SUSY}}(b, \beta) \simeq e^{-\beta E_{\text {susy }}(b)} \quad(\text { as } 1 / \beta \rightarrow 0, \text { with } b \text { fixed }) .
$$

Recall that the symbol $\simeq$ indicates equality to all orders - in $1 / \beta$ - after taking the logarithm of both sides.

A relation similar to (3.7) holds for an equivariant generalization of $Z^{\mathrm{SUSY}}(b, \beta)$, which we denote by $Z^{\mathrm{SUSY}}\left(b, \beta ; m_{a}\right)$. The latter is computed in presence of real background gauge fields $m_{a}$ along $S_{\beta}^{1}$, each coupling a conserved $\mathrm{U}(1)_{a}$ current in the theory; setting all $m_{a}$ to zero, we recover $Z^{\mathrm{SUSY}}(b, \beta)$. In that relation, $E_{\text {susy }}(b)$ is replaced with $E_{\text {susy }}\left(b ; m_{a}\right)$ which can be obtained from (3.3) by shifting every $R \omega$ in it to $R \omega+Q_{a} m_{a}$, with $Q_{a}$ the $\mathrm{U}(1)_{a}$ charge of the chiral fermions in the theory. As emphasized in [26], $E_{\text {susy }}\left(b ; m_{a}\right)$ contains complete information about the linear and cubic 't Hooft anomalies of the SUSY QFT. Therefore (if all $r_{\chi}$ are in $] 0,2[$ ) the ' $\mathrm{t}$ Hooft anomaly matching conditions correspond to matching the low-temperature asymptotics of the equivariant SUSY partition functions of dual SUSY QFTs. [As we will discuss in subsections 3.1, 3.2, and 3.3 below, various 't Hooft anomalies appear also in the high-temperature asymptotics of $Z^{\mathrm{SUSY}}\left(b, \beta ; m_{a}\right)$, but their appearance is not as universal as in $E_{\text {susy }}\left(b ; m_{a}\right)$.] 
We now get to the main subject of the present section. The high-temperature asymptotics of the partition function in (3.1) is found as follows. The Casimir energy factor is of course negligible at the leading order. The Pochhammer symbols in the prefactor of (3.2) can be immediately replaced with their asymptotic expressions obtainable from (2.9). Focusing on the divergent asymptotics we have

$$
(p ; p)^{r_{G}}(q ; q)^{r_{G}} \approx e^{-\pi^{2}\left(b+b^{-1}\right) r_{G} / 6 \beta} \times\left(\frac{2 \pi}{\beta}\right)^{r_{G}},
$$

which is accurate to within a multiplicative $O\left(\beta^{0}\right)$ factor.

The leading asymptotics of the integrand of (3.2) can be obtained from the leading estimates (2.11) and (2.14). Combining these two estimates with (3.8), we find that $Z^{\mathrm{SUSY}}$ and $\mathcal{I}$ simplify at high temperatures to

$$
Z^{\mathrm{SUSY}}(b, \beta) \approx \mathcal{I}(b, \beta) \approx\left(\frac{2 \pi}{\beta}\right)^{r_{G}} \int_{\mathfrak{h}_{c l}} \mathrm{~d}^{r_{G}} x e^{-\left[\mathcal{E}_{0}^{D K}(b, \beta)+V^{\mathrm{eff}}(\mathbf{x} ; b, \beta)\right]+i \Theta(\mathbf{x} ; \beta)},
$$

with $\mathfrak{h}_{c l}$ the unit hypercube $x_{i} \in[-1 / 2,1 / 2]$, and with

$$
\begin{aligned}
\mathcal{E}_{0}^{D K}(b, \beta) & =-i \frac{\pi^{2}}{3 \beta} \operatorname{Tr}[R \omega], \\
V^{\mathrm{eff}}(\mathbf{x} ; b, \beta) & =\frac{4 \pi^{2}}{\beta}\left(\frac{b+b^{-1}}{2}\right) L_{h}(\mathbf{x}), \\
\Theta(\mathbf{x} ; \beta) & =\frac{8 \pi^{3}}{\beta^{2}} Q_{h}(\mathbf{x}) .
\end{aligned}
$$

For convenience we have introduced $\mathbf{x}:=\left(x_{1}, \ldots, x_{r_{G}}\right)$. The real functions $Q_{h}(\mathbf{x})$ and $L_{h}(\mathbf{x})$ are defined by

$$
\begin{aligned}
Q_{h}(\mathbf{x}) & :=\frac{1}{12} \sum_{\chi} \sum_{\rho^{\chi} \in \Delta_{\chi}} \kappa\left(\left\langle\rho^{\chi} \cdot \mathbf{x}\right\rangle\right), \\
L_{h}(\mathbf{x}) & :=\frac{1}{2} \sum_{\chi}\left(1-r_{\chi}\right) \sum_{\rho^{\chi} \in \Delta_{\chi}} \vartheta\left(\left\langle\rho^{\chi} \cdot \mathbf{x}\right\rangle\right)-\sum_{\alpha_{+}} \vartheta\left(\left\langle\alpha_{+} \cdot \mathbf{x}\right\rangle\right) .
\end{aligned}
$$

Note that in (3.9) we are claiming that the matrix-integral is approximated well with the integral of its approximate integrand. This is not entirely obvious. First of all, while the estimate (2.11) for the chiral-multiplet gamma functions is valid uniformly over the domain of integration, the estimate (2.14) for the vector-multiplet gamma functions is uniform only over compact subsets of $\mathfrak{h}_{c l}$ that avoid an $O(\beta)$ neighborhood of the Stiefel diagram

$$
\mathcal{S}_{g}:=\bigcup_{\alpha_{+}}\left\{\mathbf{x} \in \mathfrak{h}_{c l} \mid\left\langle\alpha_{+} \cdot \mathbf{x}\right\rangle \in \mathbb{Z}\right\}
$$

Let's denote this neighborhood by $\mathcal{S}_{g}^{(\beta)}$. Intuitively speaking, we expect the estimate (2.15), which applies also on $\mathcal{S}_{g}^{(\beta)}$, to guarantee that our unreliable use of (2.14) over this small region modifies the asymptotics ${ }^{3}$ at most by a multiplicative $O(1)$ factor. This is also the

\footnotetext{
${ }^{3}$ A stronger version of (2.15) implies that the expression (3.14) for $L_{h}$ should be corrected on $\mathcal{S}_{g}^{(\beta)}$. The correction is negligible $\left(o\left(\beta^{0}\right)\right)$ though, except in an $O\left(e^{-1 / \beta}\right)$ neighborhood of $\mathcal{S}_{g}$. In particular, the corrected $L_{h}$ diverges on $\mathcal{S}_{g}$, as the integrand of (3.2) vanishes there.
} 
error of the estimates used in deriving (3.9) from (3.2). Therefore the logarithms of the two sides of the symbols $\approx$ in (3.9) are equal up to an $O\left(\beta^{0}\right)$ error. When $\Theta=Q_{h}=0$, the claim in the previous sentence can be justified more carefully, as we outline below (3.30).

Aside from the issue of non-uniform estimates discussed in the previous paragraph, a second subtlety may arise in going from an estimate of the integrand to an estimate for the integral: cancelations may occur in the actual integral, that do not occur when integrating the estimated integrand; if this happens, the r.h.s. of (3.9) would overestimate the 1.h.s.. Such an overestimation would be symptomized by divergent corrections that would arise when trying to improve (3.9) to higher accuracy. When $\Theta=Q_{h}=0$, the absence of such subtleties is equivalent to the finiteness of the $O\left(\beta^{0}\right)$ term on the r.h.s. of (3.30) (see the discussion below (3.82) for instance); we check this finiteness in some of our explicit examples below, but it can be more generally demonstrated for non-chiral theories (cf. [45]).

In short, (3.9) is demonstrably valid - up to an $O\left(\beta^{0}\right)$ error upon taking the logarithm of the two sides - in non-chiral theories (which have $Q_{h}=0$ ); we leave its validity - up to the said error - for chiral theories (which may have $Q_{h} \neq 0$ ) as a conjecture.

Studying the small- $\beta$ behavior of the multiple-integral on the r.h.s. of (3.9) is now an exercise (albeit a quite nontrivial one) in asymptotic analysis. Before explaining the result, we comment on some important properties of the functions $Q_{h}$ and $L_{h}$ introduced above.

The real function $Q_{h}$ appearing in the phase $\Theta(\mathbf{x} ; \beta)$ is piecewise quadratic, because the cubic terms in it cancel thanks to the gauge-gauge-gauge anomaly cancelation condition:

$$
\frac{\partial^{3} Q_{h}(\mathbf{x})}{\partial x_{i} \partial x_{j} \partial x_{k}}=\sum_{\chi} \sum_{\rho \chi \in \Delta_{\chi}} \rho_{i}^{\chi} \rho_{j}^{\chi} \rho_{k}^{\chi}=0 .
$$

Moreover, as a consequence of the vanishing of the gauge-gravitational-gravitational anomaly, $Q_{h}$ is stationary at the origin:

$$
\left.\frac{\partial Q_{h}(\mathbf{x})}{\partial x_{i}}\right|_{\mathbf{x}=0}=\frac{1}{12} \sum_{\chi} \sum_{\rho^{\chi} \in \Delta_{\chi}} \rho_{i}^{\chi}=0 .
$$

We leave it to the interested reader to verify that $Q_{h}(\mathbf{x})$ has a continuous first derivative. Also, $Q_{h}(\mathbf{x})$ is odd under $\mathbf{x} \rightarrow-\mathbf{x}$, and vanishes at $\mathbf{x}=0$; these properties follow from the fact that the function $\kappa(x)$ defined in (2.13) is a continuous odd function of its argument. As a result of its oddity, $Q_{h}(\mathbf{x})$ identically vanishes if the nonzero $\rho^{\chi}$ come in pairs with opposite signs; we refer to theories with such matter content as non-chiral; most of the specific examples that we study in the present paper are of this kind.

When all $x_{i}$ are small enough, so that the absolute value of all the arguments of the $\kappa$ functions in $Q_{h}$ are less than 1 , we can use $\kappa(x)=2 x^{3}-3 x|x|+x$ to simplify $Q_{h}$. The resulting expression - which equals $Q_{h}$ for $x_{i}$ small enough — can then be considered as defining a function $\tilde{Q}_{S^{3}}(x)$ for any $x_{i} \in \mathbb{R}$. Explicitly, we have

$$
\tilde{Q}_{S^{3}}(\mathbf{x})=-\frac{1}{4} \sum_{\chi} \sum_{\rho \chi \in \Delta_{\chi}}\left\langle\rho^{\chi} \cdot \mathbf{x}\right\rangle\left|\left\langle\rho^{\chi} \cdot \mathbf{x}\right\rangle\right|,
$$


with no linear or cubic terms thanks to the cancelation of the gauge-gravitationalgravitational and gauge-gauge-gauge anomalies. The homogeneity of $\tilde{Q}_{S^{3}}$ will be important for us below. The reason for the subscript $S^{3}$ will become clear shortly.

The star of our show, the real function $L_{h}$, determines the effective potential ${ }^{4}$ $V^{\mathrm{eff}}(\mathbf{x} ; b, \beta)$. It is piecewise linear; the quadratic terms in it cancel because of the ABJ

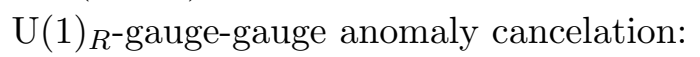

$$
\frac{\partial^{2} L_{h}(\mathbf{x})}{\partial x_{i} \partial x_{j}}=\sum_{\chi}\left(r_{\chi}-1\right) \sum_{\rho \chi \in \Delta_{\chi}} \rho_{i}^{\chi} \rho_{j}^{\chi}+\sum_{\alpha} \alpha_{i} \alpha_{j}=0 .
$$

Also, $L_{h}$ is continuous, is even under $\mathbf{x} \rightarrow-\mathbf{x}$, and vanishes at $\mathbf{x}=0$; these properties follow from the properties of the function $\vartheta(x)$ defined in (2.12). We refer to $L_{h}(\mathbf{x})$ as the Rains function of the gauge theory. This function has been analyzed in [13] in the context of the elliptic hypergeometric integrals associated to $\mathrm{SU}(N)$ and $\operatorname{Sp}(N) \operatorname{SQCD}$ theories.

When all $x_{i}$ are small enough, such that the absolute value of the argument of every $\vartheta$ function in $L_{h}$ is smaller than 1 , we can use $\vartheta(x)=|x|-x^{2}$ to simplify the Rains function. The resulting expression - which equals $L_{h}$ for small $x_{i}$ - can then be considered as defining a function $\tilde{L}_{S^{3}}(\mathbf{x})$ for any $x_{i} \in \mathbb{R}$. Explicitly, we have ${ }^{5}$

$$
\tilde{L}_{S^{3}}(\mathbf{x})=\frac{1}{2} \sum_{\chi}\left(1-r_{\chi}\right) \sum_{\rho^{\chi} \in \Delta_{\chi}}\left|\left\langle\rho^{\chi} \cdot \mathbf{x}\right\rangle\right|-\sum_{\alpha_{+}}\left|\left\langle\alpha_{+} \cdot \mathbf{x}\right\rangle\right| .
$$

Note that there is no quadratic term in $\tilde{L}_{S^{3}}$, thanks to the cancelation of the U(1) $R_{R^{-} \text {gauge- }}$ gauge anomaly. The homogeneity of $\tilde{L}_{S^{3}}$ will be important for us below. We have added a subscript $S^{3}$ in $\tilde{L}_{S^{3}}$, because this function plays an important role in determining whether or not the $S^{3}$ partition function of the gauge theory reduced on $S_{\beta}^{1}$ is finite.

The high-temperature analysis of the integral (3.9) proceeds as follows. First take the factor $e^{-\mathcal{E}_{0}^{D K}(b, \beta)}$ outside the integral. Then, since the real part of the exponent of the integrand is proportional to $L_{h}(\mathbf{x}) / \beta$, the $\beta \rightarrow 0$ limit localizes the integral to the locus of the minima of $L_{h}(\mathbf{x})$, and the integrand is asymptotically exponentially suppressed away from the locus. This argument suggests, though does not prove, the high-temperature localization of $Z^{\mathrm{SUSY}}(b, \beta)$. (A rigorous analysis must first resolve the tension between minimizing $V^{\text {eff }}$ and making $\Theta$ stationary.)

\footnotetext{
${ }^{4}$ Somewhat surprisingly, $L_{h}$ also appears in the $n \rightarrow 1$ limit of the zero-point energy associated to nonzero spatial holonomies on $S^{1} \times S^{3} / \mathbb{Z}_{n}$; cf. eq. (29) of the arXiv preprint of [46] (with $\nu, a$ in there set to zero). It might be possible to clarify this coincidence by analytically continuing the results of [46] (see also $[47,48]$ ) to non-integer $n$, and then using modular properties of the generalized elliptic gamma functions employed in that work.

${ }^{5}$ Interestingly, on a discrete subset of its domain (corresponding to the cocharacter lattice of the gauge group $G$ ), the function $\tilde{L}_{S^{3}}$ coincides (up to normalization) with the $S^{2} \times S^{1}$ Casimir energy $\epsilon_{0}$ [49] associated to monopole sectors of the $3 \mathrm{~d} \mathcal{N}=2$ theory obtained from dimensional reduction of the $4 \mathrm{~d}$ gauge theory. Similarly, $b_{0}(a)$ in [49] is related to $\tilde{Q}_{S^{3}}$ above. Also, an analog of the $q_{0 i}$ of that work would appear in our analysis if we turn on equivariant parameters. The observation in the previous footnote might provide a clue for understanding this set of coincidences. In the context of $3 \mathrm{~d} \mathcal{N}=4$ theories, a different connection between $\tilde{L}_{S^{3}}$ and 3 d monopoles was discussed in [39].
} 
To make more precise statements, we now focus on the cases where $\Theta=0$; more specifically, we will keep non-chiral theories in mind. The cases with $\Theta \neq 0$ (hence with chiral matter content) require more care, and will not be treated in generality here; we will study a couple of such examples below.

Setting $\Theta=0$, and writing $V^{\text {eff }}$ in terms of the Rains function $L_{h},(3.9)$ simplifies to $Z^{\mathrm{SUSY}}(b, \beta) \approx\left(\frac{2 \pi}{\beta}\right)^{r_{G}} e^{-\mathcal{E}_{0}^{D K}(b, \beta)} \int_{\mathfrak{h}_{c l}} \mathrm{~d}^{r_{G}} x e^{-\frac{4 \pi^{2}}{\beta}\left(\frac{b+b^{-1}}{2}\right) L_{h}(\mathbf{x})} \quad\left(\right.$ as $\beta \rightarrow 0$, when $\left.Q_{h}=0\right)$.

The asymptotic small- $\beta$ analysis of the above integral is straightforward, but somewhat detailed. Therefore we first give a brief outline of how the analysis proceeds and what the final result looks like. The integral localizes, as $\beta \rightarrow 0$, around the locus of the minima of $L_{h}$. This locus is a subset of $\mathfrak{h}_{c l}$ that we denote by $\mathfrak{h}_{q u}$, and write its dimension as dimh $\mathfrak{h}_{q u}$. The integration goes over $\operatorname{dim} \mathfrak{h}_{q u}$ directions along $\mathfrak{h}_{q u}$, and $r_{G}-\operatorname{dimh} \mathfrak{h}_{q u}$ directions perpendicular to it. Along the directions perpendicular to $\mathfrak{h}_{q u}$, the integrand decays exponentially; to get an order one (instead of $O\left(\beta^{r_{G}-\operatorname{dimh}_{q u}}\right)$ ) result from integrating along them, it turns out that one has to absorb $r_{G}-\operatorname{dimh}_{q u}$ factors of $2 \pi / \beta$ into the integral. This leaves $\operatorname{dim} \mathfrak{h}_{q u}$ factors of $2 \pi / \beta$, besides the exponential factors that we have already seen in eq. (1.5) of the introduction. The end result is displayed in eq. (3.30). The reader not interested in a more careful derivation of that result is invited to continue reading from eq. (3.30), and skip the detailed analysis below.

To analyze the integral in (3.21) more carefully, first note that the integrand is not smooth over $\mathfrak{h}_{c l}$. We therefore break $\mathfrak{h}_{c l}$ into sets on which $L_{h}$ is linear. These sets can be obtained as follows. Define

$$
\mathcal{S}_{\chi}:=\bigcup_{\rho^{\chi} \in \Delta_{\chi}^{\neq 0}}\left\{\mathbf{x} \in \mathfrak{h}_{c l} \mid\left\langle\rho^{\chi} \cdot \mathbf{x}\right\rangle \in \mathbb{Z}\right\}, \quad \mathcal{S}:=\bigcup_{\chi} \mathcal{S}_{\chi} \cup \mathcal{S}_{g}
$$

with $\Delta_{\chi}^{\neq 0}\left(\subset \Delta_{\chi}\right)$ the set of nonzero weights of $\mathcal{R}_{\chi}$. Note that everywhere in $\mathfrak{h}_{c l}$, except on $\mathcal{S}$, the function $L_{h}$ is guaranteed to be linear - and therefore smooth.

The set $\mathcal{S}$ consists of a union of codimension one affine hyperplanes inside the space of the $x_{i}$. These hyperplanes chop $\mathfrak{h}_{c l}$ into (finitely many, convex) polytopes $\mathcal{P}_{n}$. The integral in (3.21) then decomposes to

$$
Z^{\mathrm{SUSY}}(b, \beta) \approx \sum_{n}\left(\frac{2 \pi}{\beta}\right)^{r_{G}} e^{-\mathcal{E}_{0}^{D K}(b, \beta)} \int_{\mathcal{P}_{n}} \mathrm{~d}^{r_{G}} x e^{-\frac{4 \pi^{2}}{\beta}\left(\frac{b+b^{-1}}{2}\right) L_{h}(\mathbf{x})} .
$$

Since $L_{h}$ is linear on each $\mathcal{P}_{n}$, its minimum over $\mathcal{P}_{n}$ is guaranteed to be realized on $\partial \mathcal{P}_{n}$. Let us assume that this minimum occurs on the $k$ th $j$-face of $\mathcal{P}_{n}$, which we denote by $j_{n}-\mathcal{F}_{n}^{k}$. We denote the value of $L_{h}$ on this $j$-face by $L_{h \text { min }}^{n}$. Equipped with these notations, we can write $(3.23)$ as

$$
Z^{\mathrm{SUSY}}(b, \beta) \approx \sum_{n}\left(\frac{2 \pi}{\beta}\right)^{r_{G}} e^{-\mathcal{E}_{0}^{D K}(b, \beta)-\frac{4 \pi^{2}}{\beta}\left(\frac{b+b^{-1}}{2}\right) L_{h \min }^{n}} \int_{\mathcal{P}_{n}} \mathrm{~d}^{r_{G}} x e^{-\frac{4 \pi^{2}}{\beta}\left(\frac{b+b^{-1}}{2}\right) \Delta L_{h}^{n}(\mathbf{x})},
$$


where $\Delta L_{h}^{n}(\mathbf{x}):=L_{h}(\mathbf{x})-L_{h \text { min }}^{n}$ is a linear function on $\mathcal{P}_{n}$. Note that $\Delta L_{h}^{n}(\mathbf{x})$ vanishes on $j_{n}-\mathcal{F}_{n}^{k}$, and it increases as we go away from $j_{n}-\mathcal{F}_{n}^{k}$ and into the interior of $\mathcal{P}_{n}$. [The last sentence, as well as the rest of the discussion leading to (3.30), would receive a trivial modification if $j_{n}=r_{G}$ (corresponding to constant $L_{h}$ over $\mathcal{P}_{n}$ ).] Therefore as $\beta \rightarrow 0$, the integrals in (3.24) localize around $j_{n}-\mathcal{F}_{n}^{k}$.

To further simplify the $n$th integral in (3.24), we now adopt a set of new coordinates - affinely related to $x_{i}$ and with unit Jacobian - that are convenient on $\mathcal{P}_{n}$. We pick a point on $j_{n}-\mathcal{F}_{n}^{k}$ as the new origin, and parameterize $j_{n}-\mathcal{F}_{n}^{k}$ with $\bar{x}_{1}, \ldots, \bar{x}_{j_{n}}$. We take $x_{\text {in }}$ to parameterize a direction perpendicular to all the $\bar{x} \mathrm{~s}$, and to increase as we go away from $j_{n}-\mathcal{F}_{n}^{k}$ and into the interior of $\mathcal{P}_{n}$. Finally, we pick $\tilde{x}_{1}, \ldots, \tilde{x}_{r_{G}-j_{n}-1}$ to parameterize the perpendicular directions to $x_{\text {in }}$ and the $\bar{x}$ s. Note that, because $\Delta L_{h}^{n}$ is linear on $\mathcal{P}_{n}$, it does not depend on the $\bar{x}$ s; they parameterize its flat directions. By re-scaling $\bar{x}, x_{\text {in }}, \tilde{x} \mapsto$ $\frac{\beta}{2 \pi} \bar{x}, \frac{\beta}{2 \pi} x_{\text {in }}, \frac{\beta}{2 \pi} \tilde{x}$, we can absorb the $\left(\frac{2 \pi}{\beta}\right)^{r_{G}}$ factors in (3.24) into the integrals, and write the $n$th resulting integral as

$$
\int_{\frac{2 \pi}{\beta} \mathcal{P}_{n}} \mathrm{~d}^{j_{n}} \bar{x} \mathrm{~d} x_{\text {in }} \mathrm{d}^{r_{G}-j_{n}-1} \tilde{x} e^{-2 \pi\left(\frac{b+b^{-1}}{2}\right) \Delta L_{h}^{n}\left(x_{\mathrm{in}}, \tilde{\mathbf{x}}\right)} .
$$

To eliminate $\beta$ from the exponent, we have used the fact that $\Delta L_{h}^{n}$ depends homogenously on the new coordinates. We are also denoting the re-scaled polytope schematically by $\frac{2 \pi}{\beta} \mathcal{P}_{n}$. Instead of integrating over all of $\frac{2 \pi}{\beta} \mathcal{P}_{n}$ though, we can restrict to $x_{\text {in }}<\epsilon / \beta$ with some (small) $\epsilon>0$. The reason is that the integrand of (3.25) is exponentially suppressed (as $\beta \rightarrow 0$ ) for $x_{\text {in }}>\epsilon / \beta$. We take $\epsilon>0$ to be small enough such that a hyperplane at $x_{\text {in }}=\epsilon / \beta$, and parallel to $j_{n}-\mathcal{F}_{n}^{k}$, cuts off a prismatoid $P_{\epsilon / \beta}^{n}$ from $\frac{2 \pi}{\beta} \mathcal{P}_{n}$. After restricting the integral in (3.25) to $P_{\epsilon / \beta}^{n}$, the integration over the $\bar{x} \mathrm{~s}$ is easy to perform. The only potential difficulty is that the range of the $\bar{x}$ coordinates may depend on $x_{\text {in }}$ and the $\tilde{x} s$. But since we are dealing with a prismatoid, the dependence is linear, and by the time the range is modified significantly (compared to its $O(1 / \beta)$ size on the re-scaled $j$-face $\frac{2 \pi}{\beta}\left(j_{n^{-}}\right.$ $\left.\mathcal{F}_{n}^{k}\right)$ ), the integrand is exponentially suppressed. Therefore we can neglect the dependence of the range of the $\bar{x}$ s on the other coordinates in (3.25). The integral then simplifies to

$$
\left(\frac{2 \pi}{\beta}\right)^{j_{n}} \operatorname{vol}\left(j_{n}-\mathcal{F}_{n}^{k}\right) \int_{\hat{P}_{\epsilon / \beta}^{n}} \mathrm{~d} x_{\mathrm{in}} \mathrm{d}^{r_{G}-j_{n}-1} \tilde{x} e^{-2 \pi\left(\frac{b+b^{-1}}{2}\right) \Delta L_{h}^{n}\left(x_{\mathrm{in}}, \tilde{\mathbf{x}}\right)},
$$

where $\hat{P}_{\epsilon / \beta}^{n}$ is the pyramid obtained by restricting $P_{\epsilon / \beta}^{n}$ to $\bar{x}_{1}=\ldots=\bar{x}_{j_{n}}=0$.

We now take $\epsilon \rightarrow \infty$ in (3.26). This introduces exponentially small error, as the integrand is exponentially suppressed (as $\beta \rightarrow 0$ ) for $x_{\text {in }}>\epsilon / \beta$. The resulting integral is strictly positive, because it is the integral of a strictly positive function. We denote by $I_{n}$ the result of the integral multiplied by $\operatorname{vol}\left(j_{n}-\mathcal{F}_{n}^{k}\right)$. Putting everything together, we can simplify (3.24) as

$$
Z^{\mathrm{SUSY}}(b, \beta) \approx \sum_{n} e^{-\mathcal{E}_{0}^{D K}(b, \beta)-\frac{4 \pi^{2}}{\beta}\left(\frac{b+b^{-1}}{2}\right) L_{h \min }^{n}}\left(\frac{2 \pi}{\beta}\right)^{j_{n}} I_{n} .
$$

The dominant contribution comes, of course, from the terms/polytopes whose $L_{h \text { min }}^{n}$ are smallest. If these terms are labeled by $n=n_{*}^{1}, n_{*}^{2}, \ldots$, what we referred to as $\mathfrak{h}_{q u}$ and 
$\operatorname{dimh} \mathfrak{h}_{q u}$ above can now be precisely defined via

$$
\mathfrak{h}_{q u}:=\bigcup_{n_{*}} j_{n_{*}}-\mathcal{F}_{n_{*}}^{k}, \quad \operatorname{dimh} \mathfrak{h}_{q u}:=\max \left(j_{n_{*}}\right) .
$$

Put colloquially, if $\mathfrak{h}_{q u}$ has multiple connected components, by dim $\mathfrak{h}_{q u}$ we mean the dimension of the component(s) with greatest dimension, while if a connected component consists of several intersecting flat elements inside $\mathfrak{h}_{c l}$, by its dimension we mean the dimension of the flat element(s) of maximal dimension.

The final result for the high-temperature asymptotics of $Z^{\mathrm{SUSY}}(b, \beta)$ is then

$$
Z^{\mathrm{SUSY}}(b, \beta) \approx e^{-\mathcal{E}_{0}^{D K}(b, \beta)-\frac{4 \pi^{2}}{\beta}\left(\frac{b+b^{-1}}{2}\right) L_{h} \min }\left(\frac{2 \pi}{\beta}\right)^{\operatorname{dimh}_{q u}}
$$

where $L_{h \text { min }}:=L_{h \text { min }}^{n_{*}}$.

Using the explicit expression (3.10) for $\mathcal{E}_{0}^{D K}(b, \beta)$, and noting that (3.29) is accurate up to a multiplicative factor of order $\beta^{0}$, we arrive at

$$
\begin{array}{r}
\ln Z^{\mathrm{SUSY}}(b, \beta)=-\frac{\pi^{2}}{3 \beta}\left(\frac{b+b^{-1}}{2}\right)\left(\operatorname{Tr} R+12 L_{h \min }\right)+\operatorname{dim} \mathfrak{h}_{q u} \ln \left(\frac{2 \pi}{\beta}\right)+O\left(\beta^{0}\right) \\
\left(\text { when } Q_{h}=0\right) .
\end{array}
$$

As the last step in deriving (3.30), we now outline the argument justifying, when $Q_{h}=0$, our use of the estimate $(2.14)$ on $\mathcal{S}_{g}^{(\beta)}$. First, an analysis similar to the one that took us from (3.21) to (3.30), shows that (3.30) is not modified if the region $\mathcal{S}_{g}^{(\beta)}$ is excised from the integral (3.21) (in particular, after excising $\mathcal{S}_{g}^{(\beta)}$, since $L_{h}$ is continuous and piecewise linear, $L_{h}$ min moves up by an $O(\beta)$ amount, leaving (3.30) unchanged). The effect of the integral over $\mathcal{S}_{g}^{(\beta)}$ is thus negligible (additive $O\left(\beta^{0}\right)$ ) on the asymptotics (3.30). On the other hand, the estimate (2.15) guarantees that the actual contribution to $Z^{\mathrm{SUSY}}(b, \beta)$ coming from $\mathcal{S}_{g}^{(\beta)}$ is of the same order as the integral we just found negligible. Thus our previously unjustified use of $(2.14)$ on $\mathcal{S}_{g}^{(\beta)}$ introduces a negligible $\left(O\left(\beta^{0}\right)\right)$ error in (3.30).

Let us now discuss the obstruction to performing a similar analysis for theories with nonzero $Q_{h}$, and hence nonzero phase $\Theta$. For such theories (3.24) would apply, except that the exponent of the integrand would contain an $i \Theta$. However, the resulting integrals can not be written in a form similar to (3.25) in any obvious way. In subsections 3.1 and 3.2 below, we consider special cases with nonzero $Q_{h}$, in which this obstruction can be bypassed.

In the remaining of this section we consider several specific examples. We will have the following two goals in mind:

- Deriving more precise asymptotics than (3.30). We will find that improving (3.30) to include the $O\left(\beta^{0}\right)$ term is generally straightforward, but further obtaining the $O(\beta)$ term is difficult for theories with $\operatorname{dimh} \mathfrak{h}_{q u}>0$. The only example with $\operatorname{dimh} \mathfrak{h}_{q u}>0$ for which we will improve (3.30) to $O(\beta)$ - and in fact to all-orders - accuracy is the $\mathrm{SO}(3) \mathrm{SQCD}$ with two flavors in the special case $b=1$. What enables us to obtain such a precise result for this theory is a remarkable equality between its SUSY 
partition function and the Schur partition function of the $\mathrm{SU}(2) \mathcal{N}=4 \mathrm{SYM}$. The latter is known to coincide with the partition function of a free-fermion system on a circle [7], and is thus exceptionally well under control. The Schur partition function of the $\mathrm{SU}(N) \mathcal{N}=4 \mathrm{SYM}$ is studied in appendix B.

- Analyzing cases with nonzero $\mathbf{Q}_{\mathbf{h}}$ (and hence nonzero $\Theta$ ). If $\mathbf{x}=0$ is the unique minimum of the Rains function, nonzero $Q_{h}$ can in fact be easily accommodated, and as we will show in subsection 3.1 the relation (3.30) remains valid. A theory exemplifying this scenario is the magnetic Pouliot theory [50] with $N_{f}=7$. When the Rains function is minimized away from the origin, or when $\operatorname{dimh} \mathfrak{h}_{q u}>0$, nonzero $Q_{h}$ makes it difficult (as already mentioned above) to obtain precise general statements similar to (3.30). The $\mathbb{Z}_{3}$ orbifold theory studied in subsection 3.2 below, exemplifies the scenario with $Q_{h} \neq 0$ and $\operatorname{dimh} \mathfrak{h}_{q u}>0$. However, it appears that $Q_{h}$ vanishes on $\mathfrak{h}_{q u}$ in that case, suggesting that (3.30) is not modified for the $\mathbb{Z}_{3}$ orbifold theory either.

\section{$3.1 \quad Z_{S^{3}}$ finite}

Consider the cases where $\mathbf{x}=0$ is the unique minimum of the Rains function $L_{h}(\mathbf{x})$; in particular, it is isolated. Then it follows from (3.9) that the matrix-integral of $Z^{\text {SUSY }}$ localizes around $\mathbf{x}=0$, and receives exponentially small contribution from everywhere else. (Note that when $\Theta \neq 0$, to obtain the asymptotics of (3.9) one must in general first resolve the tension between minimizing $V^{\text {eff }}$ and making $\Theta$ stationary. But, firstly, since according to eq. (3.17) $Q_{h}$ is stationary at the origin, we have the best of both worlds in the present section, and need not worry about stationarity of the phase $\Theta$. Secondly, in deriving (3.36) we will not even use the stationarity of $Q_{h}$ below; the result in (3.36) then justifies focusing on a neighborhood of the origin, as the positivity of the Rains function everywhere else guarantees that the correction to (3.36) coming from the rest of $\mathfrak{h}_{c l}$ is exponentially suppressed.) We can thus restrict the domain of integration in (3.2) to a small neighborhood of $\mathbf{x}=0$, say a hypercube $\mathfrak{h}_{c l}^{\epsilon}$ defined by $\left|x_{i}\right|<\epsilon$, in which the central estimates (2.16) and (2.18) apply. [In asymptotic analysis, the procedure of cutting down the range of integration to some manageable size is sometimes called tails pruning.] Using the central estimates (2.16) and (2.18) for every elliptic gamma function inside the integrand of (3.2), we obtain a product of several $e^{2 \pi i R_{0}}$ factors the result of which we denote by $e^{2 \pi i R_{0}^{\text {integrand }}(\mathbf{x} ; b, \beta)}$, and also one hyperbolic gamma function for each elliptic gamma function. On the other hand, according to (2.9), we have the following estimate for the Pochhammer symbols in the prefactor of (3.2):

$$
(p ; p)^{r_{G}}(q ; q)^{r_{G}} \simeq e^{2 \pi i \cdot r_{G} \cdot R_{0}^{\mathrm{U}(1)}(b, \beta)} \times\left(\frac{2 \pi}{\beta}\right)^{r_{G}}, \quad(\text { as } \beta \rightarrow 0)
$$

where we have defined

$$
e^{2 \pi i R_{0}^{\mathrm{U}(1)}(b, \beta)}:=e^{-\pi^{2}\left(b+b^{-1}\right) / 6 \beta} e^{\left(b+b^{-1}\right) \beta / 24} .
$$

We now define $e^{2 \pi i R_{0}^{\text {total }}}$ as follows

$$
e^{2 \pi i R_{0}^{\mathrm{total}}(\mathbf{x} ; b, \beta)}:=e^{2 \pi i \cdot r_{G} \cdot R_{0}^{\mathrm{U}(1)}(b, \beta)} \cdot e^{2 \pi i R_{0}^{\mathrm{integrand}}(\mathbf{x} ; b, \beta)} .
$$


The small- $\beta$ asymptotics of the partition function (3.1) can then be written as

$$
Z^{\operatorname{SUSY}}(b, \beta) \simeq e^{-\beta E_{\text {susy }}(b)} \frac{\left(\frac{2 \pi}{\beta}\right)^{r_{G}}}{|W|} \int_{\mathfrak{h}_{c l}^{\epsilon}} \mathrm{d}^{r_{G}} x e^{2 \pi i R_{0}^{\mathrm{total}}(\mathbf{x} ; b, \beta)} \frac{\prod_{\chi} \prod_{\rho^{\chi \in \Delta_{\chi}}} \Gamma_{h}\left(r_{\chi} \omega+\frac{2 \pi}{\beta}\left\langle\rho^{\chi} \cdot \mathbf{x}\right\rangle\right)}{\prod_{\alpha_{+}} \Gamma_{h}\left( \pm \frac{2 \pi}{\beta}\left\langle\alpha_{+} \cdot \mathbf{x}\right\rangle\right)}
$$

Since the function $R_{0}(x ; \sigma, \tau)$ defined in (2.17) is a cubic polynomial in $x$, one might expect $R_{0}^{\text {total }}(\mathbf{x} ; b, \beta)$ to be also cubic in $x_{i}$. But because of the gauge-gauge-gauge anomaly cancelation, the cubic terms in $R_{0}^{\text {total }}$ cancel. Because of the vanishing of the ABJ anomaly for the $\mathrm{U}(1)_{R}$ current, the quadratic terms in $R_{0}^{\text {total }}$ also cancel. In fact $R_{0}^{\text {total }}$ is completely $x_{i}$-independent, because the linear terms in it also cancel due to the vanishing of the mixed gauge- $\mathrm{U}(1)_{R}^{2}$ and gauge-gravity-gravity anomalies (see the related discussion in section 5 of [10]). The end result is

$$
e^{2 \pi i R_{0}^{\text {total }}(\mathbf{x} ; b, \beta)}=e^{-\mathcal{E}_{0}^{D K}(b, \beta)+\beta E_{\text {susy }}(b)} .
$$

Using the above simplification for $R_{0}^{\text {total }}$, and re-scaling the integration variables in (3.34) via $x \mapsto\left(\frac{2 \pi}{\beta}\right) x$, we arrive at

$$
Z^{\mathrm{SUSY}}(b, \beta) \simeq e^{-\mathcal{E}_{0}^{D K}(b, \beta)} Z_{S^{3}}(b ; 2 \pi \epsilon / \beta), \quad(\text { as } \beta \rightarrow 0)
$$

where

$$
Z_{S^{3}}(b ; \Lambda):=\frac{1}{|W|} \int_{\Lambda} \mathrm{d}^{r_{G}} x \frac{\prod_{\chi} \prod_{\rho^{\chi} \in \Delta_{\chi}} \Gamma_{h}\left(r_{\chi} \omega+\left\langle\rho^{\chi} \cdot \mathbf{x}\right\rangle\right)}{\prod_{\alpha_{+}} \Gamma_{h}\left( \pm\left\langle\alpha_{+} \cdot \mathbf{x}\right\rangle\right)},
$$

is the matrix-integral computing the squashed-three-sphere partition function of the reduced theory on $S_{\beta}^{1}$ (cf. eq. (5.23) of [35]), assuming the same R-charge assignments as those directly descending from the parent $4 \mathrm{~d}$ theory. We are keeping the cut-off $\Lambda$ explicit, emphasizing that the integration is over the hypercube $\left|x_{i}\right|<\Lambda$.

The r.h.s. of (3.36) still has an intricate temperature-dependence through the $\beta$ dependent cut-off for $Z_{S^{3}}$. Our final step in analyzing the high-temperature asymptotics of $Z^{\text {SUSY }}$ for theories with finite $Z_{S^{3}}$ is to argue that taking $\epsilon \rightarrow \infty$ in (3.36) introduces exponentially small error. [In asymptotic analysis, the procedure of extending the range of integration to an infinitely large set, over which computations are simplified, is sometimes called tails completion.] Upon using the asymptotics of the hyperbolic gamma function in (2.19), we find that the integrand of $Z_{S^{3}}(b ; \Lambda)$ can be estimated, as $x \rightarrow \infty$, by

$$
\frac{\prod_{\chi} \prod_{\rho^{\chi} \in \Delta_{\chi}} \Gamma_{h}\left(r_{\chi} \omega+\left\langle\rho^{\chi} \cdot \mathbf{x}\right\rangle\right)}{\prod_{\alpha_{+}} \Gamma_{h}\left( \pm\left\langle\alpha_{+} \cdot \mathbf{x}\right\rangle\right)} \approx e^{-2 \pi\left(\frac{b+b^{-1}}{2}\right) \tilde{L}_{S^{3}}(\mathbf{x})+2 \pi i \tilde{Q}_{S^{3}}(\mathbf{x})},
$$

with $\tilde{L}_{S^{3}}$ and $\tilde{Q}_{S^{3}}$ the functions defined in (3.20) and (3.18). Our assumption that $\mathbf{x}=0$ is an isolated minimum of $L_{h}(\mathbf{x})$ now implies that for $\mathbf{x}$ small enough, $\tilde{L}_{S^{3}}(\mathbf{x})$ is strictly positive. But since $\tilde{L}_{S^{3}}(\mathbf{x})$ is a homogenous function of $\mathbf{x}$, its strict positivity for small enough $\mathbf{x}$ implies its strict positivity for all $x$. As a result, for $x_{i} \propto \Lambda$ the integrand of $Z_{S^{3}}(b ; \Lambda)$ is exponentially small as $\Lambda \rightarrow \infty$, and tails completion introduces an error that 
is exponentially small in the cut-off. Thus taking $\epsilon \rightarrow \infty$ in (3.36) introduces an error of the type $e^{-\epsilon / \beta}$, and we can write

$$
\ln Z^{\mathrm{SUSY}}(b, \beta) \sim-\mathcal{E}_{0}^{D K}(b, \beta)+\ln Z_{S^{3}}(b), \quad(\text { as } \beta \rightarrow 0)
$$

where $Z_{S^{3}}(b):=Z_{S^{3}}(b ; \infty)$ is the squashed-three-sphere partition function with the cut-off removed. The symbol $\sim$ indicates that the error is beyond all-orders, but our arguments above imply the stronger result that the error is exponentially small, of the type $e^{-1 / \beta}$.

We have demonstrated that if $\mathbf{x}=0$ is the unique global minimum of the Rains function, then $Z_{S^{3}}(b)$ is finite and (3.39) holds. Our arguments show in fact that if $\mathbf{x}=0$ is an isolated local minimum of $L_{h}$, then $Z_{S^{3}}(b)$ is finite (although (3.39) does not hold if $\mathbf{x}=0$ is not a global minimum). Conversely, if $Z_{S^{3}}(b)$ is finite, then $\mathbf{x}=0$ is an isolated local minimum of the Rains function. This is because for $Z_{S^{3}}(b)$ to be finite, its integrand in (3.37) must decay at large $\mathbf{x}$. Hence $\tilde{L}_{S^{3}}(\mathbf{x})$ must be positive for large $\mathbf{x}$, and because of its homogeneity, also for small nonzero $\mathbf{x}$. But for small $\mathbf{x}$ the two functions $\tilde{L}_{S^{3}}$ and $L_{h}$ coincide. Therefore $L_{h}$ is strictly positive for small enough but nonzero $\mathbf{x}$. Since $L_{h}(\mathbf{x}=0)=0$, the desired result follows.

Combining (3.39) and (3.1), we obtain

$$
\ln \mathcal{I}(b, \beta) \sim-\mathcal{E}_{0}^{D K}(b, \beta)+\ln Z_{S^{3}}(b)+\beta E_{\text {susy }}(b) \quad(\text { as } \beta \rightarrow 0) .
$$

A relation similar to (3.40) holds for an equivariant generalization of $\mathcal{I}(b, \beta)$, which we denote by $\mathcal{I}\left(b, \beta ; m_{a}\right)$. The latter contains fugacities $u_{a}=e^{i \beta m_{a}}$ associated to conserved $\mathrm{U}(1)_{a}$ charges of the theory (the U(1)s may reside in the Cartan torus of a non-abelian group); setting all $u_{a}$ to 1 , we recover $\mathcal{I}(b, \beta)$. In that generalized relation, $E_{\text {susy }}(b)$ is replaced with $E_{\text {susy }}\left(b ; m_{a}\right)$. Therefore all the 't Hooft anomalies of a SUSY gauge theory with a U(1) R-symmetry, with a semi-simple gauge group, and with a Rains function that is minimized only at the origin, can be extracted from the high-temperature asymptotics of the equivariant Romelsberger index of the theory. This statement is related (but not equivalent) to some of the claims in [31], which were made there in the context of SU(N) SQCD.

\subsection{1 $A_{k}$ SQCD theories with $N_{f}>\frac{2 N}{k+1}$}

Take now the example of $A_{k} \mathrm{SQCD}$ with $\mathrm{SU}(N)$ gauge group. This theory has a chiral multiplet with R-charge $r_{a}=\frac{2}{k+1}$ in the adjoint, $N_{f}$ flavors in the fundamental with Rcharge $r_{f}=1-\frac{2}{k+1} \frac{N}{N_{f}}$, and $N_{f}$ flavors in the anti-fundamental with R-charge $r_{\bar{f}}=r_{f}$. For $r_{f}$ to be well-defined we must have $N_{f}>2 N /(k+1)$.

We will not bother commenting on the IR phase of the theory on flat space for various ranges of parameters. What matters for us is that the supersymmetric partition function of the theory on $S_{b}^{3} \times S_{\beta}^{1}$ is well-defined if $N_{f}>2 N /(k+1)$.

The SUSY partition function is (cf. [11])

$$
\begin{aligned}
Z_{A_{k}}^{\mathrm{SUSY}}(b, \beta)=e^{-\beta E_{\text {susy }}(b)} & \frac{(p ; p)^{N-1}(q ; q)^{N-1}}{N !} \Gamma^{N-1}\left((p q)^{r_{a} / 2}\right) \int \mathrm{d}^{N-1} x \\
& \left(\prod_{1 \leq i<j \leq N} \frac{\Gamma\left((p q)^{r_{a} / 2}\left(z_{i} / z_{j}\right)^{ \pm 1}\right)}{\Gamma\left(\left(z_{i} / z_{j}\right)^{ \pm 1}\right)}\right) \prod_{i=1}^{N} \Gamma^{N_{f}}\left((p q)^{r_{f} / 2} z_{i}^{ \pm 1}\right),
\end{aligned}
$$

with $\prod_{i=1}^{N} z_{i}=1$. 


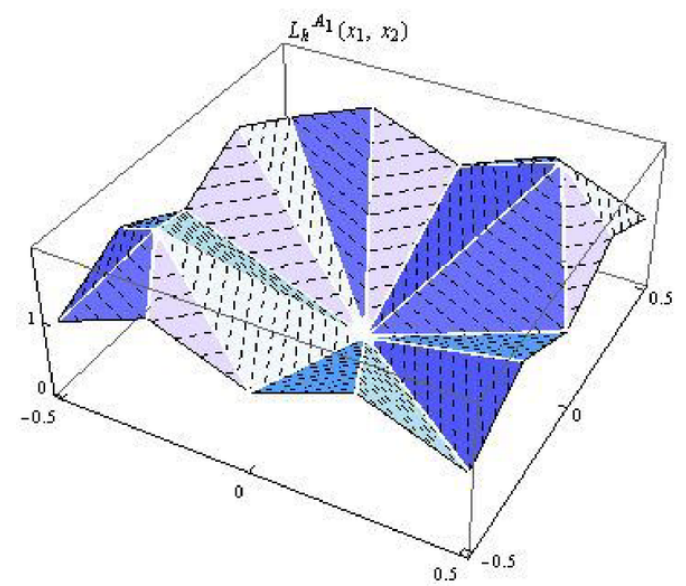

Figure 2. The Rains function of the $A_{1} \mathrm{SU}(3)$ theory - also known as SU(3) SQCD - for $N_{f}>3$. Note that the minimum lies at $x_{1}=x_{2}=0$.

The Rains function of the theory is

$$
\begin{aligned}
L_{h}^{A_{k}}\left(x_{1}, \ldots, x_{N-1}\right) & =N_{f}\left(1-r_{f}\right) \sum_{i=1}^{N} \vartheta\left(x_{i}\right)+\left(1-r_{a}\right) \sum_{1 \leq i<j \leq N} \vartheta\left(x_{i}-x_{j}\right)-\sum_{1 \leq i<j \leq N} \vartheta\left(x_{i}-x_{j}\right) \\
& =\frac{2}{k+1}\left(N \sum_{i} \vartheta\left(x_{i}\right)-\sum_{1 \leq i<j \leq N} \vartheta\left(x_{i}-x_{j}\right)\right) .
\end{aligned}
$$

The $x_{N}$ in the above expression is constrained by $\sum_{i=1}^{N} x_{i} \in \mathbb{Z}$, although since $\vartheta(x)$ is periodic with period one we can simply replace $x_{N} \rightarrow-x_{1}-\cdots-x_{N-1}$. For $k=1$ and $N=3$, the resulting function is illustrated in figure 2 .

We recommend that the reader convince herself that the Rains function in (3.42) can be easily written down by examining the integrand of (3.41). Whenever the index (or the SUSY partition function) of a theory is available in the literature, a similar examination of the integrand quickly yields the theory's $L_{h}$ and $Q_{h}$ functions.

Using Rains's generalized triangle inequality (2.20), in the special case where $d_{i}=0$, we find that the above function is minimized when all $x_{i}$ are zero. This establishes that the integrand of (3.41) is localized around $x_{i}=0$, and is exponentially suppressed everywhere else, as $\beta \rightarrow 0$. The asymptotic relation (3.39) then applies with

$$
Z_{S^{3}}^{A_{k}}(b)=\frac{\Gamma_{h}^{N-1}\left(r_{a} \omega\right)}{N !} \int \mathrm{d}^{N-1} x\left(\prod_{1 \leq i<j \leq N} \frac{\Gamma_{h}\left(r_{a} \omega \pm\left(x_{i}-x_{j}\right)\right)}{\Gamma_{h}\left( \pm\left(x_{i}-x_{j}\right)\right)}\right) \prod_{i=1}^{N} \Gamma_{h}^{N_{f}}\left(r_{f} \omega \pm x_{i}\right) .
$$

The convergence of the above integral (over $\left.x_{1}, \ldots, x_{N-1} \in\right]-\infty, \infty[$ ) follows from our general discussion above (3.39), but it can also be explicitly verified using the estimate (2.19) and the generalized triangle inequality (2.23).

A similar story applies to the $D$ and $E$ type $\mathrm{SU}(N)$ SQCD theories [25], and also to the $\operatorname{Sp}(2 N)$ SQCD theories. We leave it as an exercise for the interested reader to reproduce 


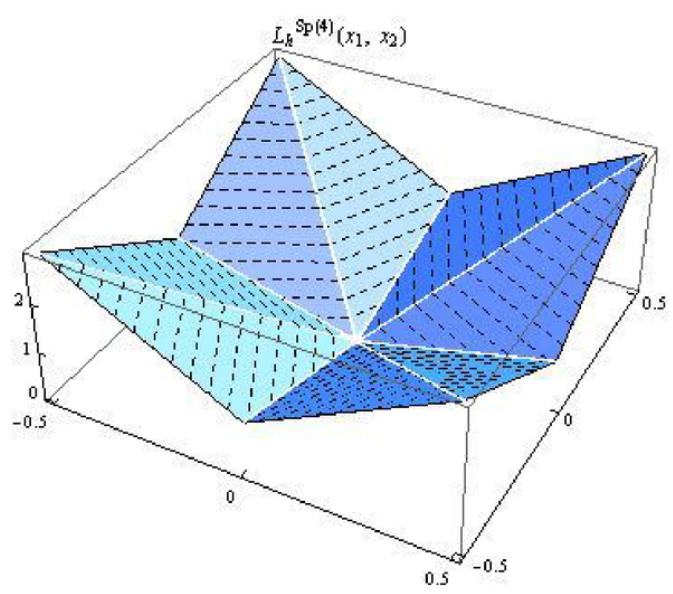

Figure 3. The Rains function of the $\operatorname{Sp}(4) \operatorname{SQCD}$ theory for $N_{f}>3$. Note that the minimum lies at $x_{1}=x_{2}=0$.

the plot of the Rains function of the $\operatorname{Sp}(4) \mathrm{SQCD}$ for $N_{f}>3$ shown in figure 3. (The Romelsberger index of the $\operatorname{Sp}(2 N)$ SQCD theories can be found in [11]. Lemma 3.3 of Rains [13] establishes that $L_{h}^{\mathrm{Sp}(2 N)}(\mathbf{x})$ is minimized only at $\mathbf{x}=0$, for any $N_{f}>N+1$.)

\subsubsection{The magnetic Pouliot theory with $N_{f}=7$}

We now consider a chiral theory with $Q_{h} \neq 0$. This is the $\mathrm{SU}(3)$ theory [50] with seven chiral multiplets of R-charge $r_{\bar{f}}=13 / 21$ in the anti-fundamental representation, a chiral multiplet of R-charge $r_{w}=2 / 3$ in the symmetric tensor representation, and 28 chiral gauge-singlets with R-charge $r_{M}=4 / 7$.

The SUSY partition function is (cf. [23])

$$
\begin{aligned}
Z_{P_{m 7}}^{\mathrm{SUSY}}(b, \beta)=e^{-\beta E_{\text {susy }}(b)} & \frac{(p ; p)^{2}(q ; q)^{2}}{3 !} \Gamma^{28}\left((p q)^{r_{M} / 2}\right) \int \mathrm{d}^{2} x \\
& \left(\prod_{1 \leq i<j \leq 3} \frac{\Gamma\left((p q)^{r_{w} / 2} z_{i} z_{j}\right)}{\Gamma\left(\left(z_{i} / z_{j}\right)^{ \pm 1}\right)}\right) \prod_{i=1}^{3} \Gamma\left((p q)^{r_{w} / 2} z_{i}^{2}\right) \Gamma^{7}\left((p q)^{r_{\bar{f}} / 2} z_{i}^{-1}\right),
\end{aligned}
$$

with $\prod_{i=1}^{3} z_{i}=1$.

The Rains function of the theory is

$$
\begin{aligned}
L_{h}^{P_{m 7}\left(x_{1}, x_{2}\right)=} & \frac{1}{2}\left(1-r_{w}\right) \sum_{1 \leq i<j \leq 3} \vartheta\left(x_{i}+x_{j}\right)+\frac{1}{2}\left(1-r_{w}\right) \sum_{i=1}^{3} \vartheta\left(2 x_{i}\right) \\
& +7 \cdot \frac{1}{2}\left(1-r_{\bar{f}}\right) \sum_{i=1}^{3} \vartheta\left(x_{i}\right)-\sum_{1 \leq i<j \leq 3} \vartheta\left(x_{i}-x_{j}\right) \\
= & \frac{1}{6} \sum_{1 \leq i<j \leq 3} \vartheta\left(x_{i}+x_{j}\right)+\frac{1}{6} \sum_{i=1}^{3} \vartheta\left(2 x_{i}\right)+\frac{13}{6} \sum_{i=1}^{3} \vartheta\left(x_{i}\right)-\sum_{1 \leq i<j \leq 3} \vartheta\left(x_{i}-x_{j}\right),
\end{aligned}
$$

with $x_{3}=-x_{1}-x_{2}$. This function is illustrated in figure 4 . 


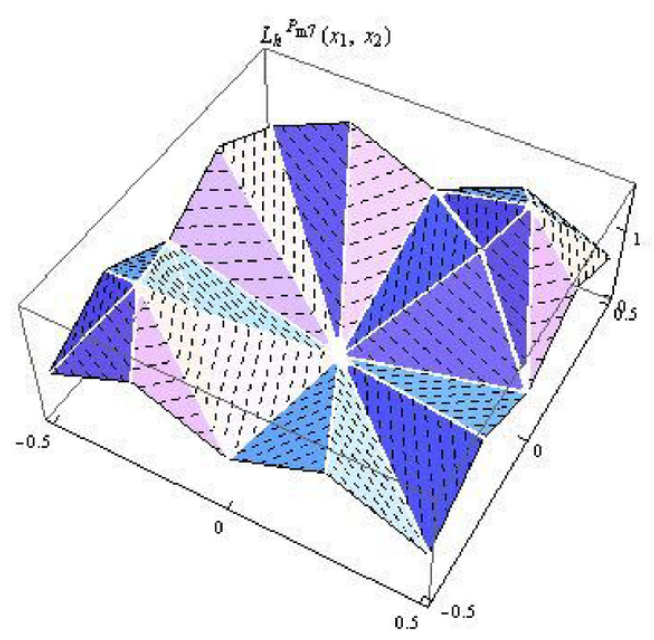

Figure 4. The Rains function of the $\mathrm{SU}(3)$ magnetic Pouliot theory with $N_{f}=7$. Note that the minimum lies at $x_{1}=x_{2}=0$.

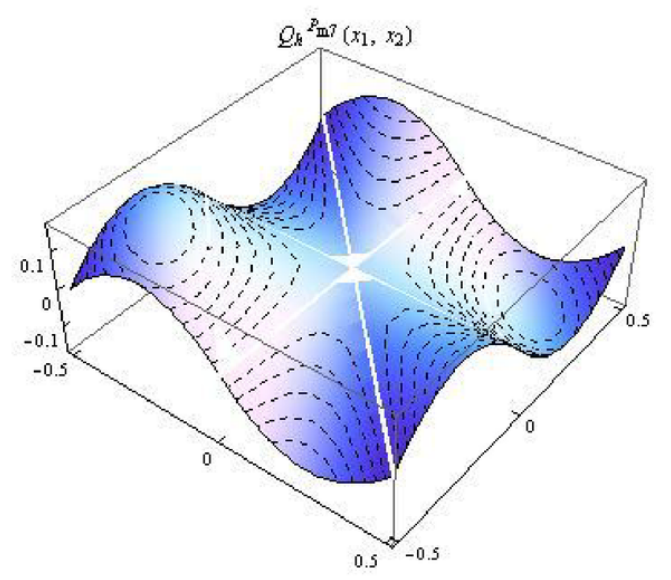

Figure 5. The $Q_{h}$ function of the $\mathrm{SU}(3)$ magnetic Pouliot theory with $N_{f}=7$. Note the stationarity at $x_{1}=x_{2}=0$.

For this theory $Q_{h}$ is given by

$$
Q_{h}^{P_{m 7}}\left(x_{1}, x_{2}\right)=\frac{1}{12} \sum_{1 \leq i<j \leq 3} \kappa\left(x_{i}+x_{j}\right)+\frac{1}{12} \sum_{i=1}^{3} \kappa\left(2 x_{i}\right)+7 \cdot \frac{1}{12} \sum_{i=1}^{3} \kappa\left(-x_{i}\right),
$$

again with $x_{3}=-x_{1}-x_{2}$. This function is illustrated in figure 5 .

As figure 4 demonstrates, the Rains function has a unique minimum at the origin of $\mathfrak{h}_{c l}$. Therefore the asymptotics (3.39) applies, with some $Z_{S^{3}}(b)$ whose derivation we leave to the interested reader.

\section{2 $Z_{S^{3}}$ power-law divergent (or: the effect of an unlifted Coulomb branch)}

Assume now that $\mathbf{x}=0$ is a global minimum of $L_{h}$, and that the zero-set of $L_{h}$ is a connected subset of $\mathfrak{h}_{c l}$, which we refer to as $\mathfrak{h}_{q u}$. In such cases the Rains function has "flat 
directions" along $\mathfrak{h}_{q u}$. These flat directions present an obstruction to the tails pruning of the previous subsection. Therefore we can not obtain asymptotic expressions as precise as (3.39). Nonzero $Q_{h}$ would present another difficulty in the general analysis. We thus assume for now that $Q_{h}=0$; the $\mathbb{Z}_{3}$ orbifold studied below provides an example with $Q_{h} \neq 0$. Equation (3.30) then reads

$$
\ln Z^{\mathrm{SUSY}}(b, \beta)=-\mathcal{E}_{0}^{D K}(b, \beta)+\operatorname{dim} \mathfrak{h}_{q u} \cdot \ln \left(\frac{2 \pi}{\beta}\right)+O(1) \quad(\text { as } \beta \rightarrow 0) .
$$

We will derive more precise asymptotic expressions in our detailed case-studies below. But before that, some comments on the relation between the logarithmic term in (3.47) and the "unlifted Coulomb branches" are in order.

The SUSY partition function is computed by a path-integral on $S_{b}^{3} \times S_{\beta}^{1}$. If the theory on $S_{b}^{3} \times S_{\beta}^{1}$ contains unlifted (or quantum) zero-modes with a compact target manifold, the path-integral receives a multiplicative contribution from the volume of the target space. The parameters $x_{i}$ that we have used above, are related via $\sigma_{i}=\frac{2 \pi}{\beta} x_{i}$ to the scalar zeromodes $\sigma_{i}$ associated with the holonomies (cf. section 2 of [35]). Since $x_{i}$ are periodic with period one, the volume of the target space of the $\sigma_{i}$ is proportional to $\left(\frac{2 \pi}{\beta}\right)^{r_{G}}$; we say proportional, because one must mod out the product space by the large gauge transformations associated to the Weyl group of $G$; this introduces an $O(1)$ factor though, and can be neglected for our current discussion. Not all of the $r_{G}$ scalars $\sigma_{i}$ are quantum zero modes; some of them are lifted by quantum mechanically generated potentials. We interpret $V^{\text {eff }}$ as (the high-temperature asymptotics of) such a potential, and thus conclude that the unlifted zero modes are those $\sigma_{i}$ that correspond to the $x_{i}$ parameterizing $\mathfrak{h}_{q u}$. Because the target space of these unlifted $\sigma_{i}$ decompactifies as $\beta \rightarrow 0$, we say that theories with $\operatorname{dimh} \mathfrak{h}_{q u}>0$ experience Coulomb branch decompactification at high temperatures on $S_{b}^{3} \times S_{\beta}^{1}$. The word "Coulomb branch" is used because the $\sigma_{i}$ parameterize (part of) the Coulomb branch of the $3 \mathrm{~d} \mathcal{N}=2$ gauge theory obtained by reducing the $4 \mathrm{~d} \mathcal{N}=1$ gauge theory on $S_{\beta}^{1}$.

With the same assumptions that $\mathbf{x}=0$ is a global minimum of $L_{h}$ and that the zero-set of $L_{h}$ is a connected subspace of $\mathfrak{h}_{c l}$ denoted $\mathfrak{h}_{q u}$, we can demonstrate that $Z_{S^{3}}(b ; \Lambda)$ defined in (3.37) must be power-law divergent in $\Lambda$. Combining (3.37) and (3.38) we can find the leading $\Lambda$-dependence of $Z_{S^{3}}(b ; \Lambda)$ from

$$
Z_{S^{3}}(b ; \Lambda) \approx \frac{1}{|W|} \int_{\Lambda} \mathrm{d}^{r} x e^{-2 \pi\left(\frac{b+b^{-1}}{2}\right) \tilde{L}_{S^{3}}(\mathbf{x})} .
$$

Now since $L_{h}$ has flat directions, so does $\tilde{L}_{S^{3}}$. Assume that the flat directions of $\tilde{L}_{S^{3}}$ parameterize a space of dimension $\operatorname{dim} \mathfrak{h}_{q u}$ (this is the case in the $\mathrm{SO}(2 N+1)$ SQCD and the $\mathcal{N}=4 \mathrm{SYM}$ examples below, and we conjecture that it is the case also in the $\mathbb{Z}_{2}$ and $\mathbb{Z}_{3}$ orbifold theories; more generally, we suspect — but have not been able to show - that whenever $L_{h \text { min }}=0$, the flat directions of $\tilde{L}_{S^{3}}$ parameterize a space of dimension $\operatorname{dimh} \mathfrak{h}_{q u}$ ). Along these flat directions the integrand of $Z_{S^{3}}(b ; \Lambda)$ does not decay at large $\Lambda$, and thus upon taking the cut-off to infinity $Z_{S^{3}}(b ; \Lambda)$ diverges as $\Lambda^{\text {dimf }}{ }_{q u}$. Such power-law divergences are expected for $3 \mathrm{~d}$ theories with an unlifted Coulomb branch [8]. 


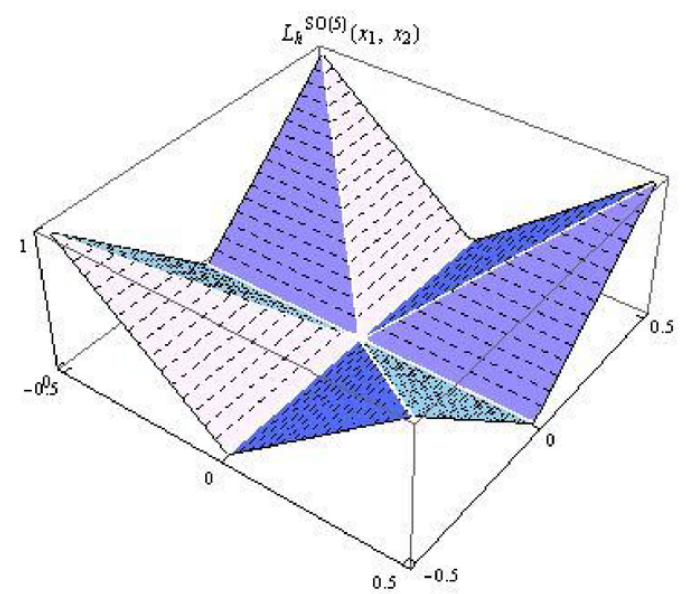

Figure 6. The Rains function of the SO(5) SQCD theory with $N_{f}>3$. Note the flat directions along the axes.

\subsection{1 $\mathrm{SO}(2 N+1)$ SQCD with $N_{f}>2 N-1$}

Consider the $\mathrm{SO}(n) \mathrm{SQCD}$ theories with $N_{f}$ chiral matter multiplets of R-charge $r=1-\frac{n-2}{N_{f}}$ in the vector representation. For the R-charges to be greater than zero, and the gauge group to be semi-simple, we must have $0<n-2<N_{f}$.

We perform the analysis for odd $n$. The analysis for even $n$ is completely analogous, and the result is similar. The SUSY partition function of $\mathrm{SO}(2 N+1)$ SQCD is given by (cf. [11])

$$
\begin{aligned}
Z_{\mathrm{SO}(2 N+1)}^{\mathrm{SUSY}}(b, \beta)= & e^{-\beta E_{\text {susy }}(b)} \frac{(p ; p)^{N}(q ; q)^{N}}{2^{N} N !} \Gamma^{N_{f}}\left((p q)^{r / 2}\right) \\
& \times \int \mathrm{d}^{N} x \frac{\prod_{j=1}^{N} \Gamma^{N_{f}}\left((p q)^{r / 2} z_{j}^{ \pm 1}\right)}{\prod_{j=1}^{N} \Gamma\left(z_{j}^{ \pm 1}\right) \prod_{i<j}\left(\Gamma\left(\left(z_{i} z_{j}\right)^{ \pm 1}\right) \Gamma\left(\left(z_{i} / z_{j}\right)^{ \pm 1}\right)\right)} .
\end{aligned}
$$

The Rains function of the theory is

$$
L_{h}^{\mathrm{SO}(2 N+1)}(\mathbf{x})=(2 N-2) \sum_{j=1}^{N} \vartheta\left(x_{j}\right)-\sum_{1 \leq i<j \leq N} \vartheta\left(x_{i}+x_{j}\right)-\sum_{1 \leq i<j \leq N} \vartheta\left(x_{i}-x_{j}\right) .
$$

For the case $N=2$, corresponding to the $\mathrm{SO}(5)$ theory, this function is illustrated in figure 6 .

To find the minima of the above function, we need the following result. For $-1 / 2 \leq$ $x_{i} \leq 1 / 2$,

$$
\begin{gathered}
(2 N-2) \sum_{1 \leq j \leq N} \vartheta\left(x_{j}\right)-\sum_{1 \leq i<j \leq N} \vartheta\left(x_{i}+x_{j}\right)-\sum_{1 \leq i<j \leq N} \vartheta\left(x_{i}-x_{j}\right)=2 \sum_{1 \leq i<j \leq N} \min \left(\left|x_{i}\right|,\left|x_{j}\right|\right) \\
=2(N-1) \min \left(\left|x_{i}\right|\right)+2(N-2) \min _{2}\left(\left|x_{i}\right|\right)+\cdots+2 \min _{N-1}\left(\left|x_{i}\right|\right)
\end{gathered}
$$

where $\min \left(\left|x_{i}\right|\right)$ stands for the smallest of $\left|x_{1}\right|, \ldots,\left|x_{N}\right|$, while $\min _{2}\left(\left|x_{i}\right|\right)$ stands for the next to smallest element, and so on. To prove (3.51), one can first verify it for $N=2$, and then use induction for $N>2$. 
Applying (3.51) we find that the Rains function in (3.50) is minimized to zero when one (and only one) of the $x_{j}$ is nonzero, and the rest are zero. This follows from the fact that $\max \left(\left|x_{i}\right|\right)$ does not show up on the r.h.s. of (3.51). Therefore, unlike for the theories of the previous subsection, here the matrix-integral is not localized around the origin of the $x_{i}$ space, but localized around the axes. Equation (3.47) thus simplifies to

$$
\ln Z_{\mathrm{SO}(2 N+1)}^{\mathrm{SUSY}}(b, \beta)=-\mathcal{E}_{0}^{D K}(b, \beta)+\ln \left(\frac{2 \pi}{\beta}\right)+O(1) \quad(\text { as } \beta \rightarrow 0) .
$$

More precise asymptotics. Below we improve the asymptotic relation (3.52) by obtaining the $O(1)$ term in it. The following discussion is somewhat detailed, and the reader not interested in the technical nuances is invited to skip to eq. (3.59) and continue reading from there.

Symmetry implies that we can compute the contribution from around the $x_{1}$ axis, and multiply the result by $N$ to get the final result. Since away from the axes the integrand is exponentially small, to compute the contribution coming from around the $x_{1}$ axis we can assume $\left|x_{2}\right|, \ldots,\left|x_{N}\right|<\epsilon$; this is the tails pruning. Unlike in the previous subsection though, now there is one direction (namely $x_{1}$ ) in which we can not prune.

Neglect for the moment the region where $\left|x_{1}\right|$ is smaller than or equal to some small fixed $\varepsilon_{1}>0$. Then for all the gamma functions that contain $x_{1}$ in their argument we can use the following estimate, valid uniformly over (fixed, $\beta$-independent) compact subsets of $\mathbb{R}$ avoiding $\mathbb{Z}$ (cf. Proposition 2.11 in [13]):

$$
\ln \Gamma\left(x+r\left(\frac{\sigma+\tau}{2}\right) ; \sigma, \tau\right) \sim 2 \pi i Q_{+}\left(\{x\}+r\left(\frac{\sigma+\tau}{2}\right) ; \sigma, \tau\right), \quad(\text { as } \beta \rightarrow 0, \text { for } x \in \mathbb{R} \backslash \mathbb{Z})
$$

where $r$ can be any (fixed) real number, and

$$
\begin{aligned}
Q_{+}(x ; \sigma, \tau)= & -\frac{x^{3}}{6 \tau \sigma}+\frac{\tau+\sigma+1}{4 \tau \sigma} x^{2}-\frac{\tau^{2}+\sigma^{2}+3 \tau \sigma+3 \tau+3 \sigma+1}{12 \tau \sigma} x \\
& +\frac{1}{24}(\tau+\sigma+1)\left(1+\tau^{-1}+\sigma^{-1}\right) .
\end{aligned}
$$

Using the estimate (2.16) for all the rest of the elliptic gamma functions (namely, those that do not contain $x_{1}$ in their argument), we obtain various factors of $e^{2 \pi i R_{0}}$, as well as one hyperbolic gamma for each of the elliptic gammas. It turns out that after using the aforementioned estimates the dependence of the integrand on $x_{1}$ completely drops out (this is essentially because (3.51) is independent of $\left.\max \left(\left|x_{i}\right|\right)\right)$. Therefore the integral over $x_{1}$ can be performed. The result is ( $R_{h}^{\text {integrand }}$ stands for the sum of the various $Q_{+}$es and $\left.R_{0} \mathrm{~s}\right)$

$$
\begin{aligned}
Z_{\mathrm{SO}(2 N+1)}^{\mathrm{SUSY}}(b, \beta) \approx & e^{-\beta E_{\text {susy }}(b) \frac{(p ; p)^{N}(q ; q)^{N}}{2^{N}(N-1) !} \Gamma^{N_{f}}\left((p q)^{r / 2}\right) \times e^{2 \pi i R_{h}^{\text {integrand }}} \times \int \mathrm{d}^{N-1} x} \\
& \frac{\prod_{j=2}^{N} \Gamma_{h}^{N_{f}}\left(\omega r \pm \frac{2 \pi x_{j}}{\beta}\right)}{\prod_{j=2}^{N} \Gamma_{h}\left( \pm \frac{2 \pi x_{j}}{\beta}\right) \prod_{2 \leq i<j \leq N}\left(\Gamma_{h}\left( \pm \frac{2 \pi\left(x_{i}+x_{j}\right)}{\beta}\right) \Gamma_{h}\left( \pm \frac{2 \pi\left(x_{i}-x_{j}\right)}{\beta}\right)\right)},
\end{aligned}
$$


with all the $N-1$ integrals over size $\epsilon$ neighborhoods of the axes $x_{i \neq 1}=0$. Note that the denominator of the prefactor is now $(N-1)$ !, because we multiplied by $N$ to take into account the contribution to the matrix-integral coming from all the other axes $x_{i \neq 1}$.

In the matrix-integral of (3.55), there remains temperature-dependence through hyperbolic gamma functions whose argument contains $x_{j \neq 1} / \beta$. To remove this dependence, we re-scale the $N-1$ variables $x_{j \neq 1}$, by using $N-1$ out of the $N$ factors of $\left(\frac{\beta}{2 \pi}\right)$ that the asymptotics of the Pochhammer symbols in the prefactor provide. After the re-scaling, the resulting integrals range over size $\epsilon / \beta$ neighborhoods of $x_{i \neq 1}=0$. Thus, although there is no temperature-dependence in the integrand, now the ranges of the integrals depend on $\beta$. To remove this latter dependence we need to argue that at large $x_{i \neq 1}(\propto 1 / \beta)$ the integrand of (3.55) is exponentially small (of the type $e^{-1 / \beta}$ ), and therefore tails completion introduces negligible error. Here the asymptotics of the hyperbolic gamma (2.19) can be used to show that for large $x_{i \neq 1}$ the asymptotics of the integrand of (3.55) is

$$
\begin{gathered}
\frac{\prod_{j=2}^{N} \Gamma_{h}^{N_{f}}\left(\omega r \pm \frac{2 \pi x_{j}}{\beta}\right)}{\prod_{j=2}^{N} \Gamma_{h}\left( \pm \frac{2 \pi x_{j}}{\beta}\right) \prod_{2 \leq i<j \leq N}\left(\Gamma_{h}\left( \pm \frac{2 \pi\left(x_{i}+x_{j}\right)}{\beta}\right) \Gamma_{h}\left( \pm \frac{2 \pi\left(x_{i}-x_{j}\right)}{\beta}\right)\right)} \\
\approx \exp \left(-2 \pi\left(\frac{b+b^{-1}}{2}\right) \tilde{u}(\mathbf{x})\right),
\end{gathered}
$$

with

$$
\tilde{u}(\mathbf{x}):=(2 N-2) \sum_{j=2}^{N}\left|x_{j}\right|-\sum_{2 \leq i<j \leq N}\left|x_{i}+x_{j}\right|-\sum_{2 \leq i<j \leq N}\left|x_{i}-x_{j}\right|
$$

We now need the following corollary of (3.51):

$$
\begin{gathered}
2 N \sum_{1 \leq j \leq N}\left|x_{j}\right|-\sum_{1 \leq i<j \leq N}\left|x_{i}+x_{j}\right|-\sum_{1 \leq i<j \leq N}\left|x_{i}-x_{j}\right|=2 \sum_{1 \leq i<j \leq N} \min \left(\left|x_{i}\right|,\left|x_{j}\right|\right)+2 \sum_{1 \leq j \leq N}\left|x_{j}\right| \\
=2 N \min \left(\left|x_{i}\right|\right)+2(N-1) \min _{2}\left(\left|x_{i}\right|\right)+\cdots+4 \min _{N-1}\left(\left|x_{i}\right|\right)+2 \max \left(\left|x_{i}\right|\right) .
\end{gathered}
$$

The relation (3.58) guarantees that $\tilde{u}(\mathbf{x})$ is strictly positive for nonzero $\mathbf{x}$, and that it is proportional to $\Lambda$ when $x_{i}= \pm \Lambda$. The tails completion of the matrix-integral in (3.55) is thus (exponentially) safe.

The asymptotic analysis is straightforward from here: since $x_{1}$ did not need re-scaling, one out of the $N$ factors of $\left(\frac{\beta}{2 \pi}\right)$ coming from the prefactor remains. This one factor of $\left(\frac{\beta}{2 \pi}\right)$, along with the exponential pieces of the asymptotics of the Pochhammer symbols in (3.49), and all the $e^{2 \pi i R_{0}}$ factors coming from the elliptic gamma functions in (3.49), provide the leading asymptotics of the $\mathrm{SO}(N)$ SQCD partition function.

One of the assumptions we used to arrive at the above conclusion was that the contribution of the matrix-integral from the region $\left|x_{1}\right|<\varepsilon_{1}$ was negligible. To justify this assumption we argue as follows. Since $\varepsilon_{1}$ can be taken to be arbitrarily small, and since the error we have introduced by using (3.53) in the integrand of (3.49) is uniformly bounded as $\beta \rightarrow 0$, the contribution to the matrix-integral from the region of size $\varepsilon_{1}$ is an $o(1)$ factor of the contribution we have computed so far. Thus our assumption, that the "small region" can be neglected when computing the asymptotics of the partition function, is valid with relative error which is $o(1)$. 
All in all, we find the following asymptotic relation:

$$
\ln Z_{S O(2 N+1)}^{\mathrm{SUSY}}(b, \beta)=-\mathcal{E}_{0}^{D K}(b, \beta)+\ln \left(\frac{2 \pi}{\beta}\right)+\ln Y_{3 d}(b)+o(1), \quad(\text { as } \beta \rightarrow 0)
$$

where

$$
Y_{3 d}(b)=\frac{\Gamma_{h}^{N_{f}}(\omega r)}{2^{N}(N-1) !} \int \mathrm{d}^{N-1} x \frac{\prod_{j=1}^{N-1} \Gamma_{h}^{N_{f}}\left(\omega r \pm x_{j}\right)}{\prod_{j=1}^{N-1} \Gamma_{h}\left( \pm x_{j}\right) \prod_{1 \leq i<j \leq N-1}\left(\Gamma_{h}\left( \pm\left(x_{i}+x_{j}\right)\right) \Gamma_{h}\left( \pm\left(x_{i}-x_{j}\right)\right)\right)},
$$

with the $x_{j}$ integrals going over the whole real line.

Take now the special case of the $\mathrm{SO}(3)$ theory with two flavors; i.e. $N=1, N_{f}=2$. Set moreover $b=1$. The asymptotic expression (3.59) simplifies in this case to

$$
\ln Z_{S O(3)}^{\mathrm{SUSY}}(\beta)=\ln \left(\frac{2 \pi}{\beta}\right)+\ln \left(\frac{\Gamma_{h}^{2}(i r ; i, i)}{2}\right)+o(1), \quad(\text { as } \beta \rightarrow 0)
$$

with $r=1 / 2$. Note that there is no $1 / \beta$ term on the r.h.s., because the $\mathrm{SO}(3)$ theory has $\operatorname{Tr} R=0$ (and also $L_{h \text { min }}=0$ ). Employing

$$
\ln \Gamma_{h}(i x ; i, i)=(x-1) \ln \left(1-e^{-2 \pi i x}\right)-\frac{1}{2 \pi i} L i_{2}\left(e^{-2 \pi i x}\right)+\frac{i \pi}{2}(x-1)^{2}-\frac{i \pi}{12},
$$

and noting $L i_{2}(-1)=-\pi^{2} / 12$, we find that $\Gamma_{h}(i / 2 ; i, i)=1 / \sqrt{2}$. Therefore (3.61) can be further simplified to

$$
\ln Z_{S O(3)}^{\mathrm{SUSY}}(\beta)=\ln \left(\frac{2 \pi}{\beta}\right)-2 \ln 2+o(1) \quad(\text { as } \beta \rightarrow 0) .
$$

Much more precise asymptotics for the $\mathrm{SO}(3)$ theory with $N_{f}=2$ when $b=1$. Luckily, the asymptotic expansion in (3.63) can be completed to all orders, with the result

$$
\ln Z_{S O(3)}^{\mathrm{SUSY}}(\beta) \sim \ln \left(\frac{\pi}{2 \beta}-\frac{1}{2 \pi}\right) \quad(\text { as } \beta \rightarrow 0) .
$$

To derive the above all-orders asymptotics, we first note the following remarkable coincidence: the SUSY partition function of the $\mathrm{SO}(3)$ theory with two flavors precisely matches the $v=(p q)^{1 / 6}$ specialization of the $\mathcal{N}=2$ partition function of the $\mathrm{SU}(2) \mathcal{N}=4$ theory, to be described in the next section. In particular, when $b=1$, the said $\mathcal{N}=2$ partition function becomes the Schur partition function of the $\mathrm{SU}(2) \mathcal{N}=4$ theory, and the latter is exceptionally well under control. The result in (3.64) is what one gets for $\ln Z_{\mathrm{SU}(2)}^{\mathrm{Schur}} \mathcal{N}=4$, as demonstrated in appendix B.

\subsection{2 $\mathrm{SU}(N) \mathcal{N}=4 \mathrm{SYM}$}

The $\mathcal{N}=4$ theory is another important example with high-temperature Coulomb branch decompactification on $S^{3} \times S^{1}$. 
The $\mathrm{SU}(N)$ theory has the following SUSY partition function [51]:

$$
\begin{aligned}
Z_{\mathcal{N}=4}^{\operatorname{SUSY}}(b, \beta)=e^{-\beta E_{\text {susy }}(b)} & \frac{(p ; p)^{N-1}(q ; q)^{N-1}}{N !} \Gamma^{3(N-1)}\left((p q)^{1 / 3}\right) \\
& \times \int \mathrm{d}^{N-1} x \prod_{1 \leq i<j \leq N} \frac{\Gamma^{3}\left((p q)^{1 / 3}\left(z_{i} / z_{j}\right)^{ \pm 1}\right)}{\Gamma\left(\left(z_{i} / z_{j}\right)^{ \pm 1}\right)},
\end{aligned}
$$

with $\prod_{i=1}^{N} z_{i}=1$.

Recall that for the theories of the previous subsection, the integrand of the matrixintegral was everywhere exponentially smaller than in the origin of the $x_{i}$ space; in other words, the integral localized at a point. In the $\mathrm{SO}(N)$ SQCD case, we saw that the integral localizes around the (one-real-dimensional) axes of the $x_{i}$ space. We will shortly find that for the $\mathcal{N}=4$ theory the matrix-integral does not localize at all.

The Rains function of the theory is

$$
L_{h}^{\mathcal{N}=4}=3\left(1-\frac{2}{3}\right) \sum_{1 \leq i<j \leq N} \vartheta\left(x_{i}-x_{j}\right)-\sum_{1 \leq i<j \leq N} \vartheta\left(x_{i}-x_{j}\right)=0 .
$$

In other words, there is no effective potential for the interaction of the holonomies, and the matrix-integral does not localize: $\mathfrak{h}_{q u}=\mathfrak{h}_{c l}$. Eq. (3.47) thus dictates

$$
\ln Z_{\mathcal{N}=4}^{\mathrm{SUSY}}(b, \beta)=(N-1) \ln \left(\frac{2 \pi}{\beta}\right)+O\left(\beta^{0}\right) .
$$

There is no $O(1 / \beta)$ term on the r.h.s., because $\operatorname{Tr} R=0$ for the $\mathcal{N}=4$ theory (and also $\left.L_{h \min }=0\right)$.

More precise asymptotics. Neglecting the contribution to the integral coming from a small (size $\varepsilon_{1}$ ) neighborhood of $\mathcal{S}_{g}$ (see the similar discussion for the $\mathrm{SO}(N)$ SQCD theory above), we can use the estimate (3.53) for all the gamma functions in the integrand of (3.65), and obtain that the integrand is in fact approximately equal to one. Therefore the integral is asymptotically equal to $\operatorname{vol}\left(\mathfrak{h}_{c l}\right)=1$, and the asymptotic analysis of $Z_{\mathcal{N}=4}^{\text {SUSY }}$ becomes trivial: the only contributions that are $O(1)$ or larger come from the integral's prefactor. These can be estimated using (2.9) and (2.16). All in all, we find

$$
\ln Z_{\mathcal{N}=4}^{\mathrm{SUSY}}(b, \beta)=(N-1) \ln \left(\frac{2 \pi}{\beta}\right)+3(N-1) \ln \Gamma_{h}\left(\frac{2}{3} \omega\right)-\ln N !+o(1) \quad(\text { as } \beta \rightarrow 0) .
$$

\subsubsection{The $\mathbb{Z}_{2}$ orbifold theory}

We now study a quiver gauge theory, to illustrate how easily Rains's method generalizes to theories with more than one simple factor in their gauge group.

Consider the $\mathbb{Z}_{2}$ orbifold of the $\mathcal{N}=4 \mathrm{SYM}$ with $\mathrm{SU}(N)$ gauge group. The theory consists of two $\mathrm{SU}(N)$ gauge groups, with one chiral multiplet in the adjoint of each, and one doublet of bifundamental chiral multiplets from each gauge group to the other. All the chiral multiplets have R-charge $r=2 / 3$. 


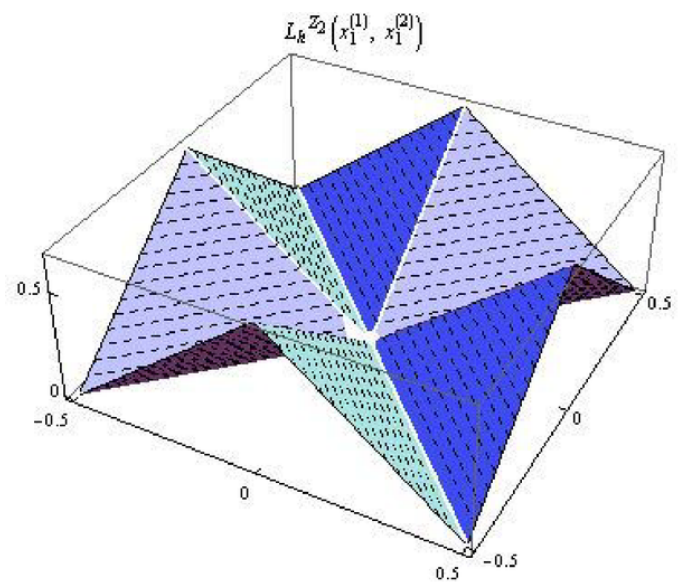

Figure 7. The Rains function of the $\mathrm{SU}(2) \times \mathrm{SU}(2)$ orbifold theory. Note the flat directions along $\left|x_{1}^{(1)}\right|=\left|x_{1}^{(2)}\right|$.

The SUSY partition function is given by (cf. [52])

$$
\begin{array}{r}
Z_{\mathbb{Z}_{2}}^{\mathrm{SUSY}}(b, \beta)=e^{-\beta E_{\text {susy }}(b)}\left(\prod _ { k = 1 , 2 } \left[\frac{(p ; p)^{N-1}(q ; q)^{N-1}}{N !} \Gamma^{N-1}\left((p q)^{1 / 3}\right) \int \mathrm{d}^{N-1} x^{(k)}\right.\right. \\
\left.\left.\left(\prod_{1 \leq i<j \leq N} \frac{\Gamma\left((p q)^{1 / 3}\left(z_{i}^{(k)} / z_{j}^{(k)}\right)^{ \pm 1}\right)}{\Gamma\left(\left(z_{i}^{(k)} / z_{j}^{(k)}\right)^{ \pm 1}\right)}\right)\right]\right) \times \prod_{i, j=1}^{N}\left(\Gamma\left((p q)^{1 / 3}\left(z_{i}^{(1)} / z_{j}^{(2)}\right)^{ \pm 1}\right)\right),
\end{array}
$$

with $\prod_{i=1}^{N} z_{i}^{(1)}=\prod_{i=1}^{N} z_{i}^{(2)}=1$.

The Rains function of the theory is

$$
L_{h}^{\mathbb{Z}_{2}}\left(\mathbf{x}^{(1)}, \mathbf{x}^{(2)}\right)=-\frac{2}{3} \sum_{1 \leq i<j \leq N} \vartheta\left(x_{i}^{(1)}-x_{j}^{(1)}\right)-\frac{2}{3} \sum_{1 \leq i<j \leq N} \vartheta\left(x_{i}^{(2)}-x_{j}^{(2)}\right)+\frac{2}{3} \sum_{i, j=1}^{N} \vartheta\left(x_{i}^{(1)}-x_{j}^{(2)}\right) .
$$

For the case $N=2$, corresponding to the $\mathrm{SU}(2) \times \mathrm{SU}(2)$ theory, this function is illustrated in figure 7.

The generalized triangle inequality (2.20) applies with $c=x^{(1)}, d=x^{(2)}$, and implies that $L_{h}^{\mathbb{Z}_{2}}$ is positive semi-definite. It moreover shows that $L_{h}^{\mathbb{Z}_{2}}$ vanishes if the $x_{i}^{(1)}, x_{j}^{(2)}$ can be permuted such that either of (2.21) or (2.22) holds. For simplicity we consider all $x_{i}^{(1)}$ to be positive and very small, except for $x_{N}^{(1)}=-x_{1}^{(1)}-\cdots-x_{N-1}^{(1)}$ being negative and very small, and similarly for $x_{j}^{(2)}$. Assuming either (2.21) or (2.22), we conclude that $x_{i}^{(1)}=x_{i}^{(2)}$. Based on this result, and also the $N=2$ case whose Rains function is displayed in figure 7 , we conjecture that for the $\mathbb{Z}_{2}$ orbifold theory $\operatorname{dimh} \mathfrak{h}_{q u}=N-1$, and thereby

$$
\ln Z_{\mathbb{Z}_{2}}^{\mathrm{SUSY}}(b, \beta)=-\mathcal{E}_{0}^{D K}(b, \beta)+(N-1) \ln \left(\frac{2 \pi}{\beta}\right)+O(1) \quad(\text { as } \beta \rightarrow 0) .
$$

\subsubsection{The $\mathbb{Z}_{3}$ orbifold theory}

The $\mathrm{SU}(N)^{3}$ quiver is our second (and last) example with $Q_{h} \neq 0$. More precisely, it is for $N>2$ that the model is chiral, and has nonzero $Q_{h}$, since the fundamental and anti- 
fundamental representations of $\mathrm{SU}(2)$ are equivalent. The quiver has three chiral multiplets with R-charge $2 / 3$ going from the first node to the second, a similar triplet going from the second node to the third, and a last triplet going from to the third node to the first.

Similarly to the case of the $\mathbb{Z}_{2}$ orbifold theory $i$ ) Rains's generalized triangle inequality (2.20) establishes that $L_{h}^{\mathbb{Z}_{3}}$ is positive semi-definite, and $i i$ ) based on an argument made in the region where $x_{i}^{(1,2,3)}$ are small (and positive except for $i=N$ ) we conjecture that also for this theory $\operatorname{dim} \mathfrak{h}_{q u}=N-1$.

Although $Q_{h}^{\mathbb{Z}_{3}}$ does not identically vanish for $N>2$, our numerical investigation for $N=3$ indicates that it vanishes on $\mathfrak{h}_{q u}$, and we suspect $Q_{h}^{\mathbb{Z}_{3}}$ to keep vanishing on $\mathfrak{h}_{q u}$ for all $N \geq 3$. This suggests that the nonzero $Q_{h}$ function of the $\mathbb{Z}_{3}$ orbifold theory does not affect the leading high-temperature asymptotics of its SUSY partition function. We are thus led to conjecture

$$
\ln Z_{\mathbb{Z}_{3}}^{\mathrm{SUSY}}(b, \beta)=-\mathcal{E}_{0}^{D K}(b, \beta)+(N-1) \ln \left(\frac{2 \pi}{\beta}\right)+O(1) \quad(\text { as } \beta \rightarrow 0) .
$$

\section{3 $Z_{S^{3}}$ exponentially divergent (or: the curious case of the SCFTs with $c<a)$}

In this subsection we consider examples of Lagrangian SCFTs arising as IR fix points of R-symmetric SUSY gauge theories with a semi-simple gauge group, with $Q_{h}=0$, and with $\operatorname{Tr} R>0$.

We write the asymptotics in terms of the central charges $a$ and $c$ of our theories. Since $\operatorname{Tr} R=-16(c-a)$, the Di Pietro-Komargodski formula for SCFTs reads

$$
\ln Z^{\mathrm{SUSY}}(b, \beta) \approx \frac{16 \pi^{2}}{3 \beta}\left(\frac{b+b^{-1}}{2}\right)(c-a) \quad(\text { as } \beta \rightarrow 0) .
$$

On the other hand, the formula (3.30) becomes

$$
\ln Z^{\mathrm{SUSY}}(b, \beta)=\frac{16 \pi^{2}}{3 \beta}\left(\frac{b+b^{-1}}{2}\right)\left(c-a-\frac{3}{4} L_{h} \min \right)+\operatorname{dimh}_{q u} \ln \left(\frac{2 \pi}{\beta}\right)+O\left(\beta^{0}\right),
$$

with $L_{h}$ min the minimum of the Rains function over $\mathfrak{h}_{c l}$. Note that the leading piece takes the same form as the Di Pietro-Komargodski formula, but with the "shifted $c-a$ " defined as $(c-a)_{\text {shifted }}:=c-a-\frac{3}{4} L_{h \text { min }}$; this last relation appears to be analogous to the equation $c_{\text {eff }}=c-24 h_{\min }$ frequently discussed in the context of non-unitary $2 \mathrm{~d}$ CFTs (see e.g. [53]).

For SCFTs with $c<a$, the r.h.s. of the Di Pietro-Komargodski formula (3.73) becomes negative. Interestingly, in the SCFTs with $c<a$ studied below, the correction term $-\frac{3}{4} L_{h}$ min makes the r.h.s. of (3.74) positive. In other words $(c-a)_{\text {shifted }}>0$.

In the theories studied in this subsection, $\mathbf{x}=0$ is not a local minimum of $L_{h}$; it is in fact a local maximum. We now argue that when $\mathbf{x}=0$ is not a local minimum of the Rains function, $Z_{S^{3}}(b ; \Lambda)$ defined in (3.37) diverges exponentially in $\Lambda$ as $\Lambda \rightarrow \infty$.

Our starting point for the argument is the relation (we are assuming $Q_{h}=0$ )

$$
Z_{S^{3}}(b ; \Lambda) \approx \frac{1}{|W|} \int_{\Lambda} \mathrm{d}^{r} x e^{-2 \pi\left(\frac{b+b^{-1}}{2}\right) \tilde{L}_{S^{3}}(\mathbf{x})},
$$




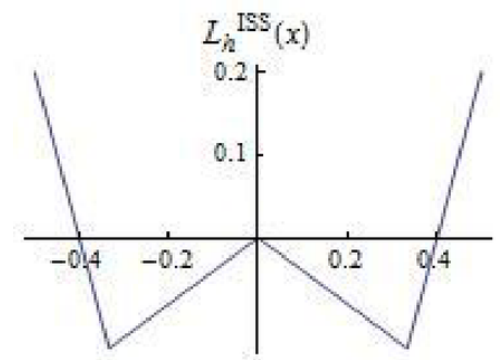

Figure 8. The Rains function of the SU(2) ISS theory. Note that the minima lie at $x= \pm 1 / 3$.

which we obtained in subsection 3.2. The assumption that $\mathbf{x}=0$ is not a local minimum implies that there are neighboring points of $\mathbf{x}=0$ where the effective potential, and hence the Rains function, is negative. Since for small enough $\mathbf{x}$ the Rains function and $\tilde{L}_{S^{3}}$ coincide, we learn that there are $\mathbf{x} \neq 0$ points where $\tilde{L}_{S^{3}}$ is negative. Since $\tilde{L}_{S^{3}}$ is a homogenous function of $\mathbf{x}$, we conclude that there are directions along which we can take $|\mathbf{x}| \propto \Lambda$ and have $\tilde{L}_{S^{3}}(\mathbf{x}) \propto-\Lambda$. The integrand of (3.75) would become exponentially large if $|\mathbf{x}|$ becomes large along those directions, and $Z_{S^{3}}(b ; \Lambda)$ would diverge exponentially in $\Lambda$.

\subsubsection{The SU(2) ISS model}

There are two famous interacting Lagrangian SCFTs with $c<a$. The first is the Intriligator-Seiberg-Shenker (ISS) model of dynamical SUSY breaking [16]. The theory is formulated in the $\mathrm{UV}$ as an $\mathrm{SU}(2)$ vector multiplet with a single chiral multiplet in the four-dimensional representation of the gauge group. Although originally suspected to confine (and to break supersymmetry upon addition of a tree-level superpotential) [16], the theory is currently believed to flow to an interacting SCFT in the IR [54,55], where the chiral multiplet has R-charge $3 / 5$. The IR SCFT has $c-a=-7 / 80$.

The SUSY partition function of this theory is (cf. [56])

$$
Z_{\mathrm{ISS}}^{\mathrm{SUSY}}(b, \beta)=e^{-\beta E_{\text {susy }}(b)} \frac{(p ; p)(q ; q)}{2} \int \mathrm{d} x \frac{\Gamma\left((p q)^{3 / 10} z^{ \pm 1}\right) \Gamma\left((p q)^{3 / 10} z^{ \pm 3}\right)}{\Gamma\left(z^{ \pm 2}\right)} .
$$

The Rains function of the theory is

$$
L_{h}^{\mathrm{ISS}}(x)=\frac{2}{5} \vartheta(x)+\frac{2}{5} \vartheta(3 x)-\vartheta(2 x) .
$$

This function is plotted in figure 8 .

A direct examination reveals that $L_{h}^{\mathrm{ISS}}(x)$ is minimized at $x= \pm 1 / 3$, and $L_{h}^{\mathrm{ISS}}( \pm 1 / 3)=$ $-2 / 15$. The asymptotics of $Z_{\text {ISS }}^{\text {SUSY }}$ is hence given according to (3.74) by

$$
\ln Z_{\mathrm{ISS}}^{\mathrm{SUSY}}(b, \beta)=\frac{\pi^{2}}{15 \beta}\left(\frac{b+b^{-1}}{2}\right)+O\left(\beta^{0}\right) .
$$

In other words we have $(c-a)_{\text {shifted }}=c-a+1 / 10=1 / 80$. 
Much more precise asymptotics. We now proceed to improve the asymptotic relation (3.78) to all-orders accuracy. The reader not interested in the technical details is invited to skip to the relation (3.82) and continue reading from there.

We know that the integral (3.76) localizes around $x= \pm 1 / 3$ at high temperatures. Therefore we prune down the integration range to two small neighborhoods of size $\epsilon$ around $x=1 / 3$ and $x=-1 / 3$. The $x \rightarrow-x$ symmetry then implies that we can compute only the integral around $x=1 / 3$, and multiply the result by two.

In an $O(\epsilon)$ neighborhood around $x=1 / 3$, the arguments of the gamma functions $\Gamma\left((p q)^{3 / 10} z^{ \pm 1}\right)$ and $\Gamma\left(z^{ \pm 2}\right)$ inside the integrand of (3.76) are such that the estimate (3.53) applies to them. But the gamma functions $\Gamma\left((p q)^{3 / 10} z^{ \pm 3}\right)$ need special care now: for $x \geq 1 / 3$ their argument is such that we can not use the central estimate (2.16) for them. To get around this, as mentioned below (2.17) we can replace every $x$ on the r.h.s. of (2.16) with $\{x\}$, to obtain (after scaling $x \mapsto 3 x$ )

$$
\begin{array}{r}
\Gamma\left((p q)^{r / 2} z^{ \pm 3}\right) \simeq e^{2 \pi i R_{0}\left(\frac{\beta}{2 \pi} \omega r \pm 3 x ; \sigma, \tau\right)} \Gamma_{h}\left(r \omega \pm \frac{2 \pi(3 x-1)}{\beta}\right) \\
e^{2 \pi i(1-r) \frac{2 \pi \omega}{\beta}(6 x-1)} . \\
\quad(\text { For } 0<3 x<2 .)
\end{array}
$$

The extended range of applicability of the above estimate allows us to approximate $\Gamma\left((p q)^{3 / 10} z^{ \pm 3}\right)$ for $x \geq 1 / 3$, and also uniformly on the $O(\epsilon)$ neighborhood of $x=1 / 3$, which is where (half of) the dominant contribution to the integral (3.76) comes from (the other half comes from an $O(\epsilon)$ neighborhood of $x=-1 / 3)$.

Using the estimates (2.9), (3.79), and (3.53), defining a new variable $x^{\prime}:=x-1 / 3$, and then re-scaling $x^{\prime} \mapsto x^{\prime} /(\beta / 2 \pi)$, we find that $Z_{\mathrm{ISS}}^{\mathrm{SUSY}}(b, \beta)$ in (3.76) simplifies to

$$
Z_{\mathrm{ISS}}^{\mathrm{SUSY}}(b, \beta) \simeq e^{\frac{16 \pi^{2}}{3 \beta}\left(c-a+\frac{1}{10}\right)\left(\frac{b+b^{-1}}{2}\right)} \times Y_{S^{3}}^{\mathrm{ISS}}(b ; 2 \pi \epsilon / \beta),
$$

with

$$
Y_{S^{3}}^{\mathrm{ISS}}(b ; 2 \pi \epsilon / \beta)=\int_{-2 \pi \epsilon / \beta}^{2 \pi \epsilon / \beta} \mathrm{d} x^{\prime} e^{-\frac{4 \pi}{5}\left(b+b^{-1}\right) x^{\prime}} \times \Gamma_{h}\left(3 x^{\prime}+(3 / 5) \omega\right) \Gamma_{h}\left(-3 x^{\prime}+(3 / 5) \omega\right) .
$$

The asymptotics of the hyperbolic gamma in (2.19) guarantees that the integrand in the above equation is exponentially small at large $|x|$, and hence we can safely complete the tails to the whole real line.

Our final asymptotic estimate is obtained by taking the logarithm of (3.80):

$$
\ln Z_{\mathrm{ISS}}^{\mathrm{SUSY}}(b, \beta) \sim \frac{16 \pi^{2}}{3 \beta}(c-a)_{\text {shifted }}\left(\frac{b+b^{-1}}{2}\right)+\ln Y_{S^{3}}^{\mathrm{ISS}}(b), \quad(\text { as } \beta \rightarrow 0)
$$

with $Y_{S^{3}}^{\mathrm{ISS}}(b)=Y_{S^{3}}^{\mathrm{ISS}}(b ; \infty)$, and $(c-a)_{\text {shifted }}=(c-a)+1 / 10=1 / 80$.

Now, if $Y_{S^{3}}^{\text {ISS }}(b)$ were found to vanish, then the $O\left(\beta^{0}\right)$ term on the r.h.s. of (3.82) would diverge, the relation (3.82) would not make sense, and we would need to redo the asymptotic analysis of $Z_{\mathrm{ISS}}^{\mathrm{SUS}}(b, \beta)$ more carefully; the careful analysis would then presumably lead us to an asymptotics different from the one given by (3.30); that would be a scenario 
exemplifying the subtle cancelations discussed below (3.15) [in the present case, the cancelation would be seen at the level of $\left.Y_{S^{3}}^{\text {ISS }}(b)\right]$, and their consequential failure of (3.30). However, it follows form (2.4) that the product of the hyperbolic gammas inside the integrand in (3.81) is (real and) positive, and thus $Y_{S^{3}}^{\text {ISS }}(b)>0$. Therefore the unexpected cancelations discussed below (3.15) do not occur here.

An analysis similar to the one above can be performed for any SUSY gauge theory with a semi-simple gauge group and non-chiral matter content ${ }^{6}$ (hence $Q_{h}=0$ ), whose Rains function is minimized on a set of points not consisting only of the origin (i.e. $\operatorname{dim} \mathfrak{h}_{q u}=0$ and $\left.\mathfrak{h}_{q u} \backslash\{\mathbf{x}=0\} \neq \varnothing\right)$. All such theories would display asymptotics similar to (3.82). In particular, $\operatorname{dimh} \mathfrak{h}_{q u}=0$ implies that the high-temperature expansion of $\ln Z^{\mathrm{SUSY}}(b, \beta)$ (and in fact also that of $\ln Z^{\mathrm{SUSY}}\left(b, \beta ; m_{a}\right)$ ) terminates at $O\left(\beta^{0}\right)$. We showed the latter statement in subsection 3.1 for theories whose Rains function is minimized only at the origin, irrespective of whether their $Q_{h}$ was zero or not. It would be interesting to prove (or disprove) the same general statement for theories with $\operatorname{dim} \mathfrak{h}_{q u}=0, \mathfrak{h}_{q u} \backslash\{\mathbf{x}=0\} \neq \varnothing$, and nonzero $Q_{h}$.

\subsubsection{The $\mathrm{SO}(2 N+1)$ BCI model with $1<N<5$}

The second famous example of interacting SCFTs with $c<a$ is provided by the "misleading" $\mathrm{SO}(n)$ theory of Brodie, Cho, and Intriligator [17]. This is an $\mathcal{N}=1 \mathrm{SO}(n)$ gauge theory with a single chiral multiplet in the two-index symmetric traceless tensor representation of the gauge group. The theory is asymptotically free if $n \geq 5$. For $5 \leq n<11$ the corresponding interacting IR SCFT has $c-a=-(n-1) / 16$ (for greater values of $n$ the R-symmetry of the IR SCFT is believed to mix with an emergent accidental symmetry, and thus more care is called for; cf. [57]).

For the $\mathrm{SO}(2 N+1)$ theory (with $1<N<5$ ) we have (cf. [56])

$$
\begin{aligned}
Z_{\mathrm{BCI}}^{\mathrm{SUSY}}(b, \beta)= & e^{-\beta E_{\mathrm{susy}}(b) \frac{(p ; p)^{N}(q ; q)^{N}}{2^{N} N !} \Gamma^{N}\left((p q)^{2 /(2 N+3)}\right) \int \mathrm{d}^{N} x} \\
& \prod_{i<j} \frac{\Gamma\left((p q)^{2 /(2 N+3)} z_{i}^{ \pm 1} z_{j}^{ \pm 1}\right)}{\Gamma\left(z_{i}^{ \pm 1} z_{j}^{ \pm 1}\right)} \prod_{j=1}^{N} \frac{\Gamma\left((p q)^{2 /(2 N+3)} z_{j}^{ \pm 1},(p q)^{2 /(2 N+3)} z_{j}^{ \pm 2}\right)}{\Gamma\left(z_{j}^{ \pm 1}\right)}
\end{aligned}
$$

The Rains function of the theory is

$$
L_{h}^{\mathrm{BCI}}(x)=\frac{4}{2 N+3}\left(\left(\frac{2 N-1}{4}\right) \sum_{j} \vartheta\left(2 x_{j}\right)-\sum_{j} \vartheta\left(x_{j}\right)-\sum_{i<j} \vartheta\left(x_{i}+x_{j}\right)-\sum_{i<j} \vartheta\left(x_{i}-x_{j}\right)\right) .
$$

For $N=2$, corresponding to the $\mathrm{SO}(5)$ theory, this function is plotted in figure 9 .

\footnotetext{
${ }^{6}$ Non-chirality guarantees that the subtle cancelations discussed below (3.15) do not occur (cf. [45]); note, for example, how below (3.82) we argued for the positivity of the integrand of $Y_{S^{3}}^{\text {ISS }}(b)$, and thus for $Y_{S^{3}}^{\mathrm{ISS}}(b) \neq 0$.
} 


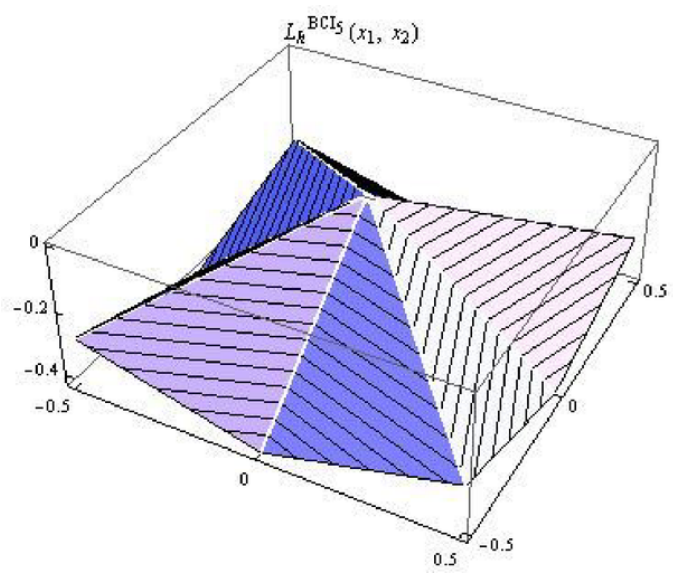

Figure 9. The Rains function of the $\mathrm{SO}(5) \mathrm{BCI}$ theory. Note that the function is maximized at the origin, and minimized at $\left(x_{1}, x_{2}\right)=(0, \pm 1 / 2)$ and $\left(x_{1}, x_{2}\right)=( \pm 1 / 2,0)$.

To find the minima of the above function, we need the following result, valid for $-1 / 2 \leq x_{i} \leq 1 / 2$ :

$$
\begin{aligned}
& \left(\frac{2 N-1}{4}\right) \sum_{1 \leq j \leq N} \vartheta\left(2 x_{j}\right)-\sum_{1 \leq j \leq N} \vartheta\left(x_{j}\right)-\sum_{1 \leq i<j \leq N} \vartheta\left(x_{i}+x_{j}\right)-\sum_{1 \leq i<j \leq N} \vartheta\left(x_{i}-x_{j}\right)= \\
& -\frac{3}{2} \sum_{i<j} \max \left(\left|x_{i}\right|,\left|x_{j}\right|\right)+\frac{1}{2} \sum_{i<j} \min \left(\left|x_{i}\right|,\left|x_{j}\right|\right)=\sum_{j}\left(-\frac{3 N}{2}+2 j-\frac{1}{2}\right) \min _{N-j+1}\left(\left|x_{i}\right|\right),
\end{aligned}
$$

with $\min _{N}\left(\left|x_{i}\right|\right):=\max \left(\left|x_{i}\right|\right)$. The proof of (3.85) is similar to that of (3.51).

Note that the coefficient of the $j$ th term on the r.h.s. of (3.85) is negative if $j<\frac{3 N+1}{4}$, and positive otherwise. This implies that the Rains function (3.84) is minimized when $\left\lfloor\frac{3 N+1}{4}\right\rfloor$ of the $\left|x_{i}\right|$ are maximized (i.e. $x_{i}= \pm 1 / 2$ ), and the rest of the $\left|x_{i}\right|$ are minimized (i.e. $x_{i}=0$ ). Consequently, the minimum of the Rains function is

$$
L_{h \min }^{\mathrm{BCI}}=-\frac{1}{2 N+3} \sum_{1 \leq j \leq\left\lfloor\frac{3 N+1}{4}\right\rfloor}(3 N+1-4 j) .
$$

This is less than zero for any $N>1$. Therefore the Di Pietro-Komargodski formula needs to be modified in the $\mathrm{SO}(2 N+1)$ BCI model with $1<N<5$.

For example, consider the $\mathrm{SO}(5)$ theory corresponding to $N=2$. This theory has $c-a=-1 / 4$. From eq. (3.86) we have in this case $L_{h}^{\mathrm{BCI}}$ min $(x)=-3 / 7$. The asymptotics of $Z^{\mathrm{SUSY}}$ is therefore given according to (3.74) by

$$
\ln Z_{B C I_{5}}^{\mathrm{SUSY}}(b, \beta)=\frac{8 \pi^{2}}{21 \beta}\left(\frac{b+b^{-1}}{2}\right)+O\left(\beta^{0}\right)
$$

In other words $(c-a)_{\text {shifted }}=c-a+9 / 28=1 / 14$. 
Much more precise asymptotics for the SO(5) BCI theory. We now proceed to improve (3.87) to all-orders accuracy. The reader not interested in technical details is invited to skip to the relation (3.91) and continue reading from there.

The matrix-integral of the $\mathrm{SO}(5) \mathrm{BCI}$ theory localizes around two points. We compute the contribution coming from around $\left(x_{1}, x_{2}\right)=( \pm 1 / 2,0)$, and multiply the result by two to take into account also the contribution coming from around $\left(x_{1}, x_{2}\right)=(0, \pm 1 / 2)$.

Analogously to (3.79), this time we need

$$
\begin{array}{r}
\Gamma\left((p q)^{r / 2} z_{1}^{ \pm 2}\right) \simeq e^{2 \pi i R_{0}\left(\frac{\beta}{2 \pi} \omega r \pm 2 x_{1} ; \sigma, \tau\right)} \Gamma_{h}\left(r \omega \pm \frac{2 \pi\left(2 x_{1}-1\right)}{\beta}\right) e^{2 \pi i(1-r) \frac{2 \pi \omega}{\beta}\left(4 x_{1}-1\right)} . \\
\quad\left(\text { For } 0<2 x_{1}<2 .\right)
\end{array}
$$

Proceeding as in the case of the ISS model, this time defining $x_{1}^{\prime}:=x_{1}-1 / 2$, pruning down to $\left|x_{1}^{\prime}\right|,\left|x_{2}\right|<\epsilon$ and then re-scaling $x_{1}^{\prime}, x_{2} \mapsto x_{1}^{\prime} /(\beta / 2 \pi), x_{2} /(\beta / 2 \pi)$, we arrive at

$$
Z_{B C I_{5}}^{\mathrm{SUSY}}(b, \beta) \simeq e^{\frac{16 \pi^{2}}{3 \beta}\left(c-a+\frac{9}{28}\right)\left(\frac{b+b^{-1}}{2}\right)} \times Y_{S^{3}}^{B C I_{5}}(b ; 2 \pi \epsilon / \beta), \quad(\text { as } \beta \rightarrow 0)
$$

with

$$
\begin{aligned}
Y_{S^{3}}^{B C I_{5}}(b ; \Lambda)= & \frac{1}{2} \int_{-\Lambda}^{\Lambda} \mathrm{d} x_{1}^{\prime} \Gamma_{h}\left((4 / 7) \omega \pm 2 x_{1}^{\prime}\right) \times \\
& \frac{\Gamma_{h}^{2}((4 / 7) \omega)}{2} \int_{-\Lambda}^{\Lambda} \mathrm{d} x_{2} \frac{\Gamma_{h}\left((4 / 7) \omega \pm x_{2}\right) \Gamma_{h}\left((4 / 7) \omega \pm 2 x_{2}\right)}{\Gamma_{h}\left( \pm x_{2}\right)} .
\end{aligned}
$$

The asymptotics of the hyperbolic gamma (2.19) guarantees that the tails completion is safe, and we obtain

$$
\ln Z_{B C I_{5}}^{\mathrm{SUSY}}(b, \beta) \sim \frac{16 \pi^{2}}{3 \beta}(c-a)_{\text {shifted }}\left(\frac{b+b^{-1}}{2}\right)+\ln Y_{S^{3}}^{B C I_{5}}(b), \quad(\text { as } \beta \rightarrow 0)
$$

with $Y_{S^{3}}^{B C I_{5}}(b)=Y_{S^{3}}^{B C I_{5}}(b ; \infty)$, and $(c-a)_{\text {shifted }}=(c-a)+9 / 28=1 / 14$. From $(2.4)$ it follows that the integrands in (3.90) are (real and) positive; therefore $Y_{S^{3}}^{B C I_{5}}(b)>0$, assuring that the unexpected cancelations discussed below (3.15) do not occur here either.

\section{Asymptotics of the $\mathcal{N}=2$ partition function}

In this section we focus on Lagrangian $\mathcal{N}=2$ SCFTs; these have the extended R-symmetry group $\mathrm{SU}(2)_{R_{\mathcal{N}=2}} \times \mathrm{U}(1)_{r_{\mathcal{N}=2}}$. The $\mathcal{N}=2$ theories are put on $S_{b}^{3} \times S_{\beta}^{1}$, and their pathintegral is computed in presence of a background gauge field that couples to a specific linear combination of $\mathrm{U}(1)_{r_{\mathcal{N}=2}}$ and the Cartan of $\mathrm{SU}(2)_{R_{\mathcal{N}=2}}$. Denoting the latter by $\mathrm{U}(1)_{R_{\mathcal{N}=2}}$, the said linear combination is

$$
Q_{v}=-\left(r_{\mathcal{N}=2}+R_{\mathcal{N}=2}\right) .
$$

The $\mathcal{N}=1$ R-symmetry is also a linear combination of $\mathrm{U}(1)_{R_{\mathcal{N}=2}}$ and $\mathrm{U}(1)_{r_{\mathcal{N}=2}}$; it is given by

$$
r=\frac{2}{3}\left(2 R_{\mathcal{N}=2}-r_{\mathcal{N}=2}\right)
$$


The linear combination in (4.1) can hence be written as

$$
Q_{v}=\frac{3}{2} r-3 R_{\mathcal{N}=2} .
$$

The path-integral computed in the presence of a background gauge field (along the $S_{\beta}^{1}$, with value $m_{v}$, and with holonomy $v=e^{i \beta m_{v}}$ ) coupling the linear combination (4.3) defines the $\mathcal{N}=2$ partition function $Z^{\mathcal{N}=2}\left(b, \beta, m_{v}\right)$. When the gauge group $G$ is semi-simple, and when besides the $\mathcal{N}=2$ vector multiplet the theory has chiral multiplets in the doublet of $\mathrm{SU}(2)_{R_{\mathcal{N}=2}}$ forming hyper multiplets, a localization computation yields [26]

$$
Z^{\mathrm{SUSY}}\left(b, \beta, m_{v}\right)=e^{-\beta E_{\text {susy }}\left(b, m_{v}\right)} \mathcal{I}\left(b, \beta, m_{v}\right),
$$

with

$$
\begin{aligned}
\mathcal{I}\left(b, \beta, m_{v}\right)=\frac{(p ; p)^{r_{G}}(q ; q)^{r_{G}}}{|W|} \int \mathrm{d}^{r_{G}} x & \prod_{\alpha_{+}}\left(\frac{\Gamma\left((p q)^{1 / 3} v z^{ \pm \alpha_{+}}\right)}{\Gamma\left(z^{ \pm \alpha_{+}}\right)}\right) \\
& \prod_{\chi} \prod_{\rho^{\chi \in \Delta_{\chi}}} \Gamma\left((p q)^{r_{\chi} / 2} v^{3\left(r_{\chi}-1\right) / 2} z^{\rho^{\chi}}\right),
\end{aligned}
$$

the $\mathcal{N}=2$ index of the SUSY gauge theory, and $E_{\text {susy }}\left(b, m_{v}\right)$ the corresponding Casimir polynomial, which can be obtained from (3.3) by substituting on its r.h.s.

$$
\omega \rightarrow \omega+\frac{3}{2} m_{v}
$$

A quick way to see why this shift is expected is to note that the argument of the chiralmultiplet gamma functions in (4.5) contain $(p q)^{r_{\chi} / 2} v^{3\left(r_{\chi}-1\right) / 2}=e^{i \beta \omega r_{\chi}} \cdot e^{i \beta m_{v}\left[3\left(r_{\chi}-1\right) / 2\right]}$, whereas if $m_{v}$ were zero we would only have $e^{i \beta \omega r_{\chi}}$. Consequently, to obtain the dependence of various quantities on $m_{v}$, we can start with their expression for when $m_{v}=0$, and replace in them every $\omega r_{\chi}$ with $\omega r_{\chi}+m_{v}\left[3\left(r_{\chi}-1\right) / 2\right]$. In particular, this amounts to replacing every $\left(r_{\chi}-1\right) \omega$ with $\left(r_{\chi}-1\right)\left[\omega+\frac{3}{2} m_{v}\right]$, which can be alternatively realized as a shift in $\omega$, as the prescription (4.6) indicates. A similar argument applies to the $\mathcal{N}=2$ vector multiplets.

The Hamiltonian route to the $\mathcal{N}=2$ index is via

$$
\mathcal{I}\left(b, \beta, m_{v}\right)=\operatorname{Tr}\left[(-1)^{F} e^{-\hat{\beta}\left(\Delta-2 j_{2}-\frac{3}{2} r\right)} p^{j_{1}+j_{2}+\frac{1}{2} r} q^{-j_{1}+j_{2}+\frac{1}{2} r} v^{\frac{3}{2} r-3 R_{\mathcal{N}=2}}\right] .
$$

The case $m_{v}=0$ corresponds to the $\mathcal{N}=1$ index, which we already know how to deal with. The new challenge is to find the dependence of the asymptotics on $m_{v}$.

First of all, the low-temperature asymptotics is found as in the previous section, and (assuming all $r_{\chi}$ are in $] 0,2[$ ) reads

$$
\mathcal{I}\left(b, \beta, m_{v}\right) \simeq 1 \Rightarrow Z^{\mathrm{SUSY}}\left(b, \beta, m_{v}\right) \simeq e^{-\beta E_{\text {susy }}\left(b, m_{v}\right)} \quad\left(\text { as } 1 / \beta \rightarrow 0, \text { with } b, m_{v} \text { fixed }\right) .
$$

To find the high-temperature asymptotics, we first use the estimates (3.8), (2.11), and (2.14) in the integrand of the $\mathcal{N}=2$ index. Proceeding as in the previous section, we find

$$
Z^{\mathcal{N}=2}\left(b, \beta, m_{v}\right) \approx \mathcal{I}\left(b, \beta, m_{v}\right) \approx\left(\frac{2 \pi}{\beta}\right)^{r_{G}} \int_{\mathfrak{h}_{c l}} \mathrm{~d}^{r_{G}} x e^{-\left[\mathcal{E}_{0}^{D K}\left(b, \beta, m_{v}\right)+V^{\mathrm{eff}}\left(\mathbf{x} ; b, \beta, m_{v}\right)\right]},
$$


with $\mathcal{E}_{0}^{D K}\left(b, \beta, m_{v}\right)$ a function easily obtainable from (3.10) by applying on its r.h.s. the substitution (4.6). The effective potential $V^{\mathrm{eff}}\left(\mathbf{x} ; b, \beta, m_{v}\right)$ can be obtained similarly, and reads

$$
V^{\mathrm{eff}}\left(\mathbf{x} ; b, \beta, m_{v}\right)=\frac{4 \pi^{2}}{\beta}\left(\frac{b+b^{-1}}{2}\right) L_{h}\left(\mathbf{x}, m_{v}\right)
$$

where we have defined the $\mathcal{N}=2$ Rains function as

$$
L_{h}\left(\mathbf{x}, m_{v}\right)=\left(1+\frac{3 m_{v}}{2 \omega}\right) L_{h}(\mathbf{x}) .
$$

Note that we have not included a phase $\Theta$ in (4.9), the way we did in the previous section. The reason is that we are assuming the hyper multiplets consist of pairs of chiral multiplets sitting in conjugate representations of the gauge group. In other words $Q_{h}=0$ for all the Lagrangian $\mathcal{N}=2$ theories of our interest.

Remarkably, according to (4.11), the effect of nonzero $m_{v}$ in $L_{h}\left(\mathbf{x}, m_{v}\right)$ is only a multiplicative overall factor. Assuming that $m_{v}$ is - just like $\omega$ - pure imaginary, we conclude that nonzero $m_{v}$ does not modify the locus of the high-temperature localization of the matrix-integral. We can thus apply the shift (4.6) in the asymptotics of the SUSY partition function in (3.30) to obtain

$$
\ln Z^{\mathcal{N}=2}\left(b, \beta, m_{v}\right)=i \frac{\pi^{2}}{3 \beta}\left(\omega+\frac{3}{2} m_{v}\right)\left(\operatorname{Tr} R+12 L_{h \min }\right)+\operatorname{dimh} \mathfrak{h}_{q u} \ln \left(\frac{2 \pi}{\beta}\right)+O\left(\beta^{0}\right) .
$$

\subsection{Asymptotics of the Schur partition function and the Schur index}

An immediate corollary is the asymptotics of the Schur partition function, defined by setting in the $\mathcal{N}=2$ partition function $m_{v}=\frac{\omega}{3}=\frac{i}{3}$ :

$$
\ln Z^{\operatorname{Schur}}(\beta)=-\frac{\pi^{2}}{2 \beta}\left(\operatorname{Tr} R+12 L_{h \text { min }}\right)+\operatorname{dimh}_{q u} \ln \left(\frac{2 \pi}{\beta}\right)+O\left(\beta^{0}\right) \quad(\text { as } \beta \rightarrow 0) .
$$

The Schur index $\mathcal{I}^{\text {Schur }}(\beta)$ is similarly defined by setting $m_{v}=\frac{\omega}{3}=\frac{i}{3}$ in the $\mathcal{N}=2$ index. The relation between $\mathcal{I}^{\text {Schur }}$ and $Z^{\text {Schur }}$ follows from (4.4) to be

$$
Z^{\text {Schur }}(\beta)=e^{-\beta c / 2} \mathcal{I}^{\text {Schur }}(\beta),
$$

where

$$
c:=\frac{1}{32}\left(9 \operatorname{Tr} R^{3}-5 \operatorname{Tr} R\right)
$$

is the $c$ central charge [58].

To the order shown in (4.13), the asymptotics of $\ln \mathcal{I}^{\operatorname{Schur}}(\beta)$ and $\ln Z^{\text {Schur }}(\beta)$ match; the difference is of course at order $\beta$.

When $L_{h}$ min $=0$, the leading asymptotics in (4.13) gives the Cardy-like piece noted recently in some examples by Buican and Nishinaka [22].

For theories whose Rains function is minimized only at the origin of $\mathfrak{h}_{c l}$, we can apply the shift (4.6) to (3.40), and obtain

$$
\ln \mathcal{I}\left(b, \beta, m_{v}\right) \sim i \frac{\pi^{2}}{3 \beta}\left(\omega+\frac{3}{2} m_{v}\right) \operatorname{Tr} R+\ln Z_{S^{3}}\left(b, m_{v}\right)+\beta E_{\text {susy }}\left(b, m_{v}\right), \quad(\text { as } \beta \rightarrow 0)
$$


with some $Z_{S^{3}}\left(b, m_{v}\right)$ which can be easily derived from (4.5). Upon setting $m_{v}=\frac{\omega}{3}=\frac{i}{3}$ in (4.16) we find

$$
\ln \mathcal{I}_{\text {Schur }}(\beta) \sim \frac{8 \pi^{2}}{\beta}(c-a)+\ln Z_{S^{3}}\left(b=1, m_{v}=i / 3\right)+\beta c / 2 \quad(\text { as } \beta \rightarrow 0),
$$

where

$$
a:=\frac{3}{32}\left(3 \operatorname{Tr} R^{3}-\operatorname{Tr} R\right)
$$

is the $a$ central charge [58].

Our preliminary (unpublished) results suggest that the asymptotic relation (4.17) also holds (with some $Z_{S^{3}}\left(b=1, m_{v}=i / 3\right)$ ) for all the non-Lagrangian $T_{N}$ SCFTs. The Schur index of these theories is given in [59].

\subsubsection{The Schur partition function of $\mathrm{SU}(N) \mathcal{N}=4 \mathrm{SYM}$}

As discussed in subsection 3.1, the Rains function of the $\mathcal{N}=4$ theory vanishes. Therefore $L_{h \min }=0$ and $\operatorname{dimh}_{q u}=N-1$. Since for this theory also $\operatorname{Tr} R=0$, (4.13) yields

$$
\ln Z_{\mathrm{SU}(N) \mathcal{N}=4}^{\mathrm{Schur}}(\beta)=(N-1) \ln \left(\frac{2 \pi}{\beta}\right)+O\left(\beta^{0}\right) \quad(\operatorname{as} \beta \rightarrow 0) .
$$

More precise asymptotics. We now improve (4.19) by reducing its error to $o(1)$. The reader not interested in the technical details of the derivation can skip to (4.23) and continue reading from there.

The starting point is the matrix-integral computing the Schur partition function

$$
Z_{\mathrm{SU}(N) \mathcal{N}=4}^{\mathrm{Schur}}(\beta)=e^{-\beta\left(N^{2}-1\right) / 8} \frac{(q ; q)^{2(N-1)}}{N !} \Gamma^{2(N-1)}\left(q^{1 / 2}\right) \int \mathrm{d}^{N-1} x \prod_{1 \leq i<j \leq N} \frac{\Gamma^{2}\left(q^{1 / 2}\left(z_{i} / z_{j}\right)^{ \pm 1}\right)}{\Gamma\left(\left(z_{i} / z_{j}\right)^{ \pm 1}\right)}
$$

with the integral over $x_{i} \in[-1 / 2,1 / 2]$, and $\prod_{i=1}^{N} z_{i}=1$. The estimate (3.53) guarantees that, outside an $\varepsilon_{1}$ neighborhood of $\mathcal{S}_{g}$, the integrand is well approximated by unity. Therefore

$$
Z_{\mathrm{SU}(N) \mathcal{N}=4}^{\mathrm{Schur}}(\beta)=\frac{(q ; q)^{2(N-1)}}{N !} \Gamma^{2(N-1)}\left(q^{1 / 2}\right)(1+o(1))
$$

The $o(1)$ error above comes from neglecting $i)$ the $e^{-\beta c / 2}$ prefactor; ii) the contribution to the integral from the $\varepsilon_{1}$ neighborhood of $\mathcal{S}_{g}$, where the estimate (3.53) does not apply, and the integrand differs from unity by some multiplicative factor of order one.

To write down the high-temperature asymptotics of (4.21) more explicitly, we need the following estimate [9]:

$$
\begin{aligned}
\ln \Gamma\left(q^{r} ; q, q\right) \sim & -\frac{\pi^{2}}{3 \beta}(r-1)+\left((r-1) \ln \left(1-e^{-2 \pi i r}\right)-\frac{1}{2 \pi i} L i_{2}\left(e^{-2 \pi i r}\right)+\frac{i \pi(r-1)^{2}}{2}-\frac{i \pi}{12}\right) \\
& +\beta\left(\frac{r^{3}}{6}-\frac{r^{2}}{2}+\frac{5 r}{12}-\frac{1}{12}\right) .
\end{aligned}
$$


Combining (4.21) and (4.22), and using $L i_{2}(-1)=-\pi^{2} / 12$, we find

$$
\ln Z_{\mathrm{SU}(N)}^{\mathrm{Schur}} \mathcal{N}=4(\beta)=(N-1) \ln \left(\frac{2 \pi}{\beta}\right)-(N-1) \ln 2-\ln N !+o(1)
$$

This asymptotic relation is confirmed in appendix B using a very different approach.

Interestingly, a comparison of the Schur partition function of the $\mathrm{SU}(2) \mathcal{N}=4$ theory in (4.20) and the SUSY partition function (with $p=q$ ) of SO(3) SQCD with two flavors in (3.49), reveals that the two precisely coincide. In fact, the $\mathcal{N}=2$ partition function of the $\mathrm{SU}(2) \mathcal{N}=4$ theory with $v=(p q)^{1 / 6}$, coincides with the SUSY partition function of $\mathrm{SO}(3) \mathrm{SQCD}$ with two flavors even when $p \neq q$. It would be nice to have a deeper understanding of this coincidence.

\subsection{The example of the non-Lagrangian $E_{6}$ SCFT}

For non-Lagrangian theories the $\mathcal{N}=2$ partition function can not be defined via pathintegration. Nonetheless, the $\mathcal{N}=2$ index is well-defined from the Hamiltonian perspective of (4.7). When the 't Hooft anomalies of the theory are known, one can then compute the $\mathcal{N}=2$ Bobev-Bullimore-Kim polynomial $E_{\text {susy }}\left(b, m_{v}\right)$, and define the $\mathcal{N}=2$ partition function via (4.4). This procedure can be done, for instance, for the $E_{6}$ SCFT [60], whose $\mathcal{N}=2$ index and $E_{\text {susy }}\left(b, m_{v}\right)$ are both known.

It turns out that our methods do not apply directly to the $\mathcal{N}=2$ partition function of the $E_{6}$ SCFT. We instead consider an equivariant deformation of the $\mathcal{N}=2$ partition function, which is computed by path-integration in presence of a real background $\mathrm{U}(1)_{w}$ gauge field $m_{w}$ along $S_{\beta}^{1}$, that couples a conserved $\mathrm{U}(1)$ flavor charge in the theory. We denote the resulting partition function by $Z_{E_{6}}^{\mathcal{N}=2}\left(b, \beta, m_{v} ; m_{w}\right)$. This equivariant partition function is related to an equivariant $\mathcal{N}=2 \operatorname{index} \mathcal{I}^{E_{6}}\left(b, \beta, m_{v} ; m_{w}\right)$, in which $w:=e^{i \beta m_{w}}$ plays the role of an additional fugacity for the $\mathrm{U}(1)_{w}$ charge. An equation similar to (4.4), but with an equivariant Bobev-Bullimore-Kim polynomial $E_{\text {susy }}\left(b, m_{v} ; m_{w}\right)$, mediates $Z_{E_{6}}^{\mathcal{N}=2}\left(b, \beta, m_{v} ; m_{w}\right)$ and $\mathcal{I}^{E_{6}}\left(b, \beta, m_{v} ; m_{w}\right)$. Explicitly [26]

$$
\begin{aligned}
E_{\mathrm{susy}}^{E_{6}}\left(b, m_{v} ; m_{w}\right)= & \frac{i}{6}\left(\frac{98}{27}\right)\left(\omega+\frac{3}{2} m_{v}\right)^{3}+i\left(\frac{b^{2}+b^{-2}}{24}\right)\left(-\frac{22}{3}\right)\left(\omega+\frac{3}{2} m_{v}\right) \\
& +\frac{i}{2}(4) m_{w}^{2}\left(\omega+\frac{3}{2} m_{v}\right)
\end{aligned}
$$

Note that the effect of nonzero $m_{v}$ is accounted for precisely by the shift (4.6). Setting $m_{w}=0$ and comparing with (3.3) reveals, for example, that $\operatorname{Tr} R^{3}=98 / 27$. The effect of nonzero equivariant parameters such as $m_{w}$ is easily obtained in general by shifting $R \omega$ in (3.3) to $R \omega+Q_{w} m_{w}$. The $m_{w}$-dependent terms in the equivariant Bobev-Bullimore-Kim polynomial then encode various ' $t$ Hooft anomalies associated to the $\mathrm{U}(1)_{w}$ current. The second line of (4.24), for instance, indicates that $\operatorname{Tr} R Q_{w}^{2}=4$. 
The $\mathcal{N}=2$ index of the $E_{6} \mathrm{SCFT}$ (also known as the $T_{3}$ theory) is computed in [61], and is given by

$$
\begin{aligned}
\mathcal{I}^{E_{6}}\left(b, \beta, m_{v} ; m_{w}\right)= & \frac{(p ; p)(q ; q)}{2 \Gamma\left((p q)^{1 / 3} v w^{ \pm 2}\right) \Gamma\left((p q)^{-2 / 3} v\right)} \int_{-1 / 2}^{1 / 2} \mathrm{~d} x_{s} \frac{\Gamma\left((p q)^{-1 / 3} v^{1 / 2} w^{ \pm 1} s^{ \pm 1}\right)}{\Gamma\left(s^{ \pm 2}\right)} \hat{\mathcal{I}}(s) \\
& +\frac{1}{2} \frac{\Gamma\left(w^{-2}\right)}{\Gamma\left((p q)^{1 / 3} v w^{-2}\right)}\left(\hat{\mathcal{I}}\left(s=(p q)^{-1 / 3} v^{1 / 2} w\right)+\hat{\mathcal{I}}\left(s=(p q)^{1 / 3} v^{-1 / 2} w^{-1}\right)\right) \\
& +\frac{1}{2} \frac{\Gamma\left(w^{2}\right)}{\Gamma\left((p q)^{1 / 3} v w^{2}\right)}\left(\hat{\mathcal{I}}\left(s=(p q)^{-1 / 3} v^{1 / 2} w^{-1}\right)+\hat{\mathcal{I}}\left(s=(p q)^{1 / 3} v^{-1 / 2} w\right)\right),
\end{aligned}
$$

where

$$
\begin{aligned}
\hat{\mathcal{I}}(s)=\frac{(p ; p)^{2}(q ; q)^{2}}{3 !} \Gamma\left((p q)^{1 / 3} v\right)^{2} \int \mathrm{d}^{2} x & \left(\prod_{1 \leq i<j \leq 3} \frac{\Gamma\left((p q)^{1 / 3} v\left(z_{i} / z_{j}\right)^{ \pm 1}\right)}{\Gamma\left(\left(z_{i} / z_{j}\right)^{ \pm 1}\right)}\right) \\
& \left(\prod_{i=1}^{3} \Gamma\left((p q)^{1 / 3} v^{-1 / 2}\left(s^{-1 / 3} z_{i}\right)^{ \pm 1}\right)\right)^{2},
\end{aligned}
$$

with the integral over the square $-1 / 2 \leq x_{1}, x_{2} \leq 1 / 2$. The parameters are related via $s=e^{2 \pi i x_{s}}, z_{i}=e^{2 \pi i x_{i}}$, and are constrained to satisfy $\sum_{i=1}^{3} x_{i} \in \mathbb{Z}$.

Luckily, the expression (4.25) involves the Pochhammer symbols and elliptic gamma functions that we are already familiar with. The method of Rains hence applies immediately.

As $\beta \rightarrow 0$, the integrals in each of the three lines of (4.25) take the form (4.9), with some effective potentials and associated Rains functions that can be easily obtained. For example, the Rains function associated to the integral on the first line is ${ }^{7}$

$$
\begin{aligned}
L_{h}^{1^{s t}} \text { line }\left(x_{1}, x_{2}, x_{s}\right)= & 2\left(1+\frac{2}{3}\right) \vartheta\left(x_{s}\right)-\vartheta\left(2 x_{s}\right) \\
& +(1-2 / 3-1) \sum_{i<j} \vartheta\left(x_{i}-x_{j}\right)+6\left(1-\frac{2}{3}\right) \sum_{i} \vartheta\left(x_{i}-x_{s} / 3\right),
\end{aligned}
$$

with the second line of the above function coming from the integrand of $\hat{\mathcal{I}}(s)$. Rains's generalized triangle inequality (2.20) then implies that $L_{h}^{1^{s t}}$ line is minimized at $x_{1}=x_{2}=$ $x_{s}=0$. We can thus prune the integral tails, use our central estimate (2.16), and then employ the inequality (2.23) to ensure that tails completion is safe. The evaluation of the asymptotics thus proceeds similarly to the cases in subsection 3.1. Note that the nonzero real parameter $m_{w}$ leads to a nonzero phase $\Theta$ in (3.9), even though the analog of $Q_{h}$ for the integrand of (4.25) vanishes. But as in subsection 3.1 the nonzero phase does not present an obstacle to our analysis, because the Rains function is minimized only at the origin.

\footnotetext{
${ }^{7}$ Note that to write the first term in the following Rains function, we are applying (2.11) with $r=-2 / 3$. This extrapolation of $\left(2.11\right.$ ) can be justified (when $b, b^{-1} \neq \sqrt{2}$ ) by an argument similar to the one in appendix A. In fact the derivation of (2.11) in appendix A indicates that constraining $r$ to the range ]0,2[ is too conservative. Similarly, (2.16) has to be extrapolated to obtain (4.28).
} 
All in all, we find the asymptotics

$$
\ln \mathcal{I}^{E_{6}}\left(b, \beta, m_{v} ; m_{w}\right) \sim i \frac{\pi^{2}}{3 \beta}(\operatorname{Tr} R)\left(\omega+\frac{3}{2} m_{v}\right)+\ln Z_{3 d}^{E_{6}}\left(b, m_{v} ; m_{w}\right)+\beta E_{\mathrm{susy}}^{E_{6}}\left(b, m_{v} ; m_{w}\right),
$$

with $\operatorname{Tr} R=-22 / 3$, and with some $Z_{3 d}^{E_{6}}\left(b, m_{v} ; m_{w}\right)$ whose derivation we omit. From (4.4) we then conclude

$$
\ln Z_{E_{6}}^{\mathcal{N}=2}\left(b, \beta, m_{v} ; m_{w}\right) \sim i \frac{\pi^{2}}{3 \beta}(\operatorname{Tr} R)\left(\omega+\frac{3}{2} m_{v}\right)+\ln Z_{3 d}^{E_{6}}\left(b, m_{v} ; m_{w}\right), \quad(\text { as } \beta \rightarrow 0)
$$

just as if the $E_{6} \mathrm{SCFT}$ was a Lagrangian $\mathcal{N}=2$ theory with finite $Z_{S^{3}}$.

\section{Discussion}

In this work we have studied the SUSY partition function of $4 \mathrm{~d}$ supersymmetric gauge theories with a $\mathrm{U}(1)_{R}$ symmetry, and with a (compact) semi-simple gauge group. More precisely, we have also assumed the R-charges of the chiral multiplets to be inside the interval $\left.^{8}\right] 0,2$ [, and we have taken the cancelation of the following anomalies for granted:

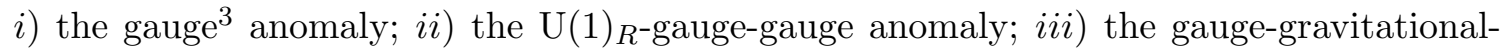
gravitational anomaly; and $i v$ ) the gauge- $\mathrm{U}(1)_{R^{-}} \mathrm{U}(1)_{R}$ anomaly. (These anomaly cancelation conditions are related to the so-called total ellipticity property of the associated elliptic hypergeometric integrals [31].)

A major role in our analysis is played by the Rains function $L_{h}\left(x_{1}, \ldots, x_{r_{G}}\right)$ of the SUSY gauge theory, defined in eq. (3.14) (see (2.12) for the definition of the function $\vartheta$ appearing in $L_{h}$ ). According to eq. (3.11), $L_{h}$ is proportional to $V^{\text {eff }}$.

Another important role was played above by the function $Q_{h}\left(x_{1}, \ldots, x_{r_{G}}\right)$ of the SUSY gauge theory, defined in eq. (3.13) (see (2.13) for the definition of the function $\kappa$ appearing in $Q_{h}$ ). Only theories with chiral matter content may have nonzero $Q_{h}$. Such nonzero $Q_{h}$ can make the high-temperature analysis of the SUSY partition function difficult.

Let us now recapitulate some of our main findings, and then move on to exploring the unresolved problems and open directions related to the subject of this work.

- It is sometimes said in the literature that "as $\beta \rightarrow 0$, the SUSY partition function of a $4 \mathrm{~d}$ theory reduces (after its divergent Cardy-like piece is stripped off) to the squashed-three-sphere partition function of the $3 \mathrm{~d}$ theory obtained by reducing the $4 \mathrm{~d}$ theory on $S_{\beta}^{1}$ ". As already emphasized in $[8,35]$, this statement is not generally true. In section 3 , we have obtained the condition under which the above statement is true in a SUSY gauge theory with a semi-simple gauge group: the Rains function

\footnotetext{
${ }^{8}$ Otherwise, it seems like the SUSY partition function would be ill-defined. On $S^{3} \times S^{1}$, the scalars inside a chiral multiplet have a curvature coupling, which gives their Kaluza-Klein zero-modes a mass. This mass would become non-positive (yielding a non-compact Higgs branch, or a tachyonic direction) if the R-charge of the multiplet does not belong to $] 0,2[$. Nonetheless, it may be possible to use meromorphic continuation (of the path-integral, or of the Romelsberger prescription [62]) to consistently assign SUSY partition functions to theories containing chiral multiplets with $r \notin] 0,2[$.
} 
of the $4 \mathrm{~d}$ theory must have a unique minimum at the origin of $\mathfrak{h}_{c l}$ (corresponding to $\left.x_{1}=\cdots=x_{r_{G}}=0\right)$. In particular, this condition is satisfied in all the $\mathrm{SU}(N) A D E$ SQCD theories discussed in [25, 63], and also the $\mathrm{Sp}(2 N)$ SQCD theories discussed in $[11]$.

- In $[9,37]$ prescriptions were put forward for extracting the central charges of a finite- $N$ 4d SCFT from its superconformal index. The example of the SO(3) SQCD with two flavors, that we studied in section 3 , shows that the finite- $N$ prescriptions of $[9,37]$ are not valid in general. On the other hand, the said prescriptions can be applied (for extracting $c$ and $a$ as in (4.15) and (4.18)) successfully to SUSY gauge theories with a semi-simple gauge group, with non-chiral matter content (hence $Q_{h}=0$ ), and with $\operatorname{dimh}_{q u}=0$. (In fact all the 't Hooft anomalies of such theories can be extracted from the high-temperature asymptotics of their equivariant Romelsberger in$\operatorname{dex} \mathcal{I}\left(b, \beta ; m_{a}\right)$; see the comments below (3.82).) Moreover, even nonzero $Q_{h}$ (arising from chiral matter content) does not present an obstruction to the said prescriptions if the Rains function of the theory has a unique minimum at the origin of $\mathfrak{h}_{c l}$ (see the comments below (3.40)).

- We have shown that the leading high-temperature asymptotics of $\ln Z^{\mathrm{SUSY}}(\beta)$ is not universal for SUSY gauge theories with a semi-simple gauge group, in the following sense. If the Rains function of the theory is not minimized at the origin of $\mathfrak{h}_{c l}$, the distance between $\mathfrak{h}_{q u}$ and the origin can serve as an order parameter for labeling the infinite-temperature phase of the theory on $S_{b}^{3} \times S_{\beta}^{1}$. [Note that at any finite temperature, a finite- $N$ gauge theory on $S_{b}^{3} \times S_{\beta}^{1}$ can not be assigned a phase, because the spatial manifold of the theory is compact. In the infinite-temperature limit, however, a phase emerges. The possibility of emergence of a thermodynamic ensemble in the high-temperature limit of a relativistic finite-volume system can be most easily understood in free QFTs; the Fock space of a free QFT becomes populated without a bound as $\beta \rightarrow 0$.] If this order parameter is nonzero, the leading high-temperature asymptotics of $\ln Z^{\mathrm{SUSY}}(\beta)$ may differ - and would certainly differ if the theory is non-chiral — from the generic Cardy-like asymptotics in (1.1).

The remarks in the last bullet point above suggest the following interpretation for the asymptotic relations we found in the ISS and the $\mathrm{BCI}_{5}$ models. Let's begin with the $\mathrm{BCI}_{5}$ theory. Figure 9 indicates that this theory has an infinite-temperature phase which partially breaks the gauge group $\mathrm{SO}(5)$. Indeed the expression for $Y_{S^{3}}^{B C I_{5}}(b)$ in (3.90) suggests that in this Higgsed phase, the 3d theory effectively consists of an $\mathrm{SO}(3)$ vector multiplet with a chiral matter multiplet in the five-dimensional representation, and an SQED theory. For the ISS model, the expression for $Y_{S^{3}}^{\mathrm{ISS}}(b)$ in (3.81) suggests again a Higgsed phase at infinite temperature, this time with only an SQED effective $3 \mathrm{~d}$ theory. (It might be possible to interpret the exponential function in the integrand of (3.81) as an induced FI parameter.) 


\subsection{Open problems}

We have not treated theories with nonzero $Q_{h}$ in full generality. The following problem is thus the most important loose end of the present work.

Problem 1) Restricting still to SUSY gauge theories with a semi-simple gauge group, find a general expression similar to (3.30), that is valid for theories with $Q_{h} \neq 0$.

A related puzzle is the following.

Problem 1.1) Find a SUSY gauge theory with a semi-simple gauge group, in which $Q_{h}$ is nonzero on the minimum set of $L_{h}$. (Or prove that such a theory does not exist.)

Even focusing on theories with $Q_{h}=0$, we have not been able to clarify some of the intriguing phenomena we observed in our explicit examples. For instance, our case by case investigation suggests that $\operatorname{Tr} R>0$ when $L_{h}$ is not positive semi-definite (the ISS and the $\mathrm{BCI}_{\geq 5}$ models), and that $\operatorname{Tr} R=0$ when $L_{h}$ vanishes identically (the $\mathrm{SO}(3) \mathrm{SQCD}$ and the $\mathcal{N}=4 \mathrm{SYM}$ ). It is highly desirable to know if these correlations are general or not. We can phrase this as follows.

Problem 2) Is there a general correlation between the sign of $\operatorname{Tr} R$ in a SUSY gauge theory with a semi-simple gauge group, and the sign of the theory's $L_{h}$ min?

A possibly related problem is the connection between the finiteness of $Z_{S^{3}}(b)$ and the validity of the Di Pietro-Komargodski formula. As discussed in the introduction, we suspect (but have not been able to show) that all theories with finite $Z_{S^{3}}(b)$ satisfy the Di Pietro-Komargodski formula. The following problem phrases the question in terms of the functions $L_{h}$ and $\tilde{L}_{S^{3}}$ (see eq. (3.20) for the definition of $\tilde{L}_{S^{3}}$ ).

Problem 3) Prove (or disprove) that in a SUSY gauge theory with a semi-simple gauge group, if the function $\tilde{L}_{S^{3}}$ (and thus $L_{h}$ ) is strictly positive in some punctured neighborhood of the origin, then $L_{h}$ is positive semi-definite.

Another important direction for extending the present work is the following.

Problem 4) Extend the results of the present paper to SUSY gauge theories with a compact gauge group.

The added difficulty would of course be in analyzing the extra U(1) factors in the gauge group.

\subsection{Two new simple tests of supersymmetric dualities}

Dual QFTs must have equal partition functions. As a trivial corollary, the hightemperature asymptotics of the SUSY partition functions of dual 4d SUSY QFTs must match.

Assume now that both sides of the duality are 4d SUSY gauge theories (with a $\mathrm{U}(1)_{R}$ symmetry, and free of various harmful anomalies) with a semi-simple gauge group, and with $Q_{h}=0$. The relation (3.30) then yields two quantities to be matched between the theories: 
$L_{h \text { min }}$ and $\operatorname{dimh} \mathfrak{h}_{q u}$. Comparison of $L_{h \text { min }}$ can rule out for instance the confinement scenario for the SU(2) ISS model: on the gauge theory (UV) side, as discussed in subsection 3.3, we have $L_{h}$ min $=-2 / 15$, while on the mesonic (IR) $\operatorname{side}^{9}$ we have no gauge group and thus $L_{h}=0$.

As another example, consider the recent $E_{7} \mathrm{SQCD}$ duality of $[25,65]$. In that case a direct examination reveals that $L_{h \text { min }}=\operatorname{dim} \mathfrak{h}_{q u}=0$, both on the electric and the magnetic side. Their proposal hence passes both our tests.

Our numerical investigation indicates that the magnetic Pouliot theory with $N_{f}=7$ and its electric dual [50] also both have $L_{h} \min =\operatorname{dimh}_{q u}=0$, and thus their duality passes our tests. Note that on the magnetic side, since the Rains function is minimized only at the origin of $\mathfrak{h}_{c l}$, the discussion of subsection 3.1 applies, and therefore the nonzero $Q_{h}$ does not present an obstruction to performing the tests in this case.

The case of the SCFTs with $c<a$ (namely the IR fixed points of the ISS model, and the $\mathrm{BCI}_{2 N+1}$ model with $1<N<5$ ) is particularly interesting. A dual description for these theories is currently lacking. Our results for $L_{h \text { min }}$ and $\operatorname{dimh} \mathfrak{h}_{q u}$ on the electric side might help to test future proposals for magnetic duals of these theories.

\subsection{Holography and the asymptotics of $4 \mathrm{~d}$ superconformal indices}

Studying the high-temperature asymptotics of the large- $N$ limit of the superconformal indices of 4d SCFTs has already proven fruitful for holography. It has led to a rather general solution to the problem of Holographic Weyl Anomaly in the traditional $\mathrm{AdS}_{5} / \mathrm{CFT}_{4}$ scenarios $[9,37]$. More precisely, at the leading order $\left(O\left(N^{2}\right)\right)$, the holographic Weyl anomaly in $\mathrm{AdS}_{5} / \mathrm{CFT}_{4}$ was addressed by Henningson-Skenderis [66] and Gubser [67] back in 1998. But in the traditional scenarios, with $\mathrm{AdS}_{5}$ times a toric Sasaki-Einstein 5-manifold ( $\left.\mathrm{SE}_{5}\right)$ on the gravity side, the anomaly has a subleading $O\left(N^{0}\right)$ piece whose AdS/CFT matching was open in the general case until the works [9, 37, 68]; in [37] the matching of the subleading piece was established (crucially relying on results of [68] and [69]) for the cases where the toric $\mathrm{SE}_{5}$ is smooth and the SCFT does not have matter in the adjoint representation of the gauge group(s); in [9] the matching was shown for the general case, assuming $i$ ) that the boundary single-trace index and the bulk single-particle index match, and $i i$ ) that an (essentially combinatorial) conjecture proposed and supported in [70] is valid.

In the present paper we analyzed the high-temperature asymptotics of the Romelsberger indices of various gauge theories at finite $N$. The finite- $N$ indices of holographic SCFTs are expected to encode information about micro-states of the supersymmetric Giant Gravitons of the dual string theories [7]. Take for instance the $\operatorname{SU}(N) \mathcal{N}=4 \mathrm{SYM}$.

\footnotetext{
${ }^{9}$ Following [56], we are assuming that a SUSY partition function can be consistently assigned to the proposed IR theory, even though the IR chiral multiplet would have R-charge $12 / 5 \notin] 0,2[$. This assignment requires an analytic continuation of the kind mentioned in footnote 8 . The duality test in [56] can then be thought of as comparing the low-temperature asymptotics of the supposedly dual SUSY partition functions; the low-temperature test goes beyond ' $t$ Hooft anomaly matching in that case because on the IR side the relation (3.7) and the comments below it do not apply. See [64] for an alternative take on this problem. I thank L. Di Pietro and Z. Komargodski for correspondence on this point, and for explaining to me related subtleties that were overlooked in an early draft of the present paper.
} 
One of the novel results of the present paper is the following high-temperature asymptotics for the superconformal index of this theory (see eqs. (3.67) and (3.68)):

$$
\mathcal{I}(b=1, \beta)=\sum_{\text {operators }}(-1)^{F} e^{-\beta\left(\Delta-\frac{1}{2} r\right)} \approx\left(\frac{1}{\beta}\right)^{N-1} .
$$

The above canonical relation can be transformed to the micro-canonical ensemble to yield the asymptotic (fermion-number weighted) degeneracy of the protected high-energy operators in the $\mathcal{N}=4$ theory:

$$
N(E) \approx E^{N-2},
$$

with $E=\Delta-r / 2$. This result should presumably be reproduced by geometric quantization of the 1/16 BPS Giant Gravitons of IIB theory on $\mathrm{AdS}_{5} \times S^{5}$, along the lines of [71]. It would be interesting to see if this expectation pans out.

\subsection{Crossed channel: quantum Coulomb branch dynamics on $R^{3} \times S^{1}$}

Take a $4 \mathrm{~d} \mathcal{N}=1$ SUSY gauge theory with a U(1) R-symmetry, and with a semi-simple gauge group. Its SUSY partition function $Z^{\mathrm{SUSY}}(b, \beta)$ was so far defined by a path-integral on $S_{b}^{3} \times S_{\beta}^{1}$, with $S_{b}^{3}$ the unit-radius squashed three-sphere. We now replace the $S_{b}^{3}$ with the round three-sphere $S_{r_{3}}^{3}$ of arbitrary radius $r_{3}>0$. The path-integral on the new space gives $Z^{\mathrm{SUSY}}\left(\beta ; r_{3}\right)=Z^{\mathrm{SUSY}}\left(b=1, \beta / r_{3}\right)$; i.e. the resulting partition function only depends on the ratio $\beta / r_{3}[5]$. Thus, as far as $Z^{\mathrm{SUSY}}\left(\beta ; r_{3}\right)$ is concerned, shrinking the $S^{1}$ is equivalent to decompactifying the $S^{3}$. We hence fix $\beta$, and send $r_{3}$ to infinity. In this limit we expect the unlifted zero-modes on $S_{r_{3}}^{3} \times S_{\beta}^{1}$ to roughly correspond to the quantum zero-modes on $R^{3} \times S^{1}$. Therefore at high temperatures the unlifted holonomies of the theory on $S_{r_{3}}^{3} \times S_{\beta}^{1}$ should be in correspondence with (a real section of) the quantum Coulomb branch of the $3 \mathrm{~d} \mathcal{N}=2$ theory obtained from compactifying the $4 \mathrm{~d}$ theory on the circle of $R^{3} \times S^{1}$. In particular, we expect $\operatorname{dim} \mathfrak{h}_{q u}$ to be equal to the (complex-) dimension of the quantum Coulomb branch of the $3 \mathrm{~d}$ theory. (Recall that the Coulomb branch of the $3 \mathrm{~d}$ theory consists not just of the holonomies around the $S^{1}$, but also of the dual $3 \mathrm{~d}$ photons; hence our references above to "a real section" and "complex-dimension".)

We do not expect to recover the $R^{3} \times S^{1}$ Higgs branch from the zero-modes on $S_{r_{3}}^{3} \times S_{\beta}^{1}$ : for any (arbitrarily small) curvature on the $S^{3}$, curvature couplings presumably lift the Higgs-type zero-modes on $S_{r_{3}}^{3} \times S_{\beta}^{1}$.

From the point of view of $R^{3} \times S^{1}$, picking one of the $R^{3}$ directions as time, ${ }^{10}$ we can relate $\mathcal{E}_{0}^{D K}$ to the Casimir energy associated to the spatial manifold $R^{2} \times S^{1}$ : we reintroduce $r_{3}$ in $\mathcal{E}_{0}^{D K}$ (by replacing its $\beta$ with $\beta / r_{3}$ ), set in it $b=1$, interpret $\tilde{\beta}:=2 \pi r_{3}$ as the circumference of the crossed channel thermal circle, and write

$$
\mathcal{E}_{0}^{D K}\left(\beta ; r_{3}\right)=\tilde{\beta} E_{0}^{R^{2} \times S^{1}}(\beta), \quad \text { with } \quad E_{0}^{R^{2} \times S^{1}}(\beta)=\frac{\pi}{6 \beta} \operatorname{Tr} R .
$$

\footnotetext{
${ }^{10}$ The following discussion is in the spirit of the arguments in [72], though our treatment is not as precise. We are approaching $R^{3}$ from $S^{3}$, rather than from $T^{3}$ (as in [72]). While on $T^{3}$ each of the circles can be picked as the time direction, picking a time direction along the $S^{3}$ makes the spatial sections time-dependent, rendering our arguments in the paragraph of this footnote somewhat hand-wavy. I thank E. Shaghoulian for several helpful conversations related to the subject of the present subsection.
} 
Now $E_{0}^{R^{2} \times S^{1}}(\beta)$ admits an interpretation as the Casimir energy associated to the spatial $R^{2} \times S_{\beta}^{1}$. Similarly, resurrecting the $r_{3}$ in $V^{\text {eff }}$, and setting in it $b=1$, we obtain what can be loosely regarded as $\tilde{\beta}$ times the quantum effective potential on (a real section of) the crossed channel Coulomb branch. From this perspective, the two tests we advocated in subsection 5.2 would not really be new, but would correspond to the comparison of low-energy properties on $R^{3} \times S^{1}$.

The discussion in the previous three paragraphs is rather intuitive, and should be considered suggestive at best. It is desirable to have it made more precise. Nevertheless, in the examples of the $\mathrm{SU}(N), \mathrm{Sp}(2 N)$, and $\mathrm{SO}(2 N+1) \mathrm{SQCD}$ theories, and the $\mathrm{SU}(N)$ $\mathcal{N}=4 \mathrm{SYM}$, we see that (upon quotienting by the Weyl group) $\mathfrak{h}_{q u}$ does indeed resemble (a real section of) the $R^{3} \times S^{1}$ quantum Coulomb branch; see [35, 36] and [73]. We therefore conjecture that the relation between $\mathfrak{h}_{q u}$ and the unlifted Coulomb branch on $R^{3} \times S^{1}$ continues to remain valid, at least for all the theories with a positive semi-definite Rains function. In particular, we predict that, when placed on $R^{3} \times S^{1}$, all the $\mathrm{SU}(N) A D E$ SQCD and the Pouliot theories (in the appropriate range of their parameters such that all their $r_{\chi}$ are in ]0,2[) have no quantum Coulomb branch, and the $\mathbb{Z}_{2}$ and $\mathbb{Z}_{3}$ orbifolds of the $\mathrm{SU}(N) \mathcal{N}=4$ theory have an $(N-1)$-dimensional unlifted Coulomb branch.

For theories whose Rains function is not positive semi-definite, on the other hand, it seems like this connection with $R^{3} \times S^{1}$ fails. The Rains function of the SU(2) ISS model does not have a flat direction, and appears to suggest a Higgs vacuum for the theory on $R^{3} \times S^{1}$. However, the study of [55] indicates that this theory possesses an unlifted Coulomb branch on $R^{3} \times S^{1}$, and in particular does not necessarily break the gauge group at low energies. It would be nice to understand if this conflict is only a manifestation of the sloppiness of our intuitive arguments above, or it has a more interesting origin.

\section{Acknowledgments}

The project reported on here was a direct outcome of the author's collaborations and discussions with Finn Larsen, Jim Liu, and Phil Szepietowski, whose ideas, help, and feedback have contributed to this work at various levels and various stages. I am grateful to them, as well as to C. Beem, J. Bourdier, N. Drukker, H. Elvang, J. Felix, A. Gadde, D. Mayerson, J. McGreevy, D. Poland, S. Razamat, C. Uhlemann, and especially F. Bouya, S. Chapman, K. Intriligator, G. Knodel, U. Kol, Y. Nakayama, K. Ohmori, L. Pando Zayas, E. Shaghoulian, and Jaewon Song for helpful conversations and correspondences related to the subject matter of this paper. I am particularly indebted to Peter Miller and Eric Rains for valuable discussions on the asymptotic analysis used in this work. I also thank L. Di Pietro and Z. Komargodski for their insightful comments and helpful feedback on a draft of this paper. The plots in this paper are all produced by Mathematica. This project was supported partly by the physics department at University of Michigan, and partly by the US Department of Energy under grant DE-SC0007859.

Finally, my profound thanks go to A. Peters for all the support, encouragement, and inspiration I have received from her while this work was in progress. This paper is dedicated to her. 


\section{A Derivation of the elliptic gamma function estimates}

Define the non-compact quantum dilogarithm $\psi_{b}$ (cf. the function $e_{b}(x)$ in [74]; $\psi_{b}(x)=$ $\left.e_{b}(-i x)\right)$ via

$$
\psi_{b}(x):=e^{-i \pi x^{2} / 2+i \pi\left(b^{2}+b^{-2}\right) / 24} \Gamma_{h}\left(i x+\omega ; \omega_{1}, \omega_{2}\right),
$$

where

$$
\omega_{1}:=i b, \quad \omega_{2}:=i b^{-1}, \quad \text { and } \quad \omega:=\left(\omega_{1}+\omega_{2}\right) / 2 .
$$

For generic choice of $b$, the zeros of $\psi_{b}(x)^{ \pm 1}$ are of first order, and lie at $\pm\left(\left(b+b^{-1}\right) / 2+\right.$ $\left.b \mathbb{Z}^{\geq 0}+b^{-1} \mathbb{Z}^{\geq 0}\right)$. Upon setting $b=1$ we get the function $\psi(x)$ of [75]; i.e. $\psi_{b=1}(x)=\psi(x)$.

From the asymptotics of the hyperbolic gamma function (see e.g. [13]), it follows that for fixed $\operatorname{Re}(x)$ and fixed $b>0$

$$
\ln \psi_{b}(x) \sim 0, \quad(\text { as } \beta \rightarrow 0, \text { for } \operatorname{Im}(x)=-1 / \beta)
$$

with a transcendentally small error, of the type $e^{-1 / \beta}$.

An identity due to Narukawa [76] implies (see also appendix A of [9])

$$
\Gamma(x ; \sigma, \tau):=e^{2 i \pi Q_{+}(x ; \sigma, \tau)} \psi_{b}\left(-\frac{2 \pi i x}{\beta}-\frac{b+b^{-1}}{2}\right) \prod_{n=1}^{\infty} \frac{\psi_{b}\left(-\frac{2 \pi i n}{\beta}-\frac{2 \pi i x}{\beta}-\frac{b+b^{-1}}{2}\right)}{\psi_{b}\left(-\frac{2 \pi i n}{\beta}+\frac{2 \pi i x}{\beta}+\frac{b+b^{-1}}{2}\right)},
$$

where

$$
\begin{aligned}
Q_{+}(x ; \sigma, \tau)= & -\frac{x^{3}}{6 \tau \sigma}+\frac{\tau+\sigma+1}{4 \tau \sigma} x^{2}-\frac{\tau^{2}+\sigma^{2}+3 \tau \sigma+3 \tau+3 \sigma+1}{12 \tau \sigma} x \\
& +\frac{1}{24}(\tau+\sigma+1)\left(1+\tau^{-1}+\sigma^{-1}\right) .
\end{aligned}
$$

The two relations (A.3) and (A.4) immediately imply (3.53). Moreover, the three relations (A.1), (A.3), and (A.4) imply (2.16) and (2.18).

To derive (2.11) we need the following fact: for fixed $r \in] 0,2[$ and fixed $b>0$, as $\beta \rightarrow 0$ the function $\ln \psi_{b}\left(-\frac{2 \pi i\{x\}}{\beta}+(r-1) \frac{b+b^{-1}}{2}\right)$ is uniformly bounded over $(x \in) \mathbb{R}$. It suffices of course to establish this fact in the "fundamental domain" $x \in[0,1[$. To obtain the uniform bound, divide this interval into $\left[0, N_{0} \beta\right]$ and $\left[N_{0} \beta, 1\right.$ [, with $N_{0}$ chosen as follows. Since $\psi_{b}\left(-2 \pi i N+(r-1) \frac{b+b^{-1}}{2}\right) \rightarrow 1$ as $N \rightarrow \infty$, there is a large enough $N_{0}$, so that for all $N>N_{0}$ we have $\psi_{b}\left(-2 \pi i N+(r-1) \frac{b+b^{-1}}{2}\right) \approx 1$, with an error of say .1. With this choice of $N_{0}$ it is clear that $\ln \psi_{b}\left(-\frac{2 \pi i x}{\beta}+(r-1) \frac{b+b^{-1}}{2}\right)$ is uniformly bounded over $\left[N_{0} \beta, 1[\right.$ (for all $\beta$ smaller than $\left.1 / N_{0}\right)$. On the other hand, since $\ln \psi_{b}\left(-2 \pi i x+(r-1) \frac{b+b^{-1}}{2}\right.$ ) is continuous, it is guaranteed to be uniformly bounded on the compact domain $\left[0, N_{0}\right]$; rescaling $x \mapsto \frac{x}{\beta}$ this implies the uniform bound on $\ln \psi_{b}\left(-\frac{2 \pi i x}{\beta}+(r-1) \frac{b+b^{-1}}{2}\right)$ over $\left[0, N_{0} \beta\right]$, and we are done. Note that for $\ln \psi_{b}\left(-\frac{2 \pi i\{x\}}{\beta}+(r-1) \frac{b+b^{-1}}{2}\right)$ to not diverge at $x \in \mathbb{Z}$, we need $r\left(\frac{b+b^{-1}}{2}\right) \notin b \mathbb{Z}^{\leq 0}+b^{-1} \mathbb{Z}^{\leq 0}$ and $(r-2)\left(\frac{b+b^{-1}}{2}\right) \notin b \mathbb{Z}^{\geq 0}+b^{-1} \mathbb{Z}^{\geq 0}$; our constraint $\left.r \in\right] 0,2[$ takes care of these.

To obtain (2.14), we can apply the argument of the previous paragraph, except that we do not get the uniform bound on $\left[0, N_{0} \beta\right]$ : our "continuous function with a compact support" argument fails when $r=0$, because $\psi_{b}\left(-\frac{2 \pi i\{x\}}{\beta}-\frac{b+b^{-1}}{2}\right)$ diverges at $x \in \mathbb{Z}$. This is why (2.14) applies uniformly only on $x \in \mathbb{R} \backslash \mathbb{Z}^{(\beta)}$, with $\mathbb{Z}^{(\beta)}$ an $O(\beta)$ neighborhood of $\mathbb{Z}$. 


\section{B Asymptotics of the Schur partition function of the $\mathcal{N}=4$ theory}

The Schur partition function of the $\mathcal{N}=4$ theory is exceptionally well under control, because of its connection with the partition function of a free-fermion system on a circle. Employing this connection, expressions for the Schur index of the $\mathcal{N}=4$ theory were obtained in [7], that we asymptotically analyze in this appendix. We write down the all-orders small- $\beta$ expansion of the log of this partition function.

Recall that the Rains function of the $\mathcal{N}=4$ theory vanishes, and hence for this theory $\operatorname{dimh} \mathfrak{h}_{q u}=\operatorname{dimh} \mathfrak{h}_{c l}$, which when the gauge group is $\mathrm{SU}(N)$ equals $N-1$. In the body of the paper we were not able to obtain all-orders asymptotics for the partition functions of theories with $\operatorname{dimh}_{q u}>0$. The partition function studied in this appendix is the only example with $\operatorname{dimh}_{q u}>0$ for which we can write down all-orders asymptotics.

Before spelling out the said partition function, we introduce the mathematical technique required for its asymptotic analysis. This technique is explained by Zagier in [29], but its proof was omitted there. We now present the method, outline its proof (mirroring a similar one in [29]), and along the way fix some typos in [29].

Consider a real function $G(\beta)$ defined in terms of another real function $f(\beta)$ as

$$
G(\beta)=\sum_{m=0}^{\infty} f((m+a) \beta),
$$

with some $a \in[0,1[$. Assume that $f(\beta)$ has the small- $\beta$ asymptotic development

$$
f(\beta) \sim \sum_{n=0}^{\infty} f_{n} \beta^{n},
$$

and assume that the integral $\int_{0}^{\infty} f(\beta) \mathrm{d} \beta$ exists, and that all the derivatives of $f(\beta)$ vanish faster than $1 / \beta^{1+\varepsilon}$ (with some $\varepsilon>0$ ) as $\beta \rightarrow \infty$. Then, according to Zagier [29], the small- $\beta$ asymptotics of $G(\beta)$ is given by

$$
G(\beta) \sim \frac{I_{f}}{\beta}+\sum_{n=0}^{\infty} f_{n} \zeta(-n, a) \beta^{n}
$$

with $I_{f}:=\int_{0}^{\infty} f(x) \mathrm{d} x$.

The proof goes as follows. Start with the Euler-MacLaurin formula (see Chapter 8 of $[77])$

$$
\begin{aligned}
\sum_{m=0}^{M-1} f(m+a)= & \int_{0}^{M} f(t) \mathrm{d} t+\sum_{n=0}^{N-1} \frac{B_{n+1}(a)}{(n+1) !}\left(f^{(n)}(M)-f^{(n)}(0)\right) \\
& +(-1)^{N+1} \int_{0}^{M} \frac{B_{N}(\{t-a\})}{N !} f^{(N)}(t) \mathrm{d} t .
\end{aligned}
$$

In the above equation, we have assumed $0 \leq a<1$, we have used the Bernoulli polynomials $B_{i}(x)$, and employed the fractional-part function $\{z\}=z-\lfloor z\rfloor$. 
Scaling the argument of $f(*)$ as $f(* \cdot x)$, taking the limit $M \rightarrow \infty$, and recalling that for all $n \geq 0$ we have $f^{(n)}(M) \rightarrow 0$ as $M \rightarrow \infty$, we arrive at

$$
\begin{aligned}
\sum_{m=0}^{\infty} f((m+a) x)= & \frac{1}{x} \int_{0}^{\infty} f(t) \mathrm{d} t+\sum_{n=0}^{N-1} f_{n} \zeta(-n, a) x^{n} \\
& +\left[(-1)^{N+1} \int_{0}^{\infty} \frac{B_{N}(\{t / x-a\})}{N !} f^{(N)}(t) \mathrm{d} t\right] x^{N-1} .
\end{aligned}
$$

We have used the Hurwitz zeta $\zeta(-n, a)=B_{n+1}(a) /(n+1)$ instead of the Bernoulli polynomials. Recall also that the coefficients of the asymptotic expansion of $f(x)$ around zero are given by $f_{n}=f^{(n)}(0) / n$ !.

Since $N$ can be taken to be arbitrarily large, eq. (B.5) establishes (B.3).

Armed with the above technique, we now analyze the Schur partition function of the $\mathrm{SU}(N) \mathcal{N}=4 \mathrm{SYM}$. The Schur index of this theory is observed in [7] to be proportional to the partition function of a free-fermion system on a circle. This free-fermion partition function $Z(N)$ is determined via

$$
Z(N)=\sum_{\sum_{\ell} \ell m_{\ell}=N} \prod_{\ell}(-1)^{(\ell-1) m_{\ell}} \frac{Z_{\ell}^{m_{\ell}}}{m_{\ell} ! \ell^{m_{\ell}}},
$$

in terms of the so-called spectral traces $Z_{\ell}$, given by

$$
Z_{\ell}=\sum_{p \in \mathbb{Z}}\left(\frac{1}{q^{\frac{p}{2}-\frac{1}{4}}+q^{-\frac{p}{2}+\frac{1}{4}}}\right)^{\ell} .
$$

The claim in [7] (see also [78]) is that

$$
\mathcal{I}_{\mathrm{SU}(N)}^{\mathrm{Schur}} \mathcal{N}=4(\beta)=\frac{q^{-\left(N^{2}-1\right) / 8}}{\Delta_{N}} \frac{\eta^{2}(\tau / 2)}{\eta^{4}(\tau)} Z(N),
$$

where $\Delta_{N}$ is given for odd $N$ by

$$
\Delta(N)=\frac{\vartheta_{2}}{\vartheta_{3}}=\frac{2 \eta^{4}(2 \tau) \eta^{2}(\tau / 2)}{\eta^{6}(\tau)}
$$

while for even $N$ we have $\Delta_{N}=1$. (See [7] for the definition of the functions $\vartheta_{2}, \vartheta_{3}$.)

Combining (B.8) and (4.14) we obtain

$$
Z_{\mathrm{SU}(N) \mathcal{N}=4}^{\mathrm{Schur}}(\beta)=\frac{1}{\Delta_{N}} \frac{\eta^{2}(\tau / 2)}{\eta^{4}(\tau)} Z(N)
$$

To analyze the high-temperature asymptotics of $Z_{\mathrm{SU}(N)}^{\mathrm{Schu}} \mathcal{N}=4(\beta)$ we first note that

$$
\ln \Delta(N) \sim 0 \quad(\beta \rightarrow 0),
$$

irrespective of whether $N$ is even or odd. This follows from the asymptotics of $\eta(\tau)$, and we leave its verification to the interested reader. 
Next, we rewrite the spectral traces, in a way that makes the application of Zagier's method straightforward

$$
\begin{aligned}
Z_{\ell} & =\sum_{p \geq 1}\left(\frac{1}{q^{\frac{p}{2}-\frac{1}{4}}+q^{-\frac{p}{2}+\frac{1}{4}}}\right)^{\ell}+\sum_{p \geq 0}\left(\frac{1}{q^{-\frac{p}{2}-\frac{1}{4}}+q^{\frac{p}{2}+\frac{1}{4}}}\right)^{\ell} \\
& =2 \sum_{p \geq 0}\left(\frac{1}{q^{-\frac{p}{2}-\frac{1}{4}}+q^{\frac{p}{2}+\frac{1}{4}}}\right)^{\ell} \\
& =\sum_{p \geq 0} f_{\ell}((p+1 / 2) \beta)
\end{aligned}
$$

with

$$
f_{\ell}(\beta)=2\left(\frac{1}{e^{\beta / 2}+e^{-\beta / 2}}\right)^{\ell}
$$

Applying (B.3), and using

$$
\zeta(s, 1 / 2)=\left(2^{s}-1\right) \zeta(s)
$$

we find that

$$
Z_{\ell} \sim \frac{I_{f_{\ell}}}{\beta}
$$

to all orders in $\beta$, and with

$$
I_{f_{\ell}}=2 \int_{0}^{\infty} \mathrm{d} x\left(\frac{1}{e^{x / 2}+e^{-x / 2}}\right)^{\ell}=\frac{1}{2^{\ell-2}} \frac{(\ell-2) ! !}{(\ell-1) ! !} \times \begin{cases}\frac{\pi}{2} & (\ell \text { odd }) \\ 1 & (\ell \text { even }) .\end{cases}
$$

Note that $I_{f_{1}}=\pi$.

Since $Z_{\ell}$ is (asymptotically) inversely proportional to $\beta$, the leading behavior of $Z(N)$ is found from (B.6) to be

$$
Z(N) \approx \frac{(\pi / \beta)^{N}}{N !}
$$

with an error that is down by a factor of $\beta$.

Combining (B.17), (B.11), and (B.10), we arrive at

$$
\ln Z_{\mathrm{SU}(N)}^{\mathrm{Schur}} \mathcal{N}=4(\beta)=(N-1) \ln \left(\frac{2 \pi}{\beta}\right)-(N-1) \ln 2-\ln N !+o(1),
$$

in perfect accord with (4.23). 
Note that since (B.15) is all-orders exact, we can combine it with (B.6) and (B.10) to write the all-orders asymptotic relation

$$
\ln Z_{\mathrm{SU}(N)}^{\mathrm{Schur}} \mathcal{N}=4(\beta) \sim \ln \left(P_{N-1}(\beta) / \pi\right),
$$

with $P_{N-1}(\beta)$ the degree $N-1$ polynomial in $1 / \beta$ defined by

$$
P_{N-1}(\beta)=\sum_{\sum_{\ell} \ell m_{\ell}=N}\left(\frac{1}{\beta}\right)^{\sum m_{\ell}-1} \prod_{\ell}(-1)^{(\ell-1) m_{\ell}} \frac{I_{f_{\ell}}^{m_{\ell}}}{m_{\ell} ! \ell^{m_{\ell}}} .
$$

The $\mathrm{SU}(2)$ case is easy to analyze explicitly. Since $I_{f_{1}}=\pi$ and $I_{f_{2}}=1$, we have

$$
\ln Z_{\mathrm{SU}(2)}^{\mathrm{Schur}} \mathcal{N}=4(\beta) \sim \ln \left(\frac{\pi}{2 \beta}-\frac{1}{2 \pi}\right) .
$$

Open Access. This article is distributed under the terms of the Creative Commons Attribution License (CC-BY 4.0), which permits any use, distribution and reproduction in any medium, provided the original author(s) and source are credited.

\section{References}

[1] A. Strominger and C. Vafa, Microscopic origin of the Bekenstein-Hawking entropy, Phys. Lett. B 379 (1996) 99 [hep-th/9601029] [INSPIRE].

[2] J.C. Breckenridge, R.C. Myers, A.W. Peet and C. Vafa, D-branes and spinning black holes, Phys. Lett. B 391 (1997) 93 [hep-th/9602065] [INSPIRE].

[3] P. Kraus and F. Larsen, Partition functions and elliptic genera from supergravity, JHEP 01 (2007) 002 [hep-th/0607138] [INSPIRE].

[4] C. Romelsberger, Counting chiral primaries in $\mathcal{N}=1, d=4$ superconformal field theories, Nucl. Phys. B 747 (2006) 329 [hep-th/0510060] [INSPIRE].

[5] G. Festuccia and N. Seiberg, Rigid supersymmetric theories in curved superspace, JHEP 06 (2011) 114 [arXiv:1105.0689] [INSPIRE].

[6] J. Kinney, J.M. Maldacena, S. Minwalla and S. Raju, An index for 4 dimensional super conformal theories, Commun. Math. Phys. 275 (2007) 209 [hep-th/0510251] [INSPIRE].

[7] J. Bourdier, N. Drukker and J. Felix, The exact Schur index of $\mathcal{N}=4$ SYM, JHEP 11 (2015) 210 [arXiv:1507.08659] [inSPIRE].

[8] L. Di Pietro and Z. Komargodski, Cardy formulae for SUSY theories in $d=4$ and $d=6$, JHEP 12 (2014) 031 [arXiv: 1407.6061] [INSPIRE].

[9] A.A. Ardehali, J.T. Liu and P. Szepietowski, High-Temperature Expansion of Supersymmetric Partition Functions, JHEP 07 (2015) 113 [arXiv:1502.07737] [INSPIRE].

[10] B. Assel, D. Cassani and D. Martelli, Localization on Hopf surfaces, JHEP 08 (2014) 123 [arXiv: 1405.5144] [INSPIRE].

[11] F.A. Dolan and H. Osborn, Applications of the Superconformal Index for Protected Operators and q-Hypergeometric Identities to $N=1$ Dual Theories, Nucl. Phys. B 818 (2009) 137 [arXiv:0801.4947] [INSPIRE]. 
[12] V.P. Spiridonov and G.S. Vartanov, Elliptic Hypergeometry of Supersymmetric Dualities, Commun. Math. Phys. 304 (2011) 797 [arXiv:0910.5944] [InSPIRE].

[13] E.M. Rains, Limits of elliptic hypergeometric integrals, Ramanujan J. 18 (2009) 257 [math/0607093].

[14] B. Svetitsky and L.G. Yaffe, Critical Behavior at Finite Temperature Confinement Transitions, Nucl. Phys. B 210 (1982) 423 [INSPIRE].

[15] L.G. Yaffe and B. Svetitsky, First Order Phase Transition in the SU(3) Gauge Theory at Finite Temperature, Phys. Rev. D 26 (1982) 963 [INSPIRE].

[16] K.A. Intriligator, N. Seiberg and S.H. Shenker, Proposal for a simple model of dynamical SUSY breaking, Phys. Lett. B 342 (1995) 152 [hep-ph/9410203] [INSPIRE].

[17] J.H. Brodie, P.L. Cho and K.A. Intriligator, Misleading anomaly matchings?, Phys. Lett. B 429 (1998) 319 [hep-th/9802092] [INSPIRE].

[18] A. Kapustin, B. Willett and I. Yaakov, Exact Results for Wilson Loops in Superconformal Chern-Simons Theories with Matter, JHEP 03 (2010) 089 [arXiv:0909.4559] [INSPIRE].

[19] D.L. Jafferis, The Exact Superconformal R-Symmetry Extremizes Z, JHEP 05 (2012) 159 [arXiv: 1012.3210] [INSPIRE].

[20] N. Hama, K. Hosomichi and S. Lee, SUSY gauge theories on squashed three-spheres, JHEP 05 (2011) 014 [arXiv: 1102.4716] [InSPIRE].

[21] A. Gadde, L. Rastelli, S.S. Razamat and W. Yan, Gauge Theories and Macdonald Polynomials, Commun. Math. Phys. 319 (2013) 147 [arXiv:1110.3740] [INSPIRE].

[22] M. Buican and T. Nishinaka, On the superconformal index of Argyres-Douglas theories, J. Phys. A 49 (2016) 015401 [arXiv: 1505.05884] [InSPIRE].

[23] V.P. Spiridonov and G.S. Vartanov, Elliptic hypergeometry of supersymmetric dualities II. Orthogonal groups, knots and vortices, Commun. Math. Phys. 325 (2014) 421 [arXiv: 1107.5788] [INSPIRE].

[24] E.M. Rains, Transformations of elliptic hypergeometric integrals, Ann. Math. 171 (2010) 169 [math/0309252].

[25] D. Kutasov and J. Lin, N=1 Duality and the Superconformal Index, arXiv:1402.5411 [INSPIRE].

[26] N. Bobev, M. Bullimore and H.-C. Kim, Supersymmetric Casimir Energy and the Anomaly Polynomial, JHEP 09 (2015) 142 [arXiv:1507.08553] [INSPIRE].

[27] C. Closset and I. Shamir, The $\mathcal{N}=1$ Chiral Multiplet on $T^{2} \times S^{2}$ and Supersymmetric Localization, JHEP 03 (2014) 040 [arXiv:1311.2430] [INSPIRE].

[28] B. Assel, D. Cassani, L. Di Pietro, Z. Komargodski, J. Lorenzen and D. Martelli, The Casimir Energy in Curved Space and its Supersymmetric Counterpart, JHEP 07 (2015) 043 [arXiv: 1503.05537] [INSPIRE].

[29] D. Zagier, The Mellin transform and other useful analytic techniques, appendix to Quantum Field Theory I: Basics in Mathematics and Physics. A Bridge Between Mathematicians and Physicists, E. Zeidler, Springer-Verlag (2006).

[30] F.A.H. Dolan, V.P. Spiridonov and G.S. Vartanov, From 4d superconformal indices to $3 d$ partition functions, Phys. Lett. B 704 (2011) 234 [arXiv:1104.1787] [INSPIRE]. 
[31] V.P. Spiridonov and G.S. Vartanov, Elliptic hypergeometric integrals and 't Hooft anomaly matching conditions, JHEP 06 (2012) 016 [arXiv: 1203.5677] [INSPIRE].

[32] V. Niarchos, Seiberg dualities and the 3d/4d connection, JHEP 07 (2012) 075 [arXiv: 1205.2086] [INSPIRE].

[33] A. Gadde and W. Yan, Reducing the 4d Index to the $S^{3}$ Partition Function, JHEP 12 (2012) 003 [arXiv: 1104.2592] [INSPIRE].

[34] Y. Imamura, Relation between the $4 d$ superconformal index and the $S^{3}$ partition function, JHEP 09 (2011) 133 [arXiv:1104.4482] [INSPIRE].

[35] O. Aharony, S.S. Razamat, N. Seiberg and B. Willett, $3 d$ dualities from $4 d$ dualities, JHEP 07 (2013) 149 [arXiv: 1305.3924] [INSPIRE].

[36] O. Aharony, S.S. Razamat, N. Seiberg and B. Willett, $3 d$ dualities from $4 d$ dualities for orthogonal groups, JHEP 08 (2013) 099 [arXiv: 1307.0511] [INSPIRE].

[37] A.A. Ardehali, J.T. Liu and P. Szepietowski, Central charges from the $\mathcal{N}=1$ superconformal index, Phys. Rev. Lett. 114 (2015) 091603 [arXiv:1411.5028] [INSPIRE].

[38] A.A. Ardehali, J.T. Liu and P. Szepietowski, $c-a$ from the $\mathcal{N}=1$ superconformal index, JHEP 12 (2014) 145 [arXiv: 1407.6024] [INSPIRE].

[39] A. Kapustin, B. Willett and I. Yaakov, Tests of Seiberg-like Duality in Three Dimensions, arXiv: 1012.4021 [INSPIRE].

[40] B. Willett and I. Yaakov, $\mathcal{N}=2$ Dualities and $Z$ Extremization in Three Dimensions, arXiv: 1104.0487 [INSPIRE].

[41] B.R. Safdi, I.R. Klebanov and J. Lee, A Crack in the Conformal Window, JHEP 04 (2013) 165 [arXiv: 1212.4502] [INSPIRE].

[42] D.J. Gross, R.D. Pisarski and L.G. Yaffe, $Q C D$ and Instantons at Finite Temperature, Rev. Mod. Phys. 53 (1981) 43 [inSPIRE].

[43] M. Ünsal and L.G. Yaffe, (In)validity of large $N$ orientifold equivalence, Phys. Rev. D 74 (2006) 105019 [hep-th/0608180] [INSPIRE].

[44] W. Peelaers, Higgs branch localization of $\mathcal{N}=1$ theories on $S^{3} \times S^{1}$, JHEP 08 (2014) 060 [arXiv: 1403.2711] [INSPIRE].

[45] A.A. Ardehali, High-Temperature Asymptotics of the $4 d$ Superconformal Index, Ph.D. Thesis, University of Michigan, U.S.A. (2016), to appear.

[46] F. Benini, T. Nishioka and M. Yamazaki, 4d Index to 3d Index and $2 d$ TQFT, Phys. Rev. D 86 (2012) 065015 [arXiv:1109.0283] [InSPIRE].

[47] S.S. Razamat and B. Willett, Global Properties of Supersymmetric Theories and the Lens Space, Commun. Math. Phys. 334 (2015) 661 [arXiv:1307.4381] [InSPIRE].

[48] F. Nieri and S. Pasquetti, Factorisation and holomorphic blocks in 4d, JHEP 11 (2015) 155 [arXiv: 1507.00261] [INSPIRE].

[49] Y. Imamura and S. Yokoyama, Index for three dimensional superconformal field theories with general R-charge assignments, JHEP 04 (2011) 007 [arXiv: 1101.0557] [INSPIRE].

[50] P. Pouliot, Chiral duals of nonchiral SUSY gauge theories, Phys. Lett. B 359 (1995) 108 [hep-th/9507018] [INSPIRE]. 
[51] V.P. Spiridonov and G.S. Vartanov, Superconformal indices of $\mathcal{N}=4$ SYM field theories, Lett. Math. Phys. 100 (2012) 97 [arXiv:1005.4196] [InSPIRE].

[52] A. Gadde, L. Rastelli, S.S. Razamat and W. Yan, On the Superconformal Index of $N=1$ IR Fixed Points: A Holographic Check, JHEP 03 (2011) 041 [arXiv:1011.5278] [INSPIRE].

[53] D. Kutasov and N. Seiberg, Number of degrees of freedom, density of states and tachyons in string theory and CFT, Nucl. Phys. B 358 (1991) 600 [INSPIRE].

[54] K.A. Intriligator, IR free or interacting? A proposed diagnostic, Nucl. Phys. B 730 (2005) 239 [hep-th/0509085] [INSPIRE].

[55] E. Poppitz and M. Ünsal, Chiral gauge dynamics and dynamical supersymmetry breaking, JHEP 07 (2009) 060 [arXiv:0905.0634] [INSPIRE].

[56] G.S. Vartanov, On the ISS model of dynamical SUSY breaking, Phys. Lett. B 696 (2011) 288 [arXiv: 1009.2153] [INSPIRE].

[57] K. Intriligator, Aspects of supersymmetric field theories, Int. J. Mod. Phys. A 25 (2010) 391 [INSPIRE].

[58] D. Anselmi, J. Erlich, D.Z. Freedman and A.A. Johansen, Positivity constraints on anomalies in supersymmetric gauge theories, Phys. Rev. D 57 (1998) 7570 [hep-th/9711035] [INSPIRE].

[59] A. Gadde, L. Rastelli, S.S. Razamat and W. Yan, The 4d Superconformal Index from q-deformed $2 d$ Yang-Mills, Phys. Rev. Lett. 106 (2011) 241602 [arXiv:1104.3850] [INSPIRE].

[60] J.A. Minahan and D. Nemeschansky, An $N=2$ superconformal fixed point with $E_{6}$ global symmetry, Nucl. Phys. B 482 (1996) 142 [hep-th/9608047] [INSPIRE].

[61] A. Gadde, L. Rastelli, S.S. Razamat and W. Yan, The Superconformal Index of the $E_{6}$ SCFT, JHEP 08 (2010) 107 [arXiv: 1003.4244] [INSPIRE].

[62] C. Romelsberger, Calculating the Superconformal Index and Seiberg Duality, arXiv:0707.3702 [INSPIRE].

[63] K.A. Intriligator and B. Wecht, RG fixed points and flows in SQCD with adjoints, Nucl. Phys. B 677 (2004) 223 [hep-th/0309201] [INSPIRE].

[64] E. Gerchkovitz, Constraints on the R-charges of free bound states from the Römelsberger index, JHEP 07 (2014) 071 [arXiv: 1311.0487] [INSPIRE].

[65] D. Kutasov and J. Lin, Exceptional $N=1$ Duality, arXiv:1401.4168 [INSPIRE].

[66] M. Henningson and K. Skenderis, The Holographic Weyl anomaly, JHEP 07 (1998) 023 [hep-th/9806087] [INSPIRE].

[67] S.S. Gubser, Einstein manifolds and conformal field theories, Phys. Rev. D 59 (1999) 025006 [hep-th/9807164] [INSPIRE].

[68] M. Beccaria and A.A. Tseytlin, Higher spins in AdS $S_{5}$ at one loop: vacuum energy, boundary conformal anomalies and AdS/CFT, JHEP 11 (2014) 114 [arXiv:1410.3273] [INSPIRE].

[69] R. Eager, J. Schmude and Y. Tachikawa, Superconformal Indices, Sasaki-Einstein Manifolds and Cyclic Homologies, Adv. Theor. Math. Phys. 18 (2014) 129 [arXiv:1207.0573] [INSPIRE].

[70] P. Agarwal, A. Amariti and A. Mariotti, A Zig-Zag Index, arXiv:1304.6733 [INSPIRE].

[71] I. Biswas, D. Gaiotto, S. Lahiri and S. Minwalla, Supersymmetric states of $N=4$ Yang-Mills from giant gravitons, JHEP 12 (2007) 006 [hep-th/0606087] [INSPIRE]. 
[72] E. Shaghoulian, Modular forms and a generalized Cardy formula in higher dimensions, arXiv: 1508.02728 [INSPIRE].

[73] N. Seiberg, Notes on theories with 16 supercharges, Nucl. Phys. Proc. Suppl. 67 (1998) 158 [hep-th/9705117] [INSPIRE].

[74] L.D. Faddeev, R.M. Kashaev and A. Yu. Volkov, Strongly coupled quantum discrete Liouville theory. 1. Algebraic approach and duality, Commun. Math. Phys. 219 (2001) 199 [hep-th/0006156] [INSPIRE].

[75] G. Felder and A. Varchenko, The elliptic gamma function and $\operatorname{SL}(3, Z) \times Z^{3}$, Adv. Math. 156 (2000) 44 [math/9907061].

[76] A. Narukawa, The modular properties and the integral representations of the multiple elliptic gamma functions, Adv. Math. 189 (2004) 247 [math/0306164].

[77] F.W.J. Olver, Asymptotics and special functions, Academic Press, New York-London (1974).

[78] J. Bourdier, N. Drukker and J. Felix, The $\mathcal{N}=2$ Schur index from free fermions, JHEP 01 (2016) 167 [arXiv : 1510.07041] [INSPIRE]. 